\title{
WestVirginiaUniversity
}

THE RESEARCH REPOSITORY @ WVU

Graduate Theses, Dissertations, and Problem Reports

2019

\section{Enhancing Water Stress Tolerance in Floriculture Crops}

\author{
Suejin Park \\ West Virginia University, separk@mix.wvu.edu
}

Follow this and additional works at: https://researchrepository.wvu.edu/etd

Part of the Bioinformatics Commons, Biotechnology Commons, Horticulture Commons, Molecular

Genetics Commons, and the Plant Biology Commons

\section{Recommended Citation}

Park, Suejin, "Enhancing Water Stress Tolerance in Floriculture Crops" (2019). Graduate Theses, Dissertations, and Problem Reports. 7390.

https://researchrepository.wvu.edu/etd/7390

This Dissertation is protected by copyright and/or related rights. It has been brought to you by the The Research Repository @ WVU with permission from the rights-holder(s). You are free to use this Dissertation in any way that is permitted by the copyright and related rights legislation that applies to your use. For other uses you must obtain permission from the rights-holder(s) directly, unless additional rights are indicated by a Creative Commons license in the record and/ or on the work itself. This Dissertation has been accepted for inclusion in WVU Graduate Theses, Dissertations, and Problem Reports collection by an authorized administrator of The Research Repository @ WVU.

For more information, please contact researchrepository@mail.wvu.edu. 


\title{
Enhancing Water Stress Tolerance in Floriculture Crops
}

\author{
Suejin Park \\ A doctoral dissertation submitted to the \\ Davis College of Agriculture, Natural Resources and Design \\ at West Virginia University \\ in partial fulfillment of the requirements for the degree of \\ Doctor of Philosophy \\ in \\ Plant and Soil Sciences
}

\author{
Nicole Waterland, Ph.D., Chair \\ Vagner Benedito, Ph.D. \\ Jennifer Hawkins, Ph.D. \\ Youyoun Moon, Ph.D. \\ Sven Verlinden, Ph.D. \\ Jianbo Yao, Ph.D. \\ Division of Plant and Soil Sciences
}

Morgantown, West Virginia

2019

Keywords: Floriculture, water deficit stress, antitranspirant, osmotic treatment, RNAseq Copyright 2019 Suejin Park 


\section{ABSTRACT \\ Enhancing Water Stress Tolerance in Floriculture Crops}

Suejin Park

Water deficit is one of the major constraints on plant growth and development, causing reduction of crop productivity. To minimize water loss, among many adaptation strategies, plants close their stomata to reduce transpiration. The stomatal closure is regulated by light, internal $\mathrm{CO}_{2}$ concentration, and plant hormones, mainly abscisic acid. Plants' response mechanisms to water deficit are complex processes involving numerous genes and various signaling pathways. Floriculture crops are often exposed to water deficit during shipping and retailing, and these periods often result in damaged crops and profit loss. Understanding of plant responses to water deficit stress will provide us an opportunity to develop floriculture crops with enhanced water deficit tolerance.

The long-term goal of this research is to enhance water deficit stress tolerance in floriculture crops employing physiological and genetic technologies. As a practical approach, five antitranspirants, chemicals to reduce transpirational water loss, were evaluated for enhancing temporary water deficit tolerance in bedding plants. One physical $[\beta$-pinene polymer $(\beta \mathrm{P})]$ and one physiological [a biological active form of abscisic acid (s-ABA)] antitranspirants enhanced tolerance to water deficit stress by blocking stomatal and inducing stomatal closure, respectively. However, $\beta \mathrm{P}$ caused floral damage and s-ABA caused chlorosis on the margin of leaves in some cultivars. To develop a more effective tool with minimal damage to plants, osmotic treatments was examined in viola. The osmotic treatment with high concentration of $\mathrm{CaCl}_{2}$ induced stomatal closure, resulting in delayed plant wilting through reduction of transpirational water loss and maintenance of high water content in plant leaves. In addition, $\mathrm{CaCl}_{2}$ treatment did not show any visual damage on plants. The osmotic treatment will allow floriculture crops to temporary tolerate water deficit stress without significant loss of postproduction quality.

To investigate mechanisms of water stress responses at the molecular level, time-course transcriptome analysis was performed in petunia leaves at the early stage of water deficit. A total 6670 genes were differentially expressed under water deficit stress conditions. Gene Ontology analysis revealed that redox homeostasis processes with sulfur metabolism were enriched under water deficit stress. Genes encoding components of plant hormones, abscisic acid and ethylene, biosynthesis and signal transduction pathways were differentially expressed at the early stage of water deficit. Thirty-four transcription factor families were identified in petunia, and one member of AP2/ERF family, PhERF039, were selected for functional analysis. Underexpression of $P h E R F 039$ reduced stomatal conductance in addition to phenotypes related to development: delayed flowering and development of a rosette morphology. Although the result of stomatal conductance seemed to indicate that $P h E R F 039$ might play as a negative regulator in stomatal closure under water deficit, further analysis is required using additional independent transgenic lines. Transcriptomic data may provide insights into understanding water stressresponsive networks as well as opportunities to engineer floricultural crops with the enhanced water deficit tolerance traits. 


\section{DEDICATION}

I would like to dedicate my dissertation to my parents,

$$
\begin{gathered}
\text { 박은우 and 길근미, and } \\
\text { to my brother's family, } \\
\text { 박근환, 김혜란, 박준영, and 박시영. }
\end{gathered}
$$

I am so lucky to have you and I love you so much. 


\section{ACKNOWLEDGMENTS}

I would like to thank my advisor, Dr. Nicole Waterland, for her guidance, encouragement and support. Also, I sincerely thank Dr. Youyoun Moon for his advice, training, and motivation. I have learned what kind of mind and philosophy I should have as a good scientist from them. I could not have finished my dissertation successfully without their support, patience, and inspiration. I will never forget their passion, kindness, and love.

I would also like to thank my committee members, Dr. Vagner Benedito, Dr. Jennifer Hawkins, Dr. Sven Verlinden, and Dr. Jianbo Yao for their advice, guidance, and feedback on my research.

I especially thank Sarah Mills, my first friend in Morgantown and now my best friend in life. She is a wonderful friend, colleague, English teacher, translator, and life mentor. I cannot thank her enough for her help and patience.

I would like to acknowledge the Division of Plant and Soil Sciences and the West Virginia University Evansdale Greenhouse Staff for supporting my research.

Finally, I would like to thank my family and friends for their encouragement and support. Specially, I thank my parents, Eun Woo Park and Keunmi Kil, my brother, Keunhwan Park, and his family for their love, support, and patience. I love them so much. 


\section{TABLE OF CONTENTS}

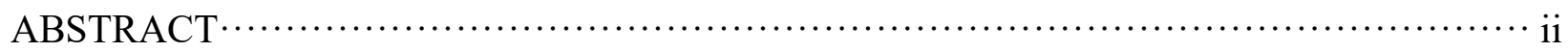

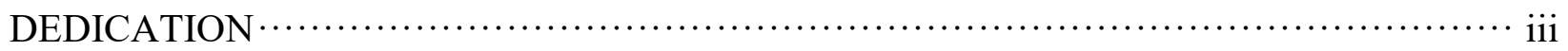

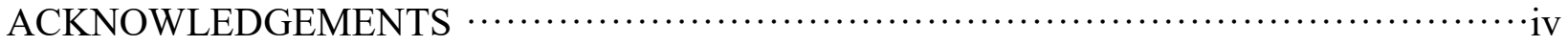

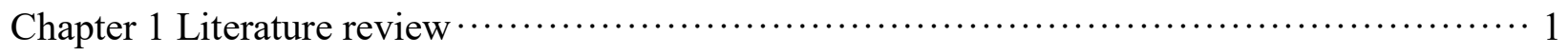

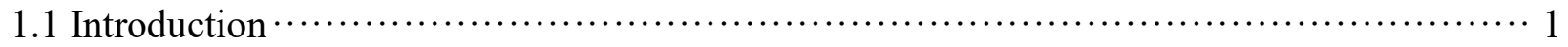

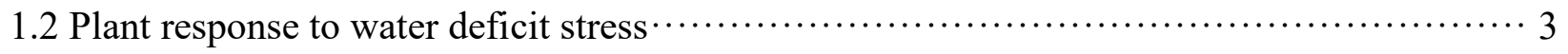

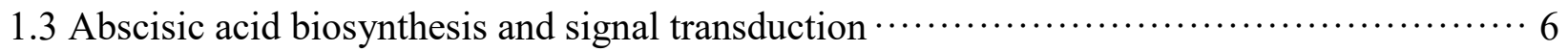

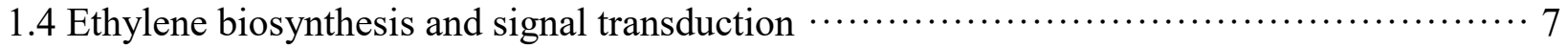

1.5 Practical and genetic approaches to enhance water deficit tolerance $\cdots \cdots \cdots \cdots \cdots \cdots \cdots \cdots \cdots \cdots \cdots$

Chapter 2 Evaluation of antitranspirants for enhancing temporary water stress tolerance in bedding

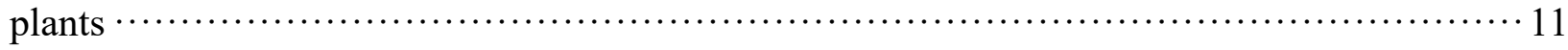

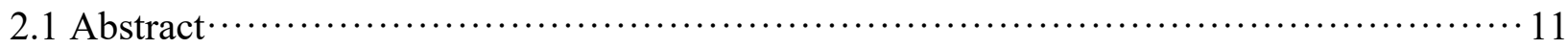

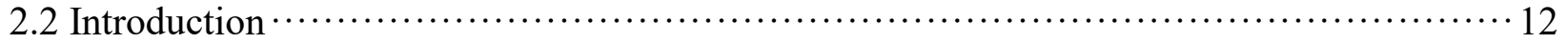

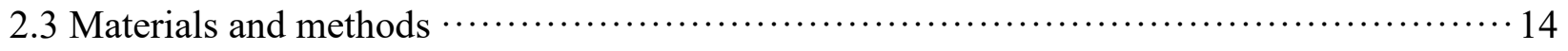

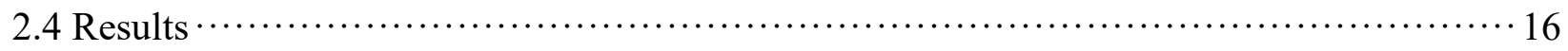

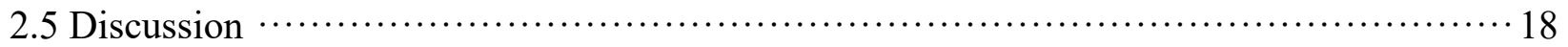

Chapter 3 Treatment of calcium chloride enhances water deficit tolerance in viola (Viola cornuta)

.

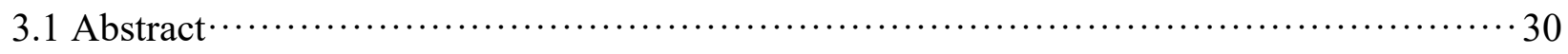

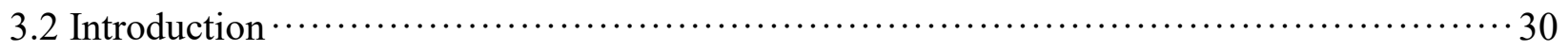

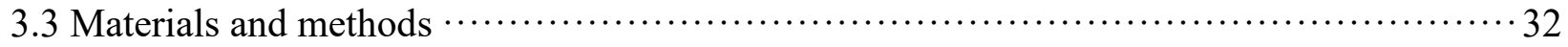

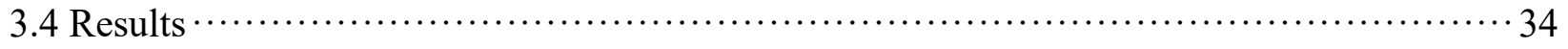

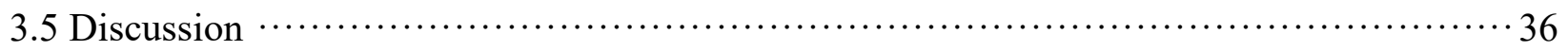

Chapter 4 Time-course transcriptomic analysis of Petunia $\times$ hybrida leaves under water deficit

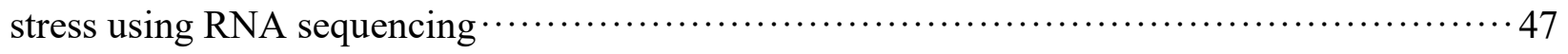

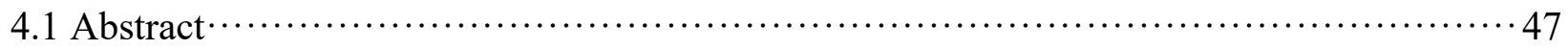

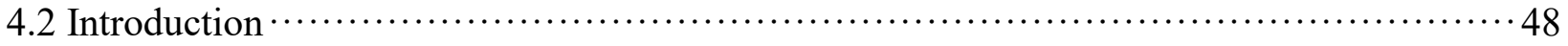

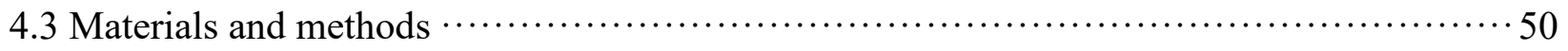




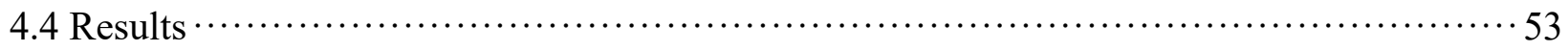

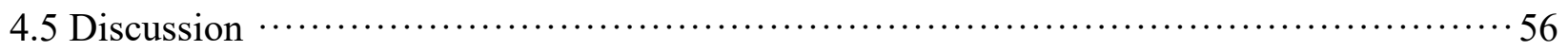

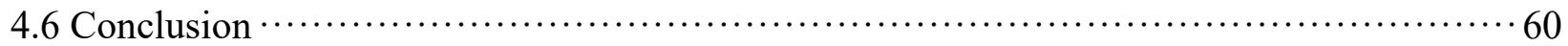

Chapter 5 Functional analysis of transcription factor PhERF039 for enhancing water stress

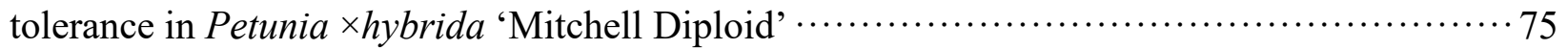

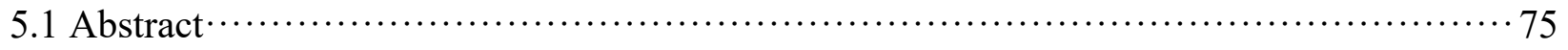

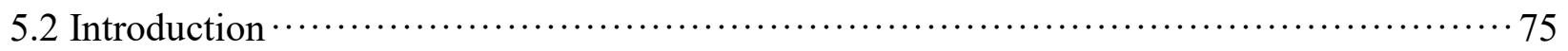

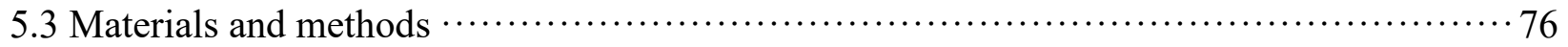

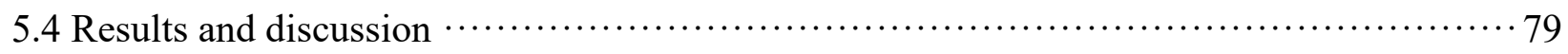

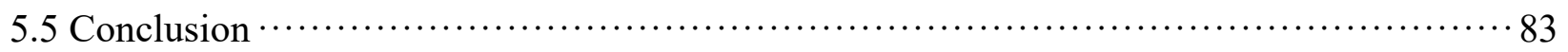

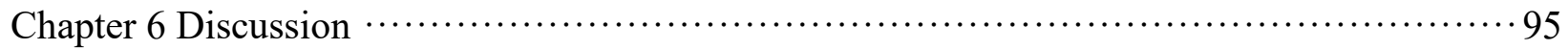

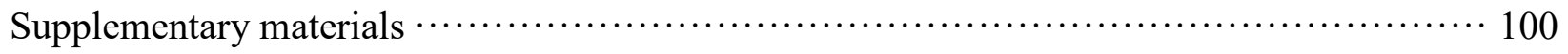

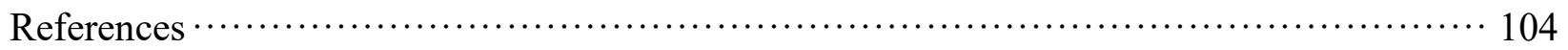




\section{LIST OF TABLES}

Table 2.1. Manufacturer's recommended application rate of antitranspirants

Table 2.2. The number of days until the appearance of wilting symptom in eight cultivars of bedding plants treated with antitranspirants at manufacturer's recommended rate (1X) under

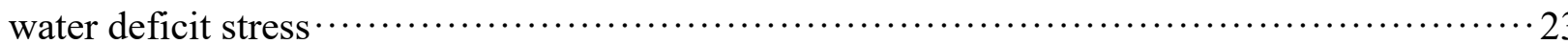

Table 2.3. Shelf life extension of two cultivars of african marigold treated with $\beta$-pinene polymer $(\beta \mathrm{P})$, and impatiens and petunia treated with a biologically active form of abscisic acid (s-ABA) at three rates under water deficit stress

Table 3.1. Shelf life, percentage of damaged plants, and necrotic area percentage (NAP) of viola

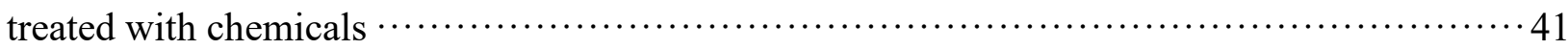

Table 3.2. Shelf life of $\mathrm{CaCl}_{2}$-treated viola under water deficit

Table 4.1. Summary of assembled contigs by Trinity and clustered transcripts with $90 \%$ similarity

Table 4.2. Biological process terms enriched commonly at least two days by upregulated genes

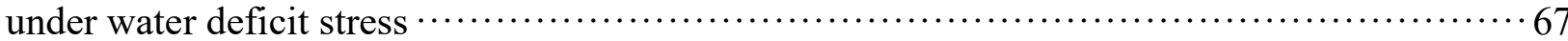

Table 4.3. Biological process terms enriched commonly on day 3 and 5 by downregulated genes

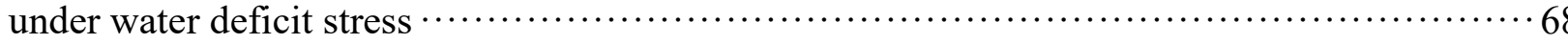




\section{LIST OF FIGURES}

Fig. 2.1. Stomatal conductance and wilt status rating of $\beta$-pinene polymer $(\beta P)$-treated 'Harmony Spicy Peach’ new guinea impatiens (A), ‘Antigua Yellow' african marigold (B), 'Taishan

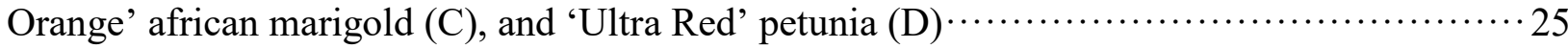

Fig. 2.2. Stomatal conductance and wilt status rating of a biologically active form of abscisic acid (s-ABA)-treated 'Harmony Spicy Peach' new guinea impatiens (A), 'Antigua Yellow' african marigold (B), 'Taishan Orange' african marigold (C), and 'Ultra Red' petunia (D) $\cdots \cdots 27$

Fig. 2.3. Floral damage of bedding plants treated with $\beta$-pinene polymer $(\beta \mathrm{P})$ at $1 \mathrm{X} \cdots \cdots \cdots \cdots \cdot \cdots$

Fig. 3.1. Osmotic potential of chemical solutions measured with a dewpoint potentiometer $\cdots \cdot 40$

Fig. 3.2. Foliage damage of viola treated by $\mathrm{NaCl}$ at $450 \mathrm{mM}$ (A), $\mathrm{NaNO}_{3}$ at $450 \mathrm{mM}$ (B), $\mathrm{Ba}\left(\mathrm{NO}_{3}\right)_{2}$ at $300 \mathrm{mM}(\mathrm{C})$, and mannitol at $730 \mathrm{mM}(\mathrm{D})$ with daily irrigation

Fig. 3.3 Daily wilt status rating of viola treated with $\mathrm{CaCl}_{2}$ at 200 and $300 \mathrm{mM}$ under water deficit stress

Fig. 3.4. Viola treated with control, $\mathrm{CaCl}_{2}$ at 200 and $300 \mathrm{mM}$ (from left to right)

Fig. 3.5. Stomatal conductance ( $g \mathrm{~s}, \mathrm{~A}$ and $\mathrm{B}$ ) and leaf relative water content $(\mathrm{RWC}, \mathrm{C}$ and $\mathrm{D})$ of

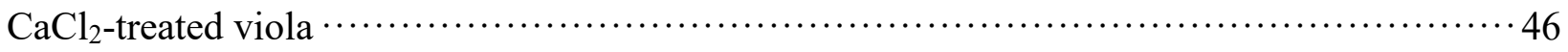

Fig. 4.1. Stomatal conductance of control and water-stressed plants for five days after withholding water

Fig. 4.2. Histogram of Gene Ontology (GO) classification of all transcripts into three main categories including cellular component, molecular function, and biological process $\cdots \cdots \cdots \cdots \cdot 64$

Fig. 4.3. Hierarchical clustering analysis of differentially expressed genes (DEGs) $\cdots \cdots \cdots \cdots \cdots \cdots$

Fig. 4.4. Number of differentially expressed genes (DEGs) under water deficit stress $\cdots \cdots \cdots \cdot \cdots 6$

Fig. 4.5. Heatmap of differentially expressed genes involved in abscisic acid and ethylene biosynthesis and signal transduction pathways under water deficit stress $\ldots \ldots \ldots \ldots \ldots \ldots \ldots \ldots \ldots \ldots$

Fig. 4.6. Abscisic acid and ethylene signal transduction Kyoto Encyclopedia of Genes and Genomes pathway 
Fig. 4.7. Distribution of differentially expressed transcription factor (TF) families

Fig. 4.8. Consistency between RNA-seq data and qPCR data

Fig. 4.9. Heatmap of differentially expressed genes involved in redox homeostasis processes under water deficit stress

Fig. 4.10. Fold change in gene expression of AP2/ERF TFs under water deficit stress compared to control

Fig. 5.1. Comparison of deduced amino acid sequences of ERF family proteins that have high

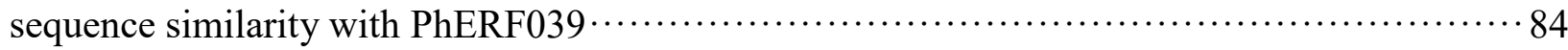

Fig. 5.2. Expression levels of PhERF039 under water deficit stress $\cdots \cdots \cdots \cdots \cdots \cdots \cdots \cdots \cdots \cdots \cdots \cdots \cdots \cdots \cdots \cdots \cdots \cdots$

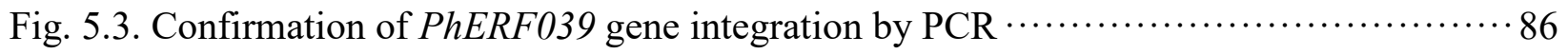

Fig. 5.4. Expression levels of PhERF039 in wild type (WT) and one transgenic line $\mathrm{T}_{0}(\mathrm{~A})$ and

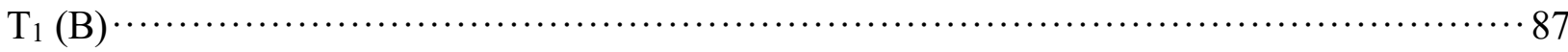

Fig. 5.5. Plant growth and development of wild type (WT) and the transgenic PhERF039 (UE;

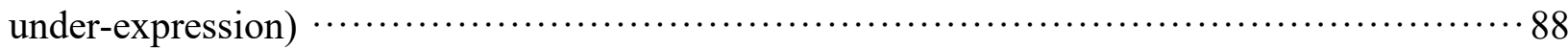

Fig. 5.6. Flower development of wild type (WT) and the transgenic PhERF039 (UE; underexpression)

Fig. 5.7. Daily average substrate volumetric water content throughout the water deficit period for wild type (WT, A) and the transgenic PhERF039 (UE; under-expression, B) …….............. 90

Fig. 5.8. Wilt status rating of wild type (WT) and the transgenic PhERF039 (UE; underexpression) at various volumetric water contents: 0.25 (A), 0.15 (B), 0.10 (C), and 0.05 (D) $\mathrm{m}^{3} \cdot \mathrm{m}^{-3}$

Fig. 5.9. Stomatal conductance (gs) of wild type (WT) and the transgenic PhERF039 (UE; under-expression) at various volumetric water contents: 0.25 (A), 0.15 (B), 0.10 (C), and 0.05 (D) $\mathrm{m}^{3} \cdot \mathrm{m}^{-3}$ 
Fig. 5.10. Relationship between volumetric water content in the substrate and stomatal conductance in wild type (WT) and the transgenic PhERF039 (UE; under-expression) under

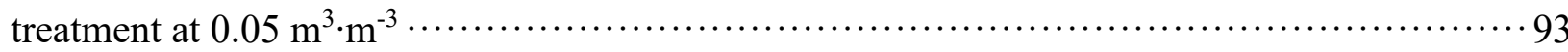

Fig. 5.11. Relationship between volumetric water content and stomatal conductance in wild type (WT) and the transgenic PhERF039 (UE; under-expression) under volumetric water content at

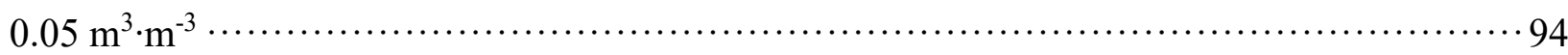




\section{Chapter 1 Literature review}

\subsection{Introduction}

Floriculture crops are often prone to damage after harvest (e.g., during shipping and retailing), although they are produced to the highest quality (Ferrante et al., 2015). In fact, these postproduction conditions are usually characterized by inadequate environmental conditions for

plants, such as high temperatures and irregular irrigation, consequently causing water deficit stress and plant wilting (Ferrante et al., 2015). It is estimated that crop loss due to water deficit is up to $20 \%$ of unsalable crops (Healy, 2009). Water plays a crucial role in plant growth and development and is indispensable for proper function of the cell. Water is the most abundant and fundamental solvent, where most biochemical reactions occur, and it is the vital reagent of many chemical reactions in plants, such as photosynthesis and cellular respiration. Also, water generates turgor pressure, the force per unit area of a liquid pushing the plasma membrane against the cell wall. Turgor pressure is important for cell enlargement, transport processes across membranes, and the rigidity and mechanical stability of plant tissues (Taiz and Zeiger, 2010).

Water is taken up from roots and transpired through stomata to the atmosphere. While water escapes from leaves, heat is dissipated to prevent the leaf surface temperature from rising. At the same time, minerals are transported from roots to leaves through the soil-plant-atmosphere continuum, providing essential nutrients for plants (Taiz and Zeiger, 2010). However, water availability is often limited for plants, and it is one of the major environmental constraints to plant's growth and development. When the water supplied to plants is inadequate, it could lead to severe crop loss. Therefore, it is strongly desired not only to understand the plants' response mechanism to water deficit stress, but also to develop water stress tolerant crops and invent new technologies to minimize crop damage caused by water deficiency.

Water deficit tolerance can be enhanced by exogenous application of hormones and other chemicals (Farooq et al., 2009). One of the short-term and most practical approaches to overcome the adverse effects of water stress is application of phytohormone. Exogenous application of a stress-hormone, abscisic acid (ABA), has proven to enhance temporary water stress tolerance in various plants by limiting transpirational water loss (Waterland et al., 2010b). Applications of osmoprotectants, such as polyamines, proline, and glycine betaine, have been reported to enhance plants' ability to tolerate water deficit stress by mediating osmotic 
adjustment and protecting subcellular structures under water stress (Bhargava and Sawant, 2013).

It is essential to understand the molecular mechanisms of water deficit response in order to develop crops with enhanced tolerance. Plants perceive stress signals by the receptors on the plasma membrane of plant cells (Tuteja and Sopory, 2008). Signal perception is followed by signal transduction that activates a large number of stress-related genes and synthesizes functional proteins that ultimately lead to various physiological and metabolic responses for survival under water-deficient conditions (Tuteja and Sopory, 2008). The expression and activity of these functional proteins are controlled by regulatory proteins, such as proteins involved in signal transduction and transcription factors (TFs). Manipulation of regulatory genes has become a powerful tool for improving water stress tolerance because they function as master switches that regulate the response mechanisms to water stress (Bhargava and Sawant, 2013; BhatnagarMathur et al., 2008).

Despite significant efforts during the past decades in aiming to elucidate mechanisms of water stress responses, the comprehensive pathways of water stress tolerance have not been fully understood. Also, most studies identifying pathways have been focused on model plants such as Arabidopsis and food crops: rice and corn. There has been relatively little documented research regarding water stress with floriculture crops.

The goal of this research was to enhance water deficit tolerance of floriculture crops by employing physiological and genetic technologies. To achieve this goal, understanding the physiological and molecular mechanisms of water stress response is essential. In addition, the development of practical technologies based on physiological and metabolic responses to water deficit stress are also attempted to minimize floriculture crop damage.

The four specific objectives are:

1. To evaluate commercial antitranspirants for enhancing temporary water deficit tolerance in bedding plants

2. To examine the effect of osmotic treatment as a new antitranspirant to enhance water deficit tolerance in viola

3. To perform RNA-seq and analyze transcriptomic data under water deficit in petunia

4. To conduct functional analysis of PhERF039 in petunia under water deficit stress 


\subsection{Plant response to water deficit stress}

Water deficit is one of the abiotic factors limiting crop yield, and global climate change is known to cause severe drought, ultimately threatening global food security (Basu et al., 2016; Farooq et al., 2009). Severe water deficiency causes decline in crop productivity by negatively affecting plant growth and reproduction (Fahad et al., 2017). Plants exposed to water deficit often show physiological and biochemical dysfunctions (Xu et al., 2013a). Water is a vital means to facilitate mineral uptake by the roots. Under water deficit condition, retarded growth often occurs due to reduced mineral uptake. Another key physiological dysfunction is reduced photosynthesis. When water is not sufficiently supplied to plants, stomata are closed to minimize water loss, but it also reduces $\mathrm{CO}_{2}$ uptake. Water deficit stress negatively affects plants growth and development through reduced leaf expansion, impaired photosynthetic machinery, and reduced activities of enzymes involved in photosynthesis (Fahad et al., 2017). Exposure to water deficit also causes oxidative damage by the formation of reactive oxygen species (ROS). The level of ROS increases due to the imbalance between the generation and utilization of electrons. Reactive oxygen species leads severe cellular damage by peroxidation of membrane lipid and degradation of enzymes and nucleic acids (Farooq et al., 2009).

To survive and sustain growth under water deficiency, plants must acclimate to the environment by initiating various responses and adaptive mechanisms (Fang and Xiong, 2015). These adaptive traits include the optimization of water uptake and minimization of water loss. Under water deficit stress, plants develop extensive root systems and reach deeper places in soil in order to increase water uptake. Furthermore, plants reduce transpirational water loss by inducing stomata closure, leaf folding, and cuticle wax synthesis in order to use water more efficiently (Basu et al., 2016; Lamaoui et al., 2018). Plants also endure water deficit stress by maintaining cell turgor pressure with high accumulation of osmolytes such as sugars, proline, and polyamines (Farooq et al., 2009). Other important defense mechanism, an antioxidant system, operates against a surge of ROS by increasing the activities of oxidation-protective enzymes such as superoxide dismutase, ascorbate peroxidase, catalase, and glutathione reductase (Fang and Xiong, 2015). Stabilizing cell membranes is also a crucial mechanism to survive under water stress (Farooq et al., 2009).

With a help of the advanced molecular tools, a number of genetic and molecular mechanisms of water stress tolerance has been unveiled in last decade (Bhargava and Sawant, 2013). Recent 
findings suggest that plants' response mechanism to water stress is a complex process involving numerous genes and various signaling pathways (Fang and Xiong, 2015). External stimuli are first perceived by the receptors on the membrane of the root cells, and secondary messengers (e.g., ABA, $\mathrm{Ca}^{2+}$, nitric oxide, and ROS) contribute to signal transduction (Bhargava and Sawant, 2013). Then, phosphorylation cascades are initiated and/or water stress-response genes are expressed, consequently physiological and morphological changes take place (Obidiegwu et al., 2015). Genes involved in water deficit stress response are mainly classified into two groups: (1) the regulatory genes encoding kinases and phosphatases, and TFs that regulate signal transduction pathways and transcription of downstream target genes, and (2) the functional genes that are directly involved in protecting plants against potential damage caused by water stress, e.g., genes encoding aquaporins and ion transporters, and enzymes producing antioxidants (Hadiarto and Tran, 2011). Secondary messengers and regulatory genes have been implicated as key regulators in plant adaptation to water stress (Fang and Xiong, 2015).

Various secondary messengers have been known to modulate the expression and activity of TFs and functional genes (Tuteja and Mahajan, 2007). These molecules include ABA, $\mathrm{Ca}^{2+}$, nitric oxide, ROS, and sugars, and among them $\mathrm{ABA}$ and $\mathrm{Ca}^{2+}$ represent the most essential secondary messengers. Abiotic stresses such as water deficit stress induce ABA synthesis; hence it is regarded as a plant stress hormone (Tuteja and Sopory, 2008). Several studies have reported that $\mathrm{ABA}$ causes a rapid increase in cytosolic free calcium level, which initiates the stress signal transduction pathways. The elevated concentration of cytosolic calcium is sensed by various calcium-binding proteins such as calmodulin (CaM), CaM-binding proteins, and calciumdependent protein kinases (CDPKs or CPKs in Arabidopsis). These proteins can trigger the expression of downstream gene(s) by initiating a phosphorylation cascade, which can elicit specific responses to water stress (Tuteja and Sopory, 2008).

Stress-induced stomatal closure is a well-known effect of ABA and calcium. Onset of water stress triggers ABA synthesis in the root and ABA is transported to the leaves by the xylem sap, and it reaches ABA receptors on the membrane of the guard cells. ABA-bound receptors activate $\mathrm{Ca}^{2+}$ channels in the plasma membrane and induce $\mathrm{Ca}^{2+}$ release from vacuole, and as a result, the concentration of cytosolic calcium in guard cells increases (Tuteja and Mahajan, 2007). The elevated level of calcium activates CPKs to open two types of anion channels: slow (S-type) and rapid (R-type)(Kuromori et al., 2014; Roelfsema et al., 2012). The prolonged activation of these 
anion channels allows anion efflux, leading to depolarization of the plasma membrane. Consequently, this status causes $\mathrm{K}^{+}$to move out of guard cells via outward-rectifying potassium channels (Roelfsema et al., 2012). The efflux of ions (anions and $\mathrm{K}^{+}$) changes osmotic potential that drives reduction of turgor pressure of guard cells, resulting in stomatal closure. Furthermore, ABA-induced calcium prevents light-induced stomatal opening by inactivation of $\mathrm{H}^{+}$-ATPase and inward $\mathrm{K}^{+}$channels (Brault et al., 2004; Kinoshita et al., 1995). As a result of inducing stomatal closure and preventing stomatal opening, plants can reduce transpirational water loss and withstand against water deficit stress.

$\mathrm{ABA}$ also acts as a signal to regulate the expression of genes encoding a wide range of proteins and enzymes involved in dehydration responses (Yates et al., 2014). Most of ABAinducible genes are regulated by a cis-acting element, called ABA responsive element (ABRE), in their promoter regions. ABRE is targeted by the corresponding TFs, ABRE-binding proteins/factors (AREBs/ABFs) which belong to basic leucine zipper (bZIP) family. AREB/ABF TFs are mainly expressed in vegetative tissues under water deficit condition, and their gene activity is also regulated by ABA-dependent phosphorylation. The phosphorylation of AREB/ABF is catalyzed by ABA-activated SNF1-related protein kinase 2 (SnRK2). Therefore, ABA is required to induce expression of genes that contain the cis-acting element, ABRE. In addition to ABA-dependent pathways, there is an ABA-independent pathway. A number of genes responding to water stress were induced in a mutant defective in ABA biosynthesis or signaling, revealing ABA-independent pathways (Kuromori et al., 2014). The promoter region of these genes has a cis-element, drought-response element/C-repeat (DRE/CRT). Major TFs that recognize DRE/CRT are DRE-binding protein 1/CRT-binding factor (DREB1/CBF, cold stress) and DREB2 (water deficit stress) which belong to APETALA2/ethylene-responsive elementbinding factor (AP2/ERF) family (Kuromori et al., 2014). In plants, both ABA-dependent and ABA-independent pathway play important roles in the transcriptional regulatory networks under water stress.

It is increasingly becoming evident that another phytohormone, ethylene, can have direct and/or indirect effects on stress responses in plants (Kazan, 2015). Ethylene is synthesized in response to both abiotic and biotic stresses, suggesting it regulates plant adaptations against adverse environmental changes (Dubois et al., 2018). In contrast to ABA which induce stomatal closure, ethylene has been reported to be involved in both stomatal opening and closure. 
Ethylene inhibited ABA-induced stomatal closure and an ethylene-overproducing mutant (etol1) delayed stomatal closure in water deficit condition (Tanaka et al., 2005). ABA-induced stomatal closure was also inhibited by an application of the ethylene precursor, 1aminocyclopropane-1-carboxylic acid (ACC)(Tanaka et al., 2006). These results indicated that the negative effect of ethylene on ABA-induced stomatal closure under water deficit stress. Ethylene also reduced ROS production, which is another secondary messenger to increase cytosolic calcium level in guard cells and active anion efflux channels, ultimately induce stomatal closure under water deficit (Singh et al., 2017). Watkins et al. (2014) suggested that ethylene increased flavanols which scavenge ROS and inhibited stomatal closure. However, ethylene also induced stomatal closure by producing $\mathrm{H}_{2} \mathrm{O}_{2}$ through NADPH oxidase (Desikan et al., 2006). It is still unclear how plants regulate stomatal opening and closing in response to ethylene under water deficit.

Ethylene is also responsible for transcriptional regulation of genes related to ethylene and abiotic stresses by activating TFs, EIN3/EIL1 (Tao et al., 2015). These TFs bind to the promoter of ethylene-responsive factors (ERFs) and activate expression of $E R F s$. Ethylene-response factors are considered as important TFs. They belong to the plant-specific AP2/ERF TF family which play important roles in plant adaptation under abiotic stresses (Xie et al., 2019). For example, the expression of AtERF1 was highly induced under salinity and water deficit stress, and the overexpressing of $A t E R F 1$ enhanced tolerance to salt, water deficit, and heat stresses in Arabidopsis (Cheng et al., 2013). A tomato ERF protein TSRF1 improved the osmotic and drought tolerance in rice seedling by increasing osmoprotectants (prolines and soluble sugars)(Quan et al., 2010). Therefore, ERFs have been highlighted as key regulators of abiotic stress responses (Debbarma et al., 2019)

\subsection{Abscisic acid biosynthesis and signal transduction}

Abscisic acid is a phytohormone associated with leaf abscission, bud dormancy, seed dormancy, suppression of elongation and stomatal closure (Taiz and Zeiger, 2010). The level of $\mathrm{ABA}$ in plants increases during abiotic stress, and the elevated $\mathrm{ABA}$ can lead to plant adaptation to various abiotic stresses (Sah et al., 2016).

ABA is a sesquiterpenoid $\left(\mathrm{C}_{15}\right)$ synthesized from carotenoid $\left(\mathrm{C}_{40}\right)$, zeaxanthin (Taiz and Zeiger, 2010). The carotenoid is cleaved by 9-cis-epoxycarotenoid dioxygenase (NCED), 
producing the intermediate xanthoxin $\left(\mathrm{C}_{15}\right)$ in the plastid (Sah et al., 2016). Then xanthoxin moves into the cytosol, where xanthoxin is converted to ABA by two enzymatic reactions. Xanthoxin is modified into abscisic aldehyde by an enzyme short-chain alcohol dehydrogenase/reductase (SDR) encoded by $A B A 2$. Abscisic aldehyde is oxidized into ABA by an abscisic aldehyde oxidase (AAO). Further oxidation by ABA 8'-hydroxylase (CYP707A) leads to ABA degradation. ABA can also be inactivated by conjugation with glucose (Hauser et al., 2017). Under water deficit stress, the increased levels of ABA have been reported in many plant species including wheat, rice, maize, sorghum, and soybean (Xiong and Zhu, 2003). The cleavage of the carotenoid to xanthoxin is a rate-limiting step, and thus expression of $N C E D$ has been considered as a key enzyme in the ABA biosynthesis (Vishwakarma et al., 2017). Water deficit treatments have shown to increase NCED expression in Arabidopsis (Iuchi et al., 2001), tomato (Burbidge et al., 1999), and bean (Qin and Zeevaart, 1999). A significant rise in NCED transcript levels following dehydration provides an evidence for the importance of $N C E D$ genes in ABA-mediated stress responses (Xiong and Zhu, 2003).

There are three core components of ABA signaling including ABA receptors (PYR/PYL/RCAR), protein phosphatase 2C (PP2C, negative regulators), and SNF1-related protein kinases 2 (SnRK2, positive regulators)(Sah et al., 2016). In the absence of ABA, PP2Cs inactivate SnRK2 by dephosphorylating the activation loop of SnRK2. In the presence of ABA, PYR/PYL/RCAR receptors bind to ABA, forming a PYLs-ABA-PP2c complex and releasing SnRK2. The SnRK2s are then activated by autophosphorylation of their activation loop, and they can phosphorylate downstream proteins, such as TFs, ion channels, enzymes, and activate ABAdependent pathway. In addition to AREB/ABF bZIP TFs, other TFs have been known to be involved in ABA-mediate gene expression. These TFs include several families, AP2/ERF, MYB, bHLH, C2H2-ZF, WRKY, and NAC (Fujita et al., 2011).

\subsection{Ethylene biosynthesis and signal transduction}

Ethylene is the gaseous plant hormone playing huge roles in plant growth and development (Taiz and Zeiger, 2010). Ethylene regulates many aspects of plant life, including seed germination, seedling growth, organ development and senescence, leaf and flower abscission, fruit ripening, and responses to stresses (Taiz and Zeiger, 2010). The production of ethylene has 
been known to be regulated by internal signals and external stimuli such as abiotic and biotic stresses (Kazan, 2015).

Methionine is a precursor of ethylene in plants, and most methionine is converted to Sadenosylmethionine (SAM) by SAM synthetase (SAMS)(Wang et al., 2002). Then, the first committing step in ethylene biosynthesis is the conversion of SAM into 1-aminocyclopropane-1carboxylic acid (ACC) by ACC synthase (ACS). In addition to ACC, ACS also produce 5'methylthioadenosine (MTA) which is a substrate in the recycling pathway of methionine called methionine cycle or Yang cycle. Methionine is regenerated through this salvage cycle; therefore, no newly synthesized methionine is needed for ethylene biosynthesis. Finally, ACC is oxidized by ACC oxidase (ACO) to form ethylene. Among three key enzymes (SAMS, ACS, and ACO), ACS is considered as the rate-limiting step in ethylene biosynthesis. Synthesis of ACC by ACS is controlled by numerous environmental conditions, such as biotic, osmotic, and water deficit stresses (Dubois et al., 2018). In soybean, ethylene biosynthesis genes ( $A C S$ and $A C O$ ) were upregulated under water deficit, increasing ethylene levels (Arraes et al., 2015). The stability of ACS activity is induced by stress-related kinases, MPK3/6 (Dubois et al., 2018).

Ethylene signaling is perceived by the receptors localized on the membrane endoplasmic reticulum (Zemlyanskaya et al., 2017). In contrast to other hormone receptors on the plasma membrane, unusual localization of ethylene receptors does not affect the ethylene signaling since gaseous ethylene can freely diffuses into the cell across the cell wall. In Arabidopsis, five ethylene receptors were identified: ethylene response1 (ETR1), ETR2, ethylene response sensor1 (ERS1), ERS2, and ethylene insensitive4 (EIN4). It has been known that the ethylene receptors are negative regulators in signal transduction. In absence of ethylene, receptors are activated, and they suppress the ethylene signaling. Receptors directly interact with constitutive triple response1 (CTR1), which inactivates a membrane protein EIN2 by phosphorylating the Cterminal of EIN2. Also, EIN2 is negatively regulated by the F-box protein ETP1/2 (EIN2 targeting protein1/2). In nucleus, there are key TFs, EIN3/EIL1, which are degraded by the Fbox proteins EBF1/2 (EIN3-binding F-box). In presence of ethylene, the receptors are inactivated, subsequently CTR1 is inactivated, preventing phosphorylation of EIN2. C-terminal end of EIN2 (EIN2C) is cleaved off by unknown mechanisms, and it moves into nucleus where EIN2C activates EIN3/EIL1 by degrading EBF1/2. The activated TFs, EIN3/EIL1, initiate ethylene-mediated transcriptional cascade. They activate transcription of ethylene response 
genes, including functional genes which cause the corresponding response to ethylene. EIN3/EIL1 also upregulates the expression of genes belong to AP2/ERF family. Members in AP2/ERF have been discovered in only plants, and they have been reported to be involved in response to abiotic and biotic stresses (Gutterson and Reuber, 2004). AP2/ERF TFs has a specific binding AP2 domain, which bind the promotor of ethylene response genes to activate or suppress their expression.

\subsection{Practical and genetic approaches to enhance water deficit tolerance}

Practical applications developed based on the knowledge of physiological and metabolic responses in plants have been utilized to minimize damage from water stress. When plants open stomata to take up carbon dioxide, water escapes simultaneously through the same stomata. The amount of transpirational water loss is estimated about $97 \%$ of total water absorbed by the root system (Taiz and Zeiger, 2010). To withstand water stress, plants induce stomatal closure to limit transpirational water loss by ABA-signaling pathways. Plants synthesize ABA under water stress, which triggers expressions of water stress-related genes and causes stomatal closure (Taiz and Zeiger, 2010). Preventing water loss from leaves could be an efficient method to help plants maintain water balance under water stress. Stomata can be physically blocked or physiologically closed by chemical treatments. Chemicals, known as antitranspirants, can be applied to plants to enhance tolerance to water stress (Odlum and Colombo, 1987). These compounds could contain waxes, resins, or polymers to physically block stomata or ABA to physiologically induce stomatal closure (Goreta et al., 2007). Such antitranspirants have shown positive effects on the enhancement of water stress tolerance by reducing transpirational water loss in vegetables, turf, and ornamental crops (Anderson and Kreith, 1978; Shinohara and Leskovar, 2014; Waterland et al., 2010a).

In addition to practical strategies, bioengineering has emerged as a permanent approach for developing crops with desired traits (Fang and Xiong, 2015). The outcome of physiological and molecular investigation of plants' response to water stress has allowed us to develop useful strategies for improving water stress tolerance in a limited number of crops. Studies have shown that manipulating regulatory genes resulted in increased tolerance to various abiotic stresses including water deficit stress (Golldack et al., 2011; Joshi et al., 2016). Especially, regulatory genes mediating water stress responses have been identified and characterized. Manipulating 
regulatory genes, protein kinases (e.g., CPKs and MPKs), phosphatases, and TFs (e.g., AREBs/ABFs, and ERFs), appeared to be effective in enhancing water stress tolerance (Fang and Xiong, 2015). Overexpression of protein kinases and TFs has led to an improvement of abiotic tolerance in several crops (Bhargava and Sawant, 2013). For example, rice transgenic

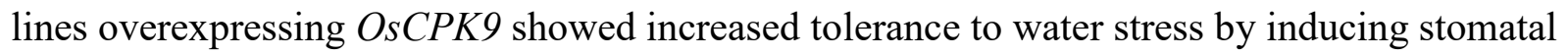
closure and by increasing osmolytes (proline and soluble sugar) contents (Wei et al., 2014). Transgenic rice overexpressing $A B F 3$ or $D R E B 2$ showed enhanced survivability and higher number of panicles compared to wild type plants under water stress (Bihani et al., 2011; Oh et al., 2005). However, most research was performed on Arabidopsis and a few of food crops (rice and tomato)(David et al., 2016). In my dissertation, petunia was used for transcriptome and functional analyses. Petunia belongs to Solanaceae family that includes the second largest economically important group of crops such as tomato, pepper, eggplant, tobacco, and potato. Petunia genome has been sequenced, and various tools for genetic and molecular biology research are readily available. Investigation of petunia as a model system will expand our knowledge on molecular mechanisms of water deficit stress responses in floriculture and vegetable crops. 


\section{Chapter 2 Evaluation of antitranspirants for enhancing temporary water stress tolerance in bedding ${ }^{1}$}

\subsection{Abstract}

Water stress during shipping and retailing reduces the postproduction quality and marketability of bedding plants. Antitranspirants can temporarily prevent plants from wilting by either physically blocking stomata or physiologically inducing stomatal closure, limiting transpirational water loss from leaves. The goal of this research was to evaluate the efficacy of commercially available antitranspirants on enhancing temporary water stress tolerance in bedding plants. Two physical antitranspirants, $\beta$-pinene polymer $(\beta \mathrm{P})$ and vinyl-acrylic polymer (VP), and three physiological antitranspirants, two sugar alcohol-based compounds (SACs) and a biologically active form of abscisic acid (s-ABA) were applied to begonia (Begonia semperflorens-cultorum Hort.), new guinea impatiens (Impatiens hawkeri Bull.), impatiens (Impatiens walleriana Hook.), petunia (Petunia $\times$ hybrida Hort.), african marigold (Tagetes erecta L.), and french marigold (Tagetes patula L.). Physical antitranspirants were sprayed on foliage and physiological antitranspirants were drenched to the media. All antitranspirants were applied at half $(0.5 \mathrm{X})$, equal to $(1 \mathrm{X})$, or twice $(2 \mathrm{X})$ the manufacturer's recommended rate. Extended shelf life was observed when $\beta$ P or s-ABA was applied. Treatment with $\beta$ P increased the shelf life of impatiens and african marigold by 1 and 1.3 days compared to control plants, respectively. The application of $\beta \mathrm{P}$ at $2 \mathrm{X}$ was more effective at delaying visual wilting than at lower rates $(0.5 \mathrm{X}$ and $1 \mathrm{X})$ in african marigold. Applications of s-ABA delayed wilting by 1.3 to 3.7 days in all tested cultivars. The shelf lives of impatiens and petunia treated with s-ABA at $2 \mathrm{X}$ were extended the most by 3.7 and 3.0 days compared to control plants, respectively. A rapid reduction of stomatal conductance was observed within 4 hours of $\beta \mathrm{P}$ or s-ABA application in plants showing delayed wilting symptoms. s-ABA treatment appeared to cause marginal leaf chlorosis in impatiens, while application of $\beta$ P damaged the opened flowers in all tested cultivars. The application of VP or SACs did not extend shelf life in any treated plants. These results suggest that foliage application of $\beta \mathrm{P}$ on selected species and treatment with s-ABA on most of species would allow bedding plants to withstand water deficit during shipping and/or retailing.

${ }^{1}$ This study is published in HortTechnology. Park, S., S.A. Mills, Y. Moon, and N.L. Waterland. 2016. Evaluation of antitranspirants for enhancing temporary water stress tolerance in bedding plants. HortTechnology 26:444-452. 


\subsection{Introduction}

Ornamental bedding plants represent the largest sector of the floriculture industry in the Unites States and have a wholesale value of $\$ 1.96$ billion accounting for $45 \%$ of all floriculture crops (USDA, 2014). In the last decade, there has been a shift in the retailing of ornamental crops. Customers tend to purchase bedding plants more in mass market retailers or superstores and general retail outlets (such as supermarkets) than in traditional garden centers and florists because of convenience and lower prices (Yue and Behe, 2008). In addition, the major growers have moved their production into areas characterized by lower labor cost and more favorable climatic conditions in order to reduce the cultivation cost (Ferrante et al., 2015). As a result, the location of crop production could be further away from markets, forcing plants to spend an extended period of time without proper irrigation during shipping and/or retailing (Waterland et al., 2010a; Weaver and van Iersel, 2014). Additionally, during postproduction periods, plants are often exposed to adverse environmental conditions, including high temperatures and inadequate irrigation, which accelerate substrate drying and plant wilting. Crop losses caused by these poor postproduction conditions are estimated to result in 5\% to $20 \%$ of unsalable crops (Healy, 2009), and water stress is one of the major causes of diminished aesthetic quality and salability of plants. Therefore, it is highly desired to minimize crop damages caused by water deficit to maintain high quality and prolong longevity of bedding plants during postproduction.

Water stress causes plants to synthesize a phytohormone called abscisic acid (ABA) in the root system, and it is translocated to leaves through the transpiration stream (Taiz and Zeiger, 2010). When ABA reaches guard cells, it binds to ABA receptors that activate an ion efflux, which reduces turgor pressure in the guard cells. Due to loss of turgidity, the guard cells become flaccid and stomata are closed. Closing of stomata inhibits transpiration and allows the plant to withstand water stress by decreasing water loss. Utilizing this principle, growers can use antitranspirants to reduce transpiration, thereby limiting water loss during shipping and retailing (Iriti et al., 2009; Odlum and Colombo, 1987; Waterland et al., 2010b).

Antitranspirants are chemical compounds that increase water stress tolerance by preventing transpirational water loss in plants. These compounds can be classified into two major groups based on their mode of action, physical and physiological antitranspirants (Anderson and Kreith, 1978; Shinohara and Leskovar, 2014; Waterland et al., 2010a). Physical antitranspirants contain waxes, resins, latexes, or polymers that coat the leaf surface and minimize water loss from the 
plant by blocking stomata (Goreta et al., 2007). Such physical antitranspirants have shown positive effects on water stress tolerance in pepper (Capsicum annuиm)(del Amor et al., 2010), peach tree (Prunus persica)(Steinberg et al., 1990), and herbaceous plants (Anderson and Kreith, 1978). Physiological antitranspirants minimize transpiration by inducing plants to close stomata. These compounds may contain ABA or other chemicals that increase the ABA concentration in plants (Waterland et al., 2010b). Exogenous application of ABA has enhanced water stress tolerance in various horticultural crops (Agehara and Leskovar, 2012; Astacio and van Iersel, 2011; Goreta et al., 2007; Shinohara and Leskovar, 2014). Goreta et al. (2007) found that foliar application of ABA enhanced water deficit tolerance of pepper, which was attributed to decreased stomatal conductance $\left(g_{\mathrm{s}}\right)$ and increased leaf water potential. Overall, antitranspirants have been shown to reduce wilting caused by water stress. However, some studies have demonstrated that plant responses to antitranspirants vary depending on species, concentrations of antitranspirants applied, developmental stages, and growing environmental conditions (Blanchard et al., 2007; Dunn et al., 2012; Shinohara and Leskovar, 2014; Waterland et al., 2010a).

Antitranspirants have been used to help plants withstand stress caused by water deficit, and many studies have focused on fruits, vegetables, turf, field crops, and woody plants. Little research has been conducted on the effect of antitranspirants on the postproduction quality of bedding plants. Furthermore, most research has evaluated individual antitranspirant and an efficacy comparison study among different products of physical and physiological antitranspirants is lacking. The physical antitranspirants in this study contained either $\beta$-pinene polymer $(\beta \mathrm{P})$ or vinyl-acrylic polymer (VP) as a coating agent and the physiological antitranspirants were two sugar alcohol-based compounds (SAC1 and SAC2), which are supposed to increase the concentration of $\mathrm{ABA}$ in plants, and a biologically active form of $\mathrm{ABA}$ (s-ABA). SAC1 contains xylitol, and SAC2 contains polyhydric alcohol and extracts from seaweed [e.g., red algae (Gracilaria sp.)], corn (Zea mays), and berries [e.g., brambles (Rubus sp.), blueberry (Vaccinium sp.), strawberry (Fragaria $\times$ ananassa)]. The goal of this research was to evaluate the efficacy of these commercially available antitranspirants on enhancing water stress tolerance in bedding plants. 


\subsection{Materials and methods}

Expt. 1: Shelf life of antitranspirant-treated bedding plants.

Eight cultivars of six popular bedding plant species were selected in this experiment. Four week old seedlings of 'Bada Bing Rose' begonia, 'Harmony Spicy Peach' new guinea impatiens, 'Double Fiesta Ole Purple Stripe' impatiens, 'Madness Double Red' and 'Wave Pink' petunia, 'Antigua Yellow' and 'Taishan Orange' african marigold, and 'Bonanza Yellow' french marigold were obtained in 288-plug trays except for impatiens and new guinea impatiens (72plug tray) from Green Circle Growers Inc. (Oberlin, OH). All plants were transplanted into 4inch pots with soilless greenhouse media (Sunshine ${ }^{\circledR}$ Mix \#1; Sun Gro Horticulture, Agawam, MA) in April 2013. They were grown in the greenhouse (Morgantown, WV) under natural irradiance with supplemental lighting. High-pressure sodium lamps (600W HS200 deep reflector; Hortilux, Pijnacker, The Netherlands) were used for supplemental lighting when natural radiance fell below $50 \mathrm{~W} \cdot \mathrm{m}^{-2}$. The average photosynthetic photon flux density (PPFD) was $412 \mu \mathrm{mol} \cdot \mathrm{m}^{-2} \cdot \mathrm{s}^{-1}$ from 0630 to $2030 \mathrm{HR}$ daily [mean daily light integral (DLI) $=20.8$ $\left.\mathrm{mol} \cdot \mathrm{m}^{-2} \cdot \mathrm{d}^{-1}\right]$. Mean greenhouse temperatures were $23.1 / 19.6 \pm 2.9 / 2.3{ }^{\circ} \mathrm{C}$ day/night (mean $\pm \mathrm{SD}$ ) with daytime relative humidity of $76.3 \% \pm 13.7 \%$. Plants were fertilized with $15 \mathrm{~N}-2.2 \mathrm{P}-12.5 \mathrm{~K}$ (Peter ${ }^{\circledR}$ Excel Cal-Mag; Everris, Marysville, $\mathrm{OH}$ ) at $200 \mathrm{mg} \cdot \mathrm{L}^{-1}$ nitrogen $(\mathrm{N})$. This was reduced to $100 \mathrm{mg} \cdot \mathrm{L}^{-1} \mathrm{~N} 1$ week before treatment.

All bedding plants were treated with antitranspirants when they reached a marketable stage of at least one open flower per plant. Begonia, new guinea impatiens, and impatiens were treated in June 2013, and marigold and petunia were treated in July 2013. Plants were irrigated with deionized (DI) water to container capacity $12 \mathrm{~h}$ before treatment. Physical antitranspirants were sprayed on the top and the underside of the plant canopy $(\approx 35 \mathrm{~mL}$ per plant $)$ with a pressurized sprayer (Regulator Bak-Pak; H.D. Hudson, Chicago, IL), and physiological antitranspirants were drenched in substrates $(60 \mathrm{~mL}$ per pot). Control treatments for physical and physiological antitranspirant applications were sprayed and drenched with DI water, respectively, and then they were irrigated daily with $100 \mathrm{mg} \cdot \mathrm{L}^{-1} \mathrm{~N}$ (irrigated control) or water was withheld (waterstressed control) during the period of the experiment. The physical antitranspirants used were $\beta \mathrm{P}$ (Wilt-Pruf ${ }^{\mathbb{B}}$; Wilt-Pruf Products, Inc., Essex, CT) and VP (Moisturin; WellPlant, Inc., Sparks, $\mathrm{NV}$ ). The physiological antitranspirants used were SAC1 (Stasis ${ }^{\mathrm{TM}}$; Natural Industries, Inc., Houston, TX), SAC2 (Root-Zone ${ }^{\mathrm{TM}}$; GSI Horticultural, Bend, OR), and s-ABA (ConTego ${ }^{\mathrm{TM}}$, 
VBC-30101, Valent BioSciences Corp., Libertyville, IL). All antitranspirants were applied at either half ( $0.5 \mathrm{X})$, equal to $(1 \mathrm{X})$, or twice $(2 \mathrm{X})$ the manufacturer's recommended application rate (Table 2.1). Plants were held in the greenhouse under the previously described environmental conditions for subsequent evaluations. Half of plants treated with each antitranspirant had water withheld (water-stressed) until all treated plants reached a visual wilt status rating of 3 or below (unmarketable) as described by Waterland et al. (2010c). Wilt status ratings were from 1 to 5 with 5 being completely turgid, 4 soft to the touch but still upright, 3 starting to wilt, 2 severely wilted, and 1 wilted to the point that leaves are dried and desiccated (Waterland et al., 2010a). The other half were irrigated daily with $100 \mathrm{mg} \cdot \mathrm{L}^{-1} \mathrm{~N}$ (irrigated daily) to determine whether antitranspirants caused any side effect on plants.

Visual observations of wilt status were taken daily. Evaluation of wilt status was started just before the application of antitranspirants. Evaluation was continued until all plants reached a visual wilt status rating of 3 or below. The shelf life of water-stressed plants was calculated as the number of days from the initiation of water being withheld until plants reached a wilt status rating of 3 (Waterland et al., 2010c).

Expt. 2: Wilt status and stomatal conductance of antitranspirant-treated bedding plants.

Four-week-old seedlings of 'Harmony Spicy Peach' new guinea impatiens were obtained in 72-plug trays from Green Circle Growers Inc. in April 2014. Seeds of 'Antigua Yellow' and 'Taishan Orange' african marigold, and 'Ultra Red' petunia were sowed in a 288-plug tray in March 2014. 'Ultra Red' petunias were used in Expt. 2 because 'Madness Double Red' and 'Wave Pink' petunias used in Expt. 1 were unavailable. All seedlings were transplanted into 4inch pots in April 2014 and grown in the greenhouse as described previously. Average PPFD was $304 \mu \mathrm{mol} \cdot \mathrm{m}^{-2} \cdot \mathrm{s}^{-1}$ from 0630 to $2030 \mathrm{HR}$ daily $\left(\mathrm{DLI}=15.3 \mathrm{~mol} \cdot \mathrm{m}^{-2} \cdot \mathrm{d}^{-1}\right.$ ). Average greenhouse temperatures were $23.7 / 18.1 \pm 3.0 / 2.5^{\circ} \mathrm{C}$ day/night (mean $\pm \mathrm{SD}$ ) with daytime relative humidity of $72.6 \% \pm 16.1 \%$. Based on the observations from Expt. 1, one physical ( $\beta \mathrm{P}$ ) and a physiological (s-ABA) antitranspirant were selected for the additional experiment at the recommended rate (1X). An additional treatment of 'Antigua Yellow' and 'Taishan Orange' african marigold with $\beta \mathrm{P}$ at $2 \mathrm{X}$ was performed. Antitranspirants were treated to new guinea impatiens and marigold in July 2014 and petunia in August 2014. $\beta$ P was sprayed $(\approx 35 \mathrm{~mL}$ per plant), and s-ABA was drenched (60 mL per pot). Plants were watered to container capacity $12 \mathrm{~h}$ before application. Control plants and antitranspirant-treated plants were either irrigated or 
water-stressed as described above. After all water-stressed plants reached a wilt status rating of 3 or below, they were rewatered daily to determine if there was any damage by antitranspirant. Visual wilt status of stressed plants was evaluated as described previously.

Stomatal conductance was measured with a portable photosynthesis system (LI-6400XT; LICOR Inc., Lincoln, NE). Three fully expanded leaves per plant were tagged for measurements. Stomatal conductance measurements were taken $1 \mathrm{~d}$ before treatment, $4 \mathrm{~h}$ after the treatment, daily until all plants showed visual wilting, and $3 \mathrm{~d}$ after plants were rewatered. A leaf was placed into a LED light source chamber (6400-02B; LI-COR Inc., Lincoln, NE). Environmental conditions in the chamber were set at $1,000 \mu \mathrm{mol} \cdot \mathrm{m}^{-2} \cdot \mathrm{s}^{-1} P P F D, 400 \mu \mathrm{mol} \cdot \mathrm{mol}^{-1} \mathrm{CO}_{2}$, and $25^{\circ} \mathrm{C}$ as the block temperature. Reading were conducted from 1000 to 1400 HR. Data are the means of measurements from three replications (or 3 plants), with three leaves measured per plant ( $n=3)$.

\section{Statistical analysis}

Experiments were conducted as a randomized complete block design with three replications $(\mathrm{n}=3)$. Analysis of variance (ANOVA) was performed by SAS version 9.3 (SAS Institute, Inc., Cary, NC). Bedding plants were blocked by replication based on plant position in the greenhouse and watering regimen (irrigated daily vs. water-stressed). Differences among the treatment means were assessed by Tukey's test at $P \leq 0.05$.

\subsection{Results}

\section{Expt. 1: Shelf life of antitranspirant-treated bedding plants.}

The application of $\beta \mathrm{P}$ at manufacturer's recommended rate (1X) delayed wilting symptoms in impatiens and two cultivars of african marigold increasing shelf life by 1 to $1.3 \mathrm{~d}$ as compared with stressed controls, while other species of plants did not show any extension of shelf life with $\beta$ P (Table 2.2). All tested bedding plants showed delayed visible wilting by s-ABA treatment and extended shelf life by 1.3 to $3.7 \mathrm{~d}$ depending on the cultivar (Table 2.2). In contrast to $\beta \mathrm{P}$ or sABA treatment, shelf life extension was not observed in any species treated with VP or SACs. Among the three rates of application (0.5X, $1 \mathrm{X}$, and $2 \mathrm{X})$, longer shelf life extension was observed when a higher rate $(2 \mathrm{X})$ of antitranspirant was applied compared to lower rates $(0.5 \mathrm{X}$ and $1 \mathrm{X}$ ) in 'Taishan Orange' african marigold treated with $\beta \mathrm{P}$ and 'Wave Pink' petunia treated with s-ABA (Table 2.3). Impatiens treated with s-ABA had longer shelf life extension at $1 \mathrm{X}$ and $2 \mathrm{X}$ than that at $0.5 \mathrm{X}$ (Table 2.3). All other plant species treated with $\beta \mathrm{P}$ or s-ABA and any of the 
plants treated with VP or either SACs had no difference in extension of shelf life regardless of application rates (data not shown). Overall, the longest shelf life extension was observed in 'Taishan Orange' african marigold treated with $\beta \mathrm{P}$ at $2 \mathrm{X}$ by $2 \mathrm{~d}$, and in impatiens and new guinea impatiens by almost $4 \mathrm{~d}$ when treated with s-ABA at $2 \mathrm{X}$ and $1 \mathrm{X}$, respectively (Tables 2.2 and 2.3).

Expt. 2: Wilt status and stomatal conductance of antitranspirant-treated bedding plants.

Applications of $\beta$ P and s-ABA, which showed the extended shelf life in Expt. 1, were performed to examine if there was a relationship between wilt status and $g_{s}$. Two cultivars of african marigold, new guinea impatiens, and 'Ultra Red' petunia were treated with $\beta$ P and s$\mathrm{ABA}$ at the recommended rate (1X). Additionally, the application of $\beta \mathrm{P}$ at $2 \mathrm{X}$ was included in both cultivars of african marigold since the longest shelf life was observed at that rate in Expt. 1 (Table 2.3).

As in Expt. 1, the application of $\beta \mathrm{P}$ delayed wilting symptoms in african marigold, but not in new guinea impatiens and petunia (Fig. 2.1). 'Antigua Yellow' african marigold treated with $\beta P$ at $1 \mathrm{X}$ and $2 \mathrm{X}$ did not show any visible wilting symptom on $2 \mathrm{~d}$ after treatment, while stressed control plants had a lower wilt status rating on the same day (Fig. 2.1B). A different african marigold cultivar, Taishan Orange, treated with the higher rate of $\beta \mathrm{P}(2 \mathrm{X})$ showed less visible wilting than stressed control or plants treated with $1 \mathrm{X}$ of $\beta \mathrm{P}$ on 2 and $3 \mathrm{~d}$ after treatment (Fig. 2.1C). Petunia did not show any difference in visual wilting symptoms between stressed control and $\beta$ P-treated plants during the period of water deficit stress (Fig. 2.1D).

Stomatal conductance decreased $45 \%$ to $73 \%$ compared to that of control plants within $4 \mathrm{~h}$ after treatment with $\beta \mathrm{P}$ in both irrigated daily and water-stressed new guinea impatiens and two cultivars of african marigold (Fig. 2.1A-C). As water stress progressed, $g_{\mathrm{s}}$ of stressed controls declined and their value became similar to that of $\beta$ P-treated plants 4,2 , and $2 \mathrm{~d}$ after treatment in new guinea impatiens, 'Antigua Yellow' african marigold, and 'Taishan Orange' african marigold, respectively (Fig. 2.1A-C). The application of $\beta$ P reduced $g_{s}$ in water-stressed petunia $4 \mathrm{~h}$ after treatment, but it became similar to control $1 \mathrm{~d}$ after treatment (Fig. 2.1D). Comparing the application rates of $\beta \mathrm{P}$ in two cultivars of african marigolds, there was no $g_{\mathrm{s}}$ difference between plants treated at $2 \mathrm{X}$ and $1 \mathrm{X} 4 \mathrm{~h}$ after treatment (Fig. 2.1B and C).

All plants treated with s-ABA had delayed visual wilting (Fig. 2.2) and exhibited a wilt status rating over 4 for $4 \mathrm{~d}$ (new guinea impatiens) and $3 \mathrm{~d}$ (african marigold and petunia) after the 
application (Fig. 2.2). Four hours after s-ABA application, reduced $g_{\mathrm{s}}$ was observed in all cultivars tested under irrigated daily and water-stressed conditions (Fig. 2.2). Stomatal conductance of water-stressed control plants reached nearly the same level as s-ABA-treated plants 2 to $4 \mathrm{~d}$ after treatment (Fig. 2.2).

\subsection{Discussion}

Two physical and three physiological antitranspirants were evaluated for enhancing temporary water stress tolerance in eight popular cultivars of bedding plants. Among the five antitranspirants examined, only $\beta \mathrm{P}$ in certain cultivars and s-ABA in all cultivars showed positive effects on enhancing water stress tolerance. Three other antitranspirants (VP and SACs) were not effective and consequently further evaluation of VP and SACs was not performed.

A sharp decline of $g_{\mathrm{s}} 4 \mathrm{~h}$ after application of $\beta \mathrm{P}$ and $\mathrm{s}-\mathrm{ABA}$ compared to controls indicated that application of $\beta \mathrm{P}$ effectively blocked stomata or s-ABA induced stomatal closure at the early stage of application in those plants (Figs. 2.1 and 2.2). The delay in wilting was likely due to stomatal closure, and the subsequent reduction in water loss, thus delaying the loss in leaf turgidity (Figs. 2.1 and 2.2). A rapid reduction of $g_{\mathrm{s}}$ resulting from application of antitranspirants helped to maintain a high water status under water stress and thus improved water stress tolerance (Astacio and van Iersel, 2011; Kim and van Iersel, 2011; Shinohara and Leskovar, 2014; Waterland et al., 2010b). Additionally, Anderson and Kreith (1978) reported that $\beta P$ treatment resulted in initial reduction of transpiration rate by reducing $g_{\mathrm{s}}$ of sweetclover (Melilotus officinalis (L.) Pall.) leaves. The application of $\beta$ P caused a reduction in water use of peach trees by $40 \%$ immediately after treatment, with a subsequent decrease in water use by $30 \%$

for the next $30 \mathrm{~d}$ (Steinberg et al., 1990). Although $\beta$ P treatment reduced $g_{\mathrm{s}}$ within $4 \mathrm{~h}$ in petunia, transpiration resumed $1 \mathrm{~d}$ after treatment, as indicated by similar $g_{\mathrm{s}}$ to control, and it did not delay wilting (Fig. 2.1D). Therefore, the rapid and sustained reduction in $g_{\mathrm{s}}$ by antitranspirants prior to water stress seemed to greatly reduce transpirational water loss during the beginning of water stress period, thus delaying wilting symptoms. As $\beta \mathrm{P}$ and s-ABA-treated plants were irrigated daily, $g_{s}$ gradually increased to the same level of irrigated controls (Figs. 2.1 and 2.2). Thus, the efficacy of antitranspirants was diminished as plants were irrigated or as time passed.

Our findings also support the idea that the effects of $\beta \mathrm{P}$ on enhancing water stress tolerance appeared to be species-dependent. Application of $\beta \mathrm{P}$ did not extend shelf life universally, but 
only in impatiens and african marigold, among the six species tested when they were treated at the manufacturer's recommend rate (Table 2.2). Studies have reported that physical antitranspirants resulted in different responses depending on species (Davies et al., 2005; Hummel, 1990). Species differences in response to physical antitranspirants might be associated with differences in shape, size, and density of trichomes (Goreta et al., 2007). Since physical antitranspirants are sprayed on the leaf surface to coat the stomata with a thin film of the chemicals, trichome patterns might affect the chemical's adhesion and retention on leaves differently (Palliotti et al., 2010; Pathan et al., 2009). In artichoke (Cynara cardunculus L.), physical antitranspirants were not effective to mitigate water stress, presumably due to the dense glandular trichomes (Shinohara and Leskovar, 2014). Indeed, the petunia cultivar evaluated in this research has hirsute leaves, while african marigold has rather glabrous leaves. New guinea impatiens tested also have fewer trichomes than petunia and reduced $g_{\mathrm{s}}$ was observed when $\beta \mathrm{P}$ was applied (Fig. 2.1A). The denser trichomes might have prevented $\beta \mathrm{P}$ from forming a thin film layer on the stomata. Species-dependent response to $\beta \mathrm{P}$ in this experiment may have been due to the different characteristics of surface trichome patterns.

Application of s-ABA enhanced water stress tolerance in all plants tested, and the range of extended shelf life varied from 1.3 to $3.7 \mathrm{~d}$ depending on species and cultivars. Responses to ABA treatments have been shown to vary according to species (Blanchard et al., 2007; Waterland et al., 2010a). Blanchard et al. (2007) reported that sprench application of s-ABA at 125 or $250 \mathrm{mg} \cdot \mathrm{L}^{-1}$ delayed wilting by 1.1 to $5.8 \mathrm{~d}$ in 'Harmony Grape' new guinea impatiens, but no significant effect of s-ABA on shelf life was observed in 'Tempo Lavender' impatiens or 'Vabana' bacopa (Sutera cordata Roth.). In 'Double Fiesta Ole Purple Stripe' impatiens, application of s-ABA at 250,500 , and $1000 \mathrm{mg} \cdot \mathrm{L}^{-1}$ extended the shelf life by 1.7 to $3.7 \mathrm{~d}$ (Table 2.3). Longer shelf life of 'Xtreme Lavender' impatiens either drenched or sprayed with s-ABA was also observed by Waterland et al. (2010a). The differences between previously published reports and the result from this research may be due to different cultivar selection, s-ABA concentration, application method, and environmental conditions.

In contrast to $\beta \mathrm{P}$ and $\mathrm{s}-\mathrm{ABA}, \mathrm{VP}$ and $\mathrm{SACs}$ did not exhibit any positive or negative effect on shelf life extension in all eight cultivars at manufacturers' recommended rates (Table 2.2). VP had shown increased survival rate of green ash (Fraxinus pennsylvanica Marsh.)(Harris and Bassuk, 1995) and reduced water use in nursery trees (Englert et al., 1993) after transplanting. 
Those plants, however, were subjected to water stress by transplanting and might not have experienced similar levels of water stress as in the present study, which withheld water from container grown plants. Dunn et al. (2012) found that SACs delayed the visual wilting rating of herbaceous and woody ornamentals compared to non-treated controls, but in a species-dependent manner. The authors mentioned that the species-dependent responses might be due to the different application rates, retention, and accumulation of chemicals in soilless media. SACs are expected to lower water potential in growing media to induce water stress. In this research, all plants treated with either SAC showed no delay in wilting symptoms even at twice the recommended rate, indicating that $\mathrm{SACs}$ failed to trigger water stress response.

Although application of $\beta \mathrm{P}$ was effective in certain plant species, $\beta \mathrm{P}$ caused floral damage in all plants tested regardless of application rate (Fig. 2.3). Floral damage was first observed within a few hours after $\beta$ P treatment, and the floral damage seemed to accelerate flower senescence, resulting in poor quality of bedding plants. However, no damage was observed in shoots, flower buds, or leaves. Steinberg et al. (1990) reported that $\beta$ P did not significantly reduce growth or bud and fruit initiation in peach tree. Therefore, application of $\beta \mathrm{P}$ should be recommended prior to flower opening. On the other hand, s-ABA caused chlorosis on the margin of leaves in impatiens. Foliar chlorosis and leaf abscission have been frequently mentioned as side effects of ABA application (Agehara and Leskovar, 2012; Astacio and van Iersel, 2011; Kim and van Iersel, 2011; Waterland et al., 2010a; Waterland et al., 2010c; Weaver and van Iersel, 2014). Chlorosis has been known to accelerate with increasing ABA concentration (Agehara and Leskovar, 2012; Astacio and van Iersel, 2011; Weaver and van Iersel, 2014).

The efficacy of five antitranspirants to increase temporary tolerance to water stress was evaluated in six species of bedding plants. Among five antitranspirants, $\beta \mathrm{P}$ and s-ABA treatment enhanced temporary water stress tolerance in severely water-stressed plants by blocking and closing stomata, respectively. This explanation was supported by the observation that $g_{\mathrm{s}}$ was quickly decreased upon application of either antitranspirant. Consequently, shelf life was increased by 1 to $3.7 \mathrm{~d}$ depending on species and application rates. The application of $\beta \mathrm{P}$ or s$\mathrm{ABA}$ as an antitranspirant would allow some bedding plants to withstand temporary water stress during postproduction such as the shipping and retailing environments. However, the efficacy of $\beta \mathrm{P}$ appears to be species dependent in this research, possibly due to the difference in trichome patterns of leaf surface. Caution should also be given that the application of $\beta \mathrm{P}$ and s-ABA could 
cause floral damage or leaf chlorosis. Floriculture growers should evaluate the effects of antitranspirants on their crops to maximize aesthetic quality and longevity of their products without any side effects. 
Table 2.1. Manufacturer's recommended application rate of antitranspirants.

\begin{tabular}{|c|c|}
\hline Antitranspirant $^{\mathrm{Z}}$ & Rate $\left(\mathrm{mL} \cdot \mathrm{L}^{-1}\right)^{\mathrm{y}}$ \\
\hline$\beta \mathrm{P}$ & 100.0 \\
\hline VP & 100.0 \\
\hline $\mathrm{SAC} 1$ & 50.0 \\
\hline $\mathrm{SAC} 2$ & 7.8 \\
\hline s-ABA & 0.5 \\
\hline \multicolumn{2}{|c|}{${ }^{\mathrm{z}} \beta \mathrm{P}, \beta$-pinene polymer (Wilt-Pruf ${ }^{\circledR}$; Wilt-Pruf Products, Inc., } \\
\hline \multicolumn{2}{|c|}{ Essex, CT); VP, vinyl-acrylic polymer (Moisturin; WellPlant, } \\
\hline \multicolumn{2}{|c|}{ Inc., Sparks, NV); SAC1, sugar alcohol-based compound 1} \\
\hline \multicolumn{2}{|c|}{$\left(\right.$ Stasis $^{\mathrm{TM}} ;$ Natural Industries, Inc., Houston, TX); SAC2, } \\
\hline \multicolumn{2}{|c|}{ sugar alcohol-based compound 2 (Root-Zone ${ }^{\mathrm{TM}} ;$ GSI } \\
\hline \multicolumn{2}{|c|}{ Horticultural, Bend, OR); s-ABA, a biologically active form } \\
\hline \multicolumn{2}{|c|}{ of ABA (ConTego ${ }^{\mathrm{TM}}$, VBC-30101; Valent BioSciences Corp., } \\
\hline Libertyville, IL). & \\
\hline $\mathrm{mL} \cdot \mathrm{L}^{-1}=100$ & \\
\hline
\end{tabular}


Table 2.2. The number of days until the appearance of wilting symptom in eight cultivars of bedding plants treated with antitranspirants at manufacturer's recommended rate $(1 \mathrm{X})$ under water deficit stress ${ }^{\mathrm{z}}$.

\begin{tabular}{|c|c|c|c|c|c|c|c|}
\hline \multirow[b]{4}{*}{ Cultivar } & \multicolumn{7}{|c|}{ Type of antitranspirant } \\
\hline & \multicolumn{7}{|c|}{ Time before wilted $(\mathrm{d})$} \\
\hline & \multicolumn{3}{|c|}{ Physical $^{\mathrm{y}}$} & \multicolumn{4}{|c|}{ Physiological $^{\mathrm{x}}$} \\
\hline & Control & $\beta P$ & $\mathrm{VP}$ & Control & $\mathrm{SAC1}$ & $\mathrm{SAC} 2$ & S-ABA \\
\hline Bada Bing Rose begonia & $8.3 \mathrm{a}^{\mathrm{w}}$ & $8.3 \mathrm{a}$ & $9.0 \mathrm{a}$ & $8.7 \mathrm{~b}$ & $8.0 \mathrm{~b}$ & $8.7 \mathrm{~b}$ & $10.0 \mathrm{a}$ \\
\hline Harmony Spicy Peach new guinea impatiens & $2.0 \mathrm{a}$ & $2.0 \mathrm{a}$ & $2.3 \mathrm{a}$ & $2.0 \mathrm{~b}$ & $2.3 \mathrm{~b}$ & $2.0 \mathrm{~b}$ & $5.7 \mathrm{a}$ \\
\hline Double Fiesta Ole Purple Stripe impatiens & $2.0 \mathrm{~b}$ & $3.0 \mathrm{a}$ & $2.3 \mathrm{ab}$ & $2.3 \mathrm{~b}$ & $2.7 \mathrm{~b}$ & $2.0 \mathrm{~b}$ & $5.3 \mathrm{a}$ \\
\hline Madness Double Red petunia & $3.0 \mathrm{a}$ & $3.7 \mathrm{a}$ & $2.7 \mathrm{a}$ & $2.7 \mathrm{~b}$ & $3.3 \mathrm{~b}$ & $3.3 \mathrm{~b}$ & $6.0 \mathrm{a}$ \\
\hline Wave Pink petunia & $2.7 \mathrm{a}$ & $3.0 \mathrm{a}$ & $2.7 \mathrm{a}$ & $3.0 \mathrm{~b}$ & $3.0 \mathrm{~b}$ & $3.3 \mathrm{ab}$ & $4.7 \mathrm{a}$ \\
\hline Antigua Yellow african marigold & $2.0 \mathrm{~b}$ & $3.3 \mathrm{a}$ & $2.0 \mathrm{~b}$ & $2.3 \mathrm{~b}$ & $2.0 \mathrm{~b}$ & $2.3 \mathrm{~b}$ & $5.3 \mathrm{a}$ \\
\hline Taishan Orange african marigold & $2.0 \mathrm{~b}$ & $3.0 \mathrm{a}$ & $2.3 \mathrm{ab}$ & $2.0 \mathrm{~b}$ & $2.0 \mathrm{~b}$ & $2.0 \mathrm{~b}$ & $5.0 \mathrm{a}$ \\
\hline Bonanza Yellow french marigold & $2.0 \mathrm{a}$ & $2.0 \mathrm{a}$ & $2.0 \mathrm{a}$ & $2.0 \mathrm{~b}$ & $2.0 \mathrm{~b}$ & $2.0 \mathrm{~b}$ & $4.7 \mathrm{a}$ \\
\hline
\end{tabular}

${ }^{\mathrm{z}}$ Values are the number of days from the time antitranspirants were applied until plants started showing visible symptoms of wilting.

Values are means of three replications $(n=3)$.

${ }^{\mathrm{y}}$ Control, sprayed with deionized water once on Day 0 and then water was withheld; $\beta \mathrm{P}, \beta$-pinene polymer; VP, vinyl-acrylic polymer.

${ }^{\mathrm{x}}$ Control, drenched with deionized water once on Day 0 and then water was withheld; SAC1, sugar alcohol-based compound 1; SAC2, sugar alcohol-based compound 2; s-ABA, a biologically active form of abscisic acid.

${ }^{\text {w }}$ Mean separation among either physical or physiological antitranspirants within cultivars by Tukey's significance test at $P \leq 0.05$. 
Table 2.3. Shelf life extension of two cultivars of african marigold treated with $\beta$-pinene polymer ( $\beta \mathrm{P})$, and impatiens and petunia treated with a biologically active form of abscisic acid (s-ABA) at three rates under water deficit stress ${ }^{\mathrm{z}}$.

\begin{tabular}{|c|c|c|c|}
\hline Antitranspirant $^{y}$ & Cultivar & Rate $^{\mathrm{x}}$ & Shelf life extension $(d)$ \\
\hline \multirow{8}{*}{$\beta \mathrm{P}$} & Antigua Yellow african marigold & $0.5 \mathrm{X}$ & $0.7 \mathrm{~b}^{\mathrm{w}}$ \\
\hline & & $1 \mathrm{X}$ & $1.3 \mathrm{ab}$ \\
\hline & & $2 \mathrm{X}$ & $1.7 \mathrm{a}$ \\
\hline & Significance & & $*$ \\
\hline & Taishan Orange african marigold & $0.5 \mathrm{X}$ & $0.7 \mathrm{~b}$ \\
\hline & & $1 \mathrm{X}$ & $1.0 \mathrm{~b}$ \\
\hline & & $2 \mathrm{X}$ & $2.0 \mathrm{a}$ \\
\hline & Significance & & $*$ \\
\hline \multirow[t]{8}{*}{ s-ABA } & Double Fiesta Ole Purple Stripe impatiens & $0.5 \mathrm{X}$ & $1.7 \mathrm{~b}$ \\
\hline & & $1 \mathrm{X}$ & $3.0 \mathrm{a}$ \\
\hline & & $2 \mathrm{X}$ & $3.7 \mathrm{a}$ \\
\hline & Significance & & $* *$ \\
\hline & Wave Pink petunia & $0.5 \mathrm{X}$ & $2.0 \mathrm{~b}$ \\
\hline & & $1 \mathrm{X}$ & $1.7 \mathrm{~b}$ \\
\hline & & $2 \mathrm{X}$ & $3.0 \mathrm{a}$ \\
\hline & Significance & & $*$ \\
\hline
\end{tabular}

${ }^{\mathrm{z}}$ Values are the number of additional days from the time when the control plants began to wilt until the time antitranspirant-treated plants started to show visible symptoms of wilting. Values are means of three replications $(\mathrm{n}=3)$.

${ }^{y} \beta P, \beta$-pinene polymer; s-ABA, a biologically active form of abscisic acid.

${ }^{x}$ Rates were half $(0.5 \mathrm{X})$, equal to $(1 \mathrm{X})$, or twice $(2 \mathrm{X})$ the manufacturer's recommended application rate.

${ }^{\text {w }}$ Mean separation among three rates within cultivars by Tukey's significance test at $P \leq 0.05$.

*,** Significant at $P \leq 0.05$ or 0.01 , respectively. 


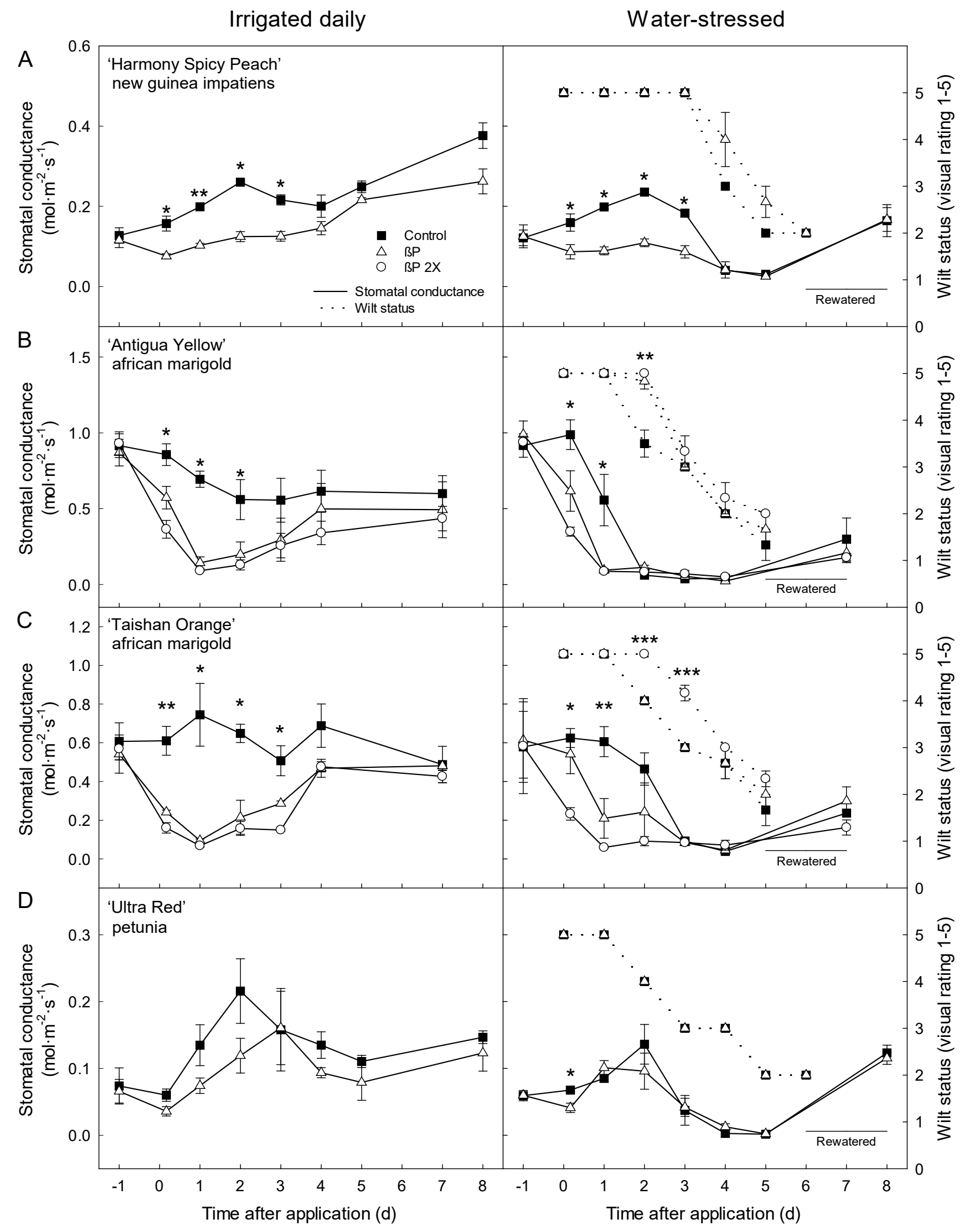


Fig. 2.1. Stomatal conductance and wilt status rating of $\beta$-pinene polymer $(\beta P)$-treated 'Harmony Spicy Peach’ new guinea impatiens (A), ‘Antigua Yellow' african marigold (B), 'Taishan Orange' african marigold (C), and 'Ultra Red' petunia (D). $\beta$ P was applied at the manufacturer's recommended rate $(1 \mathrm{X})$ in all tested cultivars. African marigolds were treated with $\beta \mathrm{P}$ at $1 \mathrm{X}$ and 2X. Half of plants were irrigated daily (left), and the other half had water withheld until plants showed wilt status rating of 3 and irrigation was resumed for $3 \mathrm{~d}$ (right). Stomatal conductance was measured $1 \mathrm{~d}$ before the application, $4 \mathrm{~h}$ after the application, daily until all plants showed wilt symptom, and $3 \mathrm{~d}$ after plants were rewatered. Irrigated controls had wilt status of 5 for the duration of the experiment, and water-stressed plants had a rating of 5 after $3 \mathrm{~d}$ rewatering period. Wilt status ratings were from 5 to 1 , where $5=$ completely turgid, $4=$ soft to touch but still upright, $3=$ starting to wilt, $2=$ severely wilted, and $1=$ wilted to the point that leaves are desiccated. Vertical bars are standard errors of the means with three replications $(\mathrm{n}=3) .{ }^{*}, * *$,

${ }^{* * *}$ Significant at $P \leq 0.05,0.01$, or 0.001 , respectively. 


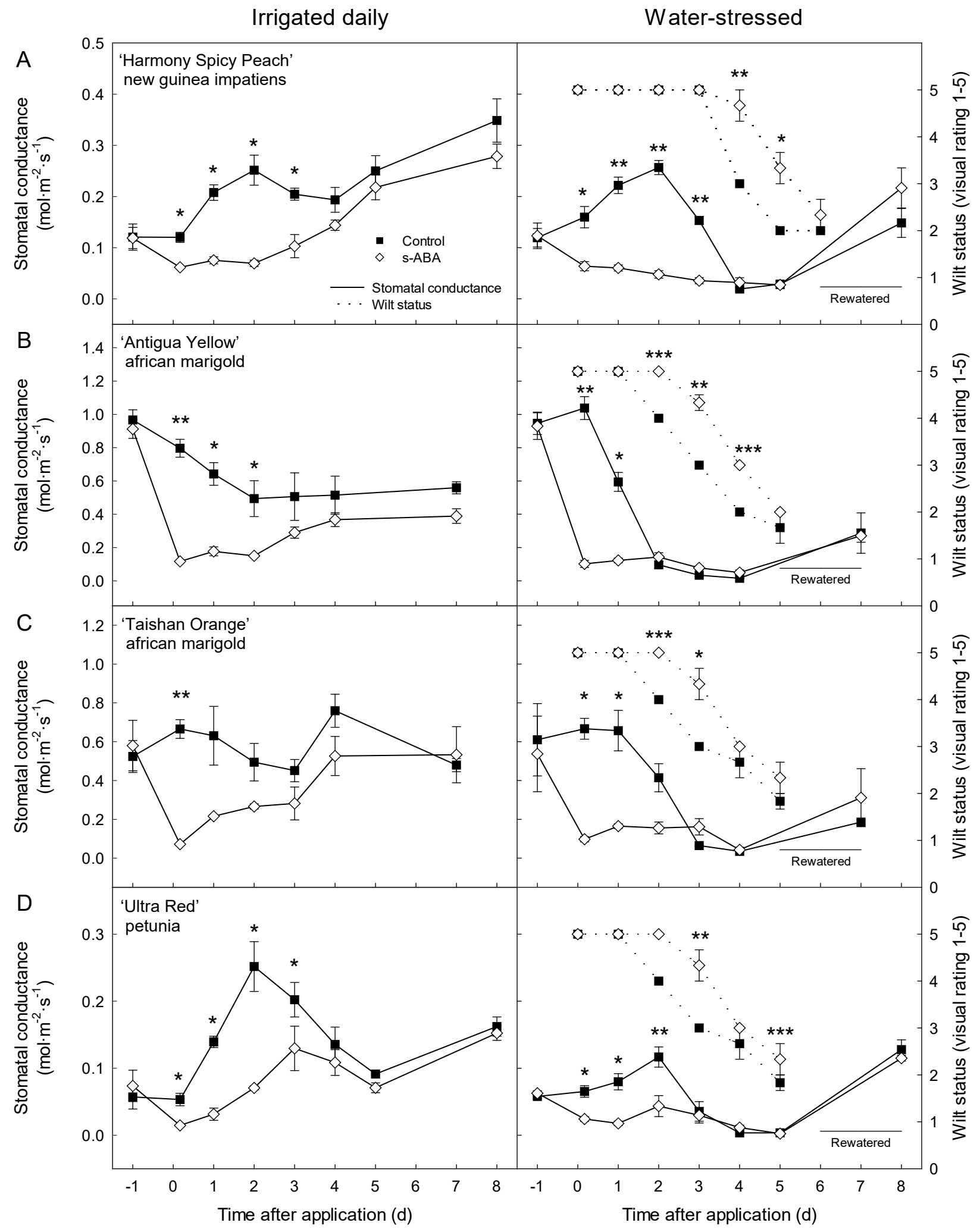


Fig. 2.2. Stomatal conductance and wilt status rating of a biologically active form of abscisic acid (s-ABA)-treated 'Harmony Spicy Peach' new guinea impatiens (A), 'Antigua Yellow' african marigold (B), 'Taishan Orange' african marigold (C), and 'Ultra Red' petunia (D). sABA was applied at the manufacturer's recommended rate (1X). Half of plants were irrigated daily (left), and the other half had water withheld until plants showed wilt status rating of 3 and irrigation was resumed for $3 \mathrm{~d}$ (right). Stomatal conductance was measured $1 \mathrm{~d}$ before the application, $4 \mathrm{~h}$ after the application, daily until all plants showed wilt symptom, and $3 \mathrm{~d}$ after plants were rewatered. Irrigated controls had wilt status of 5 for the duration of the experiment, and water-stressed plants had a rating of 5 after $3 \mathrm{~d}$ rewatering period. Wilt status ratings were from 5 to 1 , where $5=$ completely turgid, $4=$ soft to touch but still upright, $3=$ starting to wilt, 2 $=$ severely wilted, and $1=$ wilted to the point that leaves are desiccated. Vertical bars are standard errors of the means with three replications $(\mathrm{n}=3) .{ }^{*},{ }^{* *},{ }^{* * *}$ Significant at $P \leq 0.05,0.01$, or 0.001 , respectively. 


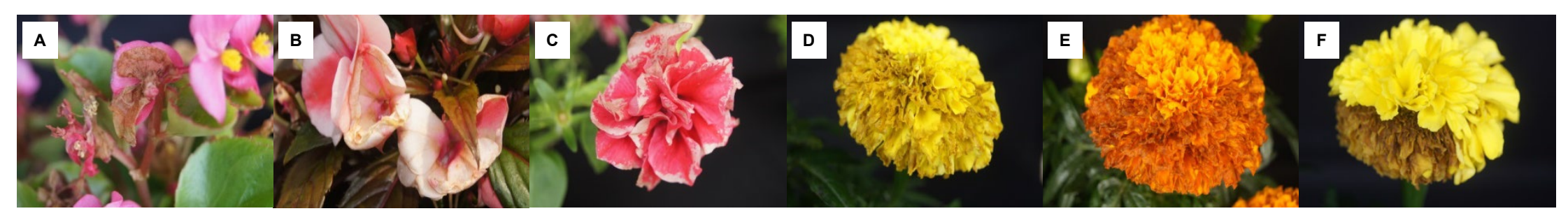

Fig. 2.3. Floral damage of bedding plants treated with $\beta$-pinene polymer $(\beta \mathrm{P})$ at $1 \mathrm{X}$. 'Bada Bing Rose' begonia (A), 'Harmony Spice Peach' new guinea impatiens (B), 'Madness Double Red' petunia (C), 'Antigua Yellow' african marigold (D), 'Taishan Orange' african marigold (E), and 'Bonanza Yellow' french marigold (F). 


\section{Chapter 3 Treatment of calcium chloride enhances water deficit tolerance in viola (Viola cornuta)}

\subsection{Abstract}

Water deficit stress during shipping and retailing can reduce postproduction shelf life and marketability of floriculture crops. To alleviate damage by water deficiency, plants need to limit transpirational water loss by inducing stomatal closure. Osmotic stress induces stomatal closure similar to the response to water deficit stress and could be used as a convenient tool to enhance water deficit stress tolerance. The objective of this research was to investigate whether osmotic treatment with a high concentration of chemical solutions could trigger a response to osmotic stress so that stomatal closure can be induced, resulting in enhanced water deficit stress tolerance in viola (Viola cornuta 'Sorbet XP Yellow'). Osmotic treatments with $\mathrm{CaCl}_{2}, \mathrm{Ca}\left(\mathrm{NO}_{3}\right)_{2}, \mathrm{NaCl}$, $\mathrm{NaNO}_{3}, \mathrm{BaCl}_{2}, \mathrm{Ba}\left(\mathrm{NO}_{3}\right)_{2}$, and mannitol were applied. Chemical treatments except $\mathrm{Ca}\left(\mathrm{NO}_{3}\right)_{2}$, $\mathrm{NaCl}$, and mannitol helped to delay wilting and extended shelf life up to 2.6 days compared to control. However, leaf necrosis was observed on the plants treated with $\mathrm{NaCl}, \mathrm{NaNO}_{3}, \mathrm{BaCl}_{2}$, $\mathrm{Ba}\left(\mathrm{NO}_{3}\right)_{2}$, and mannitol. $\mathrm{CaCl}_{2}$ was the most effective agent in delaying wilting under water deficit stress in viola without leaf necrosis. Compared to control, viola treated with $\mathrm{CaCl}_{2}$ at 200 and $300 \mathrm{mM}$ showed an increase in shelf life by 2.6 and 1.2 days, respectively. Additionally, stomatal conductance and leaf relative water content (RWC) were measured daily after $\mathrm{CaCl}_{2}$ application. Stomatal conductance was reduced by within $4 \mathrm{~h}$ after treatment with $\mathrm{CaCl}_{2}$ compared to that of control plants. Leaf RWC of control plants was dramatically reduced 3 days after treatment and fell below $50 \%$ on day 4 , while $\mathrm{CaCl}_{2}$-treated plants maintained higher leaf RWC (70-81\%) during water deficit period. These results indicated that osmotic treatment with the high concentration of $\mathrm{CaCl}_{2}$ caused stomatal closure, resulting in reduction of water loss and extension of shelf life under water deficit stress in viola. It will be interesting to see if pretreatment of high concentration of chemicals such as fertilizers prior to shipping could enhance tolerance to water deficit stress in bedding plants.

\subsection{Introduction}

Floriculture crops are often exposed to harsh environmental conditions due to high temperatures and irregular irrigation during shipping and retailing. Crop loss during postproduction period is estimated up to $20 \%$ of unsalable crops (Healy, 2009). These poor 
postproduction conditions often cause water deficit stress, leading to decreased aesthetic values and marketability (Armitage, 1993; Sharma et al., 2006).

When encountering water deficit stress, plants need to reduce transpiration to minimize water loss during water deficit period. Plants can regulate transpirational rate by increasing leaf stomatal resistance. Under the water deficit environment, plants synthesize a phytohormone, abscisic acid, and it triggers stomatal closure to prevent transpirational water loss (Taiz and Zeiger, 2010). Using this principle, artificially blocking stomatal or inducing stomatal closure using antitranspirants could be an efficient method to temporarily reduce water loss and maintain water balance. Applications of antitranspirants increased water deficit stress tolerance by reducing stomatal conductance (gs) in bedding plants (Park et al., 2016; Waterland et al., 2010a; Waterland et al., 2010b).

Stress tolerance can be also enhanced by imposing a single stressor in advance of later stresses. An earlier stress can result in an enhanced tolerance to future stresses by helping plants to respond more quickly and actively (Hossain et al., 2017; Li and Liu, 2016; Savvides et al., 2016). Among various abiotic stresses, water deficit and salt stresses are manifested primarily as osmotic stress to plants (Bartels and Sunkar, 2005; Wang et al., 2003). Both stresses reduce the soil water potential and lead to cellular dehydration, resulting in the disruption of homeostasis in the cell (Bartels and Sunkar, 2005). As a result, water deficit and salt stress activate common mechanisms of cellular response, such as osmotic adjustment and antioxidant activity, and trigger similar physiological changes to withstand stresses, such as stomatal closure (Bartels and Sunkar, 2005; Wang et al., 2003; Zhang et al., 2006). However, a pre-exposure to osmotic stress has not been studied for enhancing water deficit stress, especially focusing on stomatal closure and water loss.

Calcium is well-known as a secondary messenger involved in various physiological and biochemical processes in osmotic stress in plants (Taiz and Zeiger, 2010). Abiotic stresses elevate cytosolic calcium level in plant cells, and calcium mediates stress signal transduction and activates defense mechanisms (Tuteja and Mahajan, 2007). Exogenous application of calcium has shown to ameliorate the adverse effects of salt, cold, heat, and water deficit by modulating antioxidant, growth performance, photosynthetic efficiency, and osmolytes production (Shoresh et al., 2011; Tan et al., 2011; Upadhyaya et al., 2011; Xu et al., 2013a). However, no attempt has 
been made to determine whether application of calcium improves water deficit tolerance by promoting stomatal closure.

The hypothesis of this research was osmotic treatment with calcium would enhance water deficit tolerance in plants by inducing stomatal closure. The objectives of this research were to 1) determine the effects of osmotic treatment for enhancing water deficit tolerance in viola, and 2) identify the relationship between stomatal closure and water loss under water deficit in $\mathrm{CaCl}_{2}$ treated viola. Viola was selected in this experiment because it is one of the top popular bedding plants in the United States and it is very sensitive to water deficit stress.

\subsection{Materials and methods}

\section{Expt. 1: Effects of osmotic treatment on shelf life in Viola}

Seeds of Viola cornuta 'Sorbet XP Yellow' were sown in 288-plug tray on Oct. 13, 2017, and 3-week-old seedlings were transplanted into 11-cm pots with soilless media (Sunshine ${ }^{\circledR}$ Mix \#1; Sun Gro Horticulture, Agawam, MA). Plants were grown in the greenhouse (Morgantown, WV) under natural irradiance with supplemental high-pressure sodium lamps (600W HS200 deep reflector; Hortilux, Pijnacker, The Netherlands). The average photosynthetic photon flux density $(P P F D)$ was $197 \mu \mathrm{mol} \cdot \mathrm{m}^{-2} \cdot \mathrm{s}^{-1}$ from 0600 to $2000 \mathrm{HR}$ daily [mean daily light integral $\left.(D L I)=8.7 \mathrm{~mol} \cdot \mathrm{m}^{-2} \cdot \mathrm{d}^{-1}\right]$. Mean greenhouse temperatures were $21.7 / 17.3 \pm 3.6 / 2.3{ }^{\circ} \mathrm{C}$ day $/$ night (mean \pm SD) with mean relative humidity of $44.3 \%$. Plants were fertilized with $20 \mathrm{~N}-3 \mathrm{P}-19 \mathrm{~K}$ $\left(\right.$ Peter $^{\circledR}$ Professional Petunia Special; Everris NA, Marysville, OH) at $200 \mathrm{mg} \cdot \mathrm{L}^{-1}$ nitrogen (N). This was reduced to $100 \mathrm{mg} \cdot \mathrm{L}^{-1} \mathrm{~N}$ one week before treatment.

Thirteen-week-old violas were irrigated with deionized (DI) water to container capacity 12 hours before treatment. Control treatment was drenched with deionized water. Calcium treatments included $\mathrm{CaCl}_{2}$ and $\mathrm{Ca}\left(\mathrm{NO}_{3}\right)_{2}$. To distinguish whether physiological changes resulted from calcium treatment, additional sodium containing chemicals $\left(\mathrm{NaCl}, \mathrm{NaNO}_{3}\right)$ and barium containing chemicals $\left[\mathrm{BaCl}_{2}\right.$ and $\left.\mathrm{Ba}\left(\mathrm{NO}_{3}\right)_{2}\right]$, were used. As an osmotic control, mannitol was applied. Chemicals were applied at 200 and $300 \mathrm{mM}$ for divalent ion (calcium and barium) containing chemicals, 300 and $450 \mathrm{mM}$ for monovalent ion (sodium) containing chemicals, and 470 and $730 \mathrm{mM}$ for mannitol. The concentrations of calcium containing chemicals were determined based on the preliminary experiments. The values of osmotic potential of high and low concentrations of $\mathrm{CaCl}_{2}$ and $\mathrm{Ca}\left(\mathrm{NO}_{3}\right)_{2}$ were -1.3 and $-2.0 \mathrm{MPa}$, respectively. The osmotic 
potential of calcium containing chemicals were used to calculate the corresponding concentrations of other chemicals (Fig. 3.1). Osmotic potential measured with a dewpoint potentiometer (WP4-T, Decagon Devices Inc., Pullman, WA). Plants were drenched with chemical solutions $(60 \mathrm{~mL}$ per pot). Seven plants treated with each chemical had water withheld (water-stressed, $n=7$ ) until all treated plants reached a visual wilt status rating of 3 or below (unmarketable) as described by Waterland et al. (2010a). Three plants of each treatment were irrigated daily with $100 \mathrm{mg} \cdot \mathrm{L}^{-1} \mathrm{~N}$ (irrigated daily, $\mathrm{n}=3$ ) to determine whether treatments caused any side effect on plants.

Visual observations of wilt status were taken daily. Wilt status ratings were from 1 to 5 with $5=$ completely turgid, $4=$ soft to the touch but still upright, $3=$ starting to wilt, $2=$ severely wilted, and $1=$ wilted to the point that leaves are dried and desiccated (Waterland et al., 2010a). The shelf life of water-stressed plants was calculated as the number of days from the initiation of water being withheld until plants reached a wilt status rating of 3 (Waterland et al., 2010a).

Necrotic area percentage (NAP) was determined $5 \mathrm{~d}$ after osmotic treatment using the top view images of daily irrigated viola. Necrotic area percentage is a proportion of necrotic area to overall plant area on the image, which is measured by pixels of the green area of leaves and the overall plant leaf area. The image color was calibrated with a color calibration chart (ColorChecker Classic, Grand Rapids, MI) and image analysis was performed with Photoshop Creative Cloud 2018 (Adobe Systems, San Jose, CA). The NAP was calculated by the following equation: NAP $(\%)=100-[($ pixels of green leaf area) $/($ pixels of overall plant leaf area $) \times 100]$ (Wang et al., 2008).

Expt. 2: Wilt status, stomatal conductance, and relative water content of $\mathrm{CaCl}_{2}$-treated viola.

Seeds of V. cornuta 'Sorbet XP Yellow' were sown in 288-plug tray on Sept.10, 2018, and seedlings were transplanted into $11-\mathrm{cm}$ pots three weeks later and grown in the greenhouse as described previously. Average PPFD was $227 \mu \mathrm{mol} \cdot \mathrm{m}^{-2} \cdot \mathrm{s}^{-1}$ from 0600 to $2000 \mathrm{HR}$ daily (DLI = $\left.10.82 \mathrm{~mol} \cdot \mathrm{m}^{-2} \cdot \mathrm{d}^{-1}\right)$. Average greenhouse temperatures were $22.0 / 17.3 \pm 2.0 / 1.8^{\circ} \mathrm{C}$ day $/ \mathrm{night}$ (mean $\pm \mathrm{SD}$ ) with mean daytime relative humidity of $37.5 \%$. Based on the observations from Expt. 1, $\mathrm{CaCl}_{2}$ was selected for the additional experiment. The $\mathrm{CaCl}_{2}$ solutions (200 and 300 $\mathrm{mM})$ were separately drenched on 13 -week-old plants $(60 \mathrm{~mL}$ per pot). Plants were watered to container capacity 12 hours before application. Half of control and $\mathrm{CaCl}_{2}$-treated plants were irrigated $(n=5)$ and the other half were water-stressed $(n=5)$ as described above. After all 
water-stressed plants reached a wilt status rating of 3 or below, they were rewatered daily to determine if there was any damage by $\mathrm{CaCl}_{2}$ treatment. Visual wilt status of stressed plants was evaluated as described previously.

Stomatal conductance was measured with a portable photosynthesis system (LI-6400XT; LICOR, Lincoln, NE). Stomatal conductance measurements were taken one day before treatment, 4 hours after the treatment, daily until all plants showed visual wilting, and three days after plants were rewatered. A leaf was inserted into an extended chamber with clear top and bottom covers (Extended Reach $1 \mathrm{~cm}$ Chamber LI6400-15; LI-COR). Environmental conditions in the chamber were set at $400 \mu \mathrm{mol} \cdot \mathrm{m}^{-2} \cdot \mathrm{s}^{-1} \mathrm{CO}_{2}$, and $25^{\circ} \mathrm{C}$ as the block temperature. Readings were conducted from 1000 to $1400 \mathrm{HR}$. Leaf relative water content (RWC) was determined according to the following equation: RWC $(\%)=[(F W-D W) /(T W-D W)] \times 100($ Arndt et al., 2015). Fresh weight (FW) was measured immediately after leaf collection, and dry weight (DW) was measured after drying leaves in an oven for $72 \mathrm{~h}$ at $60{ }^{\circ} \mathrm{C}$. Turgid weight (TW) was determined after fresh leaf samples were placed in a $50 \mathrm{ml}$ tube with the cut end petiole submerged in distilled water for $24 \mathrm{~h}$ under light. Stomatal conductance and the leaf RWC were measured with three fully expanded leaves per plant, and the averaged value of three leaves per plant represented as one replication. Data are the means of measurements from five replications (or five plants).

\section{Statistical analysis}

Experiments were conducted as a randomized complete block design. Analysis of variance was performed by SAS (version 9.3; SAS Institute, Cary, NC). Plants were blocked by replication based on plant position in the greenhouse and watering regimen (irrigated daily vs. water-stressed). Difference among the treatment means were assessed by Tukey's test at $P \leq$ 0.05 .

\subsection{Results}

\section{Expt. 1: Effects of osmotic treatment on shelf life in Viola.}

To examine the effects of osmotic treatment on water deficit stress tolerance, calcium containing chemicals $\left[\mathrm{CaCl}_{2}\right.$ and $\left.\mathrm{Ca}\left(\mathrm{NO}_{3}\right)_{2}\right]$, sodium containing chemicals $\left(\mathrm{NaCl}, \mathrm{NaNO}_{3}\right)$, and barium containing chemicals $\left[\mathrm{BaCl}_{2}\right.$ and $\left.\mathrm{Ba}\left(\mathrm{NO}_{3}\right)_{2}\right]$ were selected in the experiment. Additionally, as an osmotic control, mannitol was applied. 
Among the seven chemicals, $300 \mathrm{mM} \mathrm{CaCl}_{2}, 300 \mathrm{mM} \mathrm{NaNO}_{3}, 200 \mathrm{mM} \mathrm{BaCl}_{2}$, and $200 \mathrm{mM}$ $\mathrm{Ba}\left(\mathrm{NO}_{3}\right)_{2}$ delayed wilting symptoms and extended shelf life by 1.3 to $1.9 \mathrm{~d}(P<0.0001)$, but other treatments showed no significant difference in shelf life compared to control (Table 3.1). Some treatments caused leaf necrosis, especially at the higher concentration of $\mathrm{NaCl}, \mathrm{NaNO}_{3}$, $\mathrm{BaCl}_{2}, \mathrm{Ba}\left(\mathrm{NO}_{3}\right)_{2}$, and mannitol (Table 3.1 and Fig. 3.2). Under water deficit, all treatments at the higher concentration, except the treatments with $\mathrm{Ca}$ containing chemicals, caused at least $70 \%$ of the plants to have leaf damage (Table 3.1 ) and 7.4 to $18.6 \%$ NAP on the continually irrigated plants (Table 3.1 and Fig. 3.2). Overall, the Ca containing chemicals extended shelf life the most without any significant leaf damage or necrosis. Of the two Ca containing chemicals, $\mathrm{CaCl}_{2}$ treatment exhibited no leaf damage at all and very little necrosis (Table 3.1). Therefore, $\mathrm{CaCl}_{2}$ treatment was chose for the further analysis.

Expt. 2: Wilt status, stomatal conductance, and relative water content of $\mathrm{CaCl}_{2}$-treated viola.

Calcium chloride treatment was performed to further examine the possible mode of action for enhanced water deficit tolerance. Wilt status and shelf life extension were re-examined. Additionally, relative water content (RWC) and stomatal conductance $(g \mathrm{~s})$ were measured. As in Expt. $1, \mathrm{CaCl}_{2}$ treatment was reconfirmed to enhance water deficit stress tolerance in viola

Stressed control plants started to wilt $2 \mathrm{~d}$ after treatment and reached a leaf desiccated status (wilt status rating of 1) $5 \mathrm{~d}$ after treatment, while leaves of $\mathrm{CaCl}_{2}$-treated plants remained turgid (above a wilt status rating of 3)(Fig. 3.3). Comparing the effects of two concentrations, plants treated with $200 \mathrm{mM} \mathrm{CaCl}_{2}$ had a higher wilt status rating than those with $300 \mathrm{mM}$ after 3 and 4 $\mathrm{d}$ of treatment $(P<0.0001)$ (Fig. 3.3). Four days after treatment, leaves treated with $200 \mathrm{mM}$ $\mathrm{CaCl}_{2}$ were the most turgid under water deficit condition, while control was severely wilted (Figs. 3.3 and 3.4). Furthermore, $\mathrm{CaCl}_{2}$ treatments doubled the shelf life compared to control ( $P$ $<0.001$ )(Figs. 3.3 and 3.4, Table 3.2), and $\mathrm{CaCl}_{2}$ at $200 \mathrm{mM}$ had longer shelf life than at 300 $\mathrm{mM}$ by $1.4 \mathrm{~d}$ (Table 3.2).

To examine whether osmotic pressure caused stomatal closure, $g$ s was measured. Stomatal conductance rapidly decreased compared with that of control plants within $4 \mathrm{~h}$ after $\mathrm{CaCl}_{2}$ treatment in both irrigated and water-stressed viola (Fig. 3.5A and B). As water stress progressed, $g \mathrm{~s}$ of stressed control plants became similar to that of $\mathrm{CaCl}_{2}$-treated plants $3 \mathrm{~d}$ after treatment (Fig. 3.5B). The reduced $g \mathrm{~s}$ in $\mathrm{CaCl}_{2}$-treated and water-stressed viola remained low after resuming irrigation for $3 \mathrm{~d}$ (Fig. 3.5B). There is no difference between high and low $\mathrm{CaCl}_{2}$ 
concentrations in $g \mathrm{~s}$ under water deficit and after water resumed (Fig. 3.5B). However, $g \mathrm{~s}$ of stressed control was not measurable because they were severely desiccated after $5 \mathrm{~d}$ of water deficit (Fig. 3.5B). In contrast to the water-stressed plants, when plants were irrigated daily, $g \mathrm{~s}$ of $\mathrm{CaCl}_{2}$-treated plants was gradually increased $3 \mathrm{~d}$ after treatment and regained the level of $g \mathrm{~s}$ similar to that of irrigated controls $7 \mathrm{~d}$ after treatment (Fig. 3.5A). During the irrigation, a faster increase of $g \mathrm{~s}$ was observed with the $200 \mathrm{mM} \mathrm{CaCl}_{2}$ than $300 \mathrm{mM} \mathrm{CaCl}_{2}$ (Fig. 3.5A).

When plants were irrigated daily, both controls and $\mathrm{CaCl}_{2}$-treated plants maintained high leaf RWC, ranging 84-97\% during the experiment (Fig. 3.5C). After 1 and $2 \mathrm{~d}$ of water deficit, leaf RWC of plants treated with $\mathrm{CaCl}_{2}$ was reduced to $83-89 \%$, while stressed controls maintained leaf RWC above $92 \%$ (Fig. 3.5D). However, the leaf RWC of stressed controls were dramatically reduced $3 \mathrm{~d}$ after treatment and continued to decrease to $50 \%$ after $4 \mathrm{~d}$ of treatment (Fig. 3.5D) In contrast, the leaves of $\mathrm{CaCl}_{2}$-treated plants maintained leaf $\mathrm{RWC}$ above $70 \%$ during the water deficit stress (Fig. 3.5D). Leaf RWC of plants treated with $200 \mathrm{mM} \mathrm{CaCl}_{2}$ was generally higher than those treated with $300 \mathrm{mM} \mathrm{CaCl}_{2}$ after $4 \mathrm{~d}$ of water deficit until the end of rewatering period (Fig. 3.5D). Leaf RWC of the stressed control plants was not measured after 4 days due to the desiccation of the plants (Fig. 3.5D).

\subsection{Discussion}

Water deficit stress is one of the major threats to postproduction decline in the floriculture industry. To enhance temporary water deficit tolerance, osmotic treatments with seven chemicals $\left[\mathrm{CaCl}_{2}, \mathrm{Ca}\left(\mathrm{NO}_{3}\right)_{2}, \mathrm{NaCl}, \mathrm{NaNO}_{3}, \mathrm{BaCl}_{2}, \mathrm{Ba}\left(\mathrm{NO}_{3}\right)_{2}\right.$, and mannitol] were evaluated in viola. Chemicals were applied at two concentrations whose osmotic potential was -1.3 and -2.0 MPa, respectively (Fig. 3.1). The applications of $\mathrm{CaCl}_{2}, \mathrm{NaNO}_{3}, \mathrm{BaCl}_{2}$, and $\mathrm{Ba}\left(\mathrm{NO}_{3}\right)_{2}$ were effective in delaying plant wilting (Table 3.1). However, leaf necrosis was observed in plants treated with $\mathrm{NaCl}, \mathrm{NaNO}_{3}, \mathrm{BaCl}_{2}, \mathrm{Ba}\left(\mathrm{NO}_{3}\right)_{2}$, and mannitol (Table 3.1 and Fig. 3.2). Treatment of $\mathrm{CaCl}_{2}$ induced stomatal closure and limited transpirational water loss, consequently delaying wilting symptoms (Figs. 3.3-3.5 and Table 3.2).

Generally, osmotic treatments extended shelf life of violas compared to control treatment (Table 3.1), indicating that chemical-induced osmotic stress might have caused plants to trigger a response mechanism to temporarily tolerate water deficit stress. Despite the positive effect, sodium and barium containing chemicals and mannitol caused severe leaf necrosis and their 
aesthetic quality was diminished (Table 3.1 and Fig. 3.2). Low level of $\mathrm{Na}^{+}$is beneficial in many crops, but the excess uptake of $\mathrm{Na}^{+}$can negatively affect cellular biochemistry, leading to plant death (Maathuis, 2013). All barium treatments resulted in leaf necrosis, indicating that barium containing chemicals between 200 and $300 \mathrm{mM}$ were not appropriate agents for violas, although they increased shelf life at the lower concentration. Suwa et al. (2008) reported that barium treatments at 100,1000 , and $5000 \mu \mathrm{M}$ induced phytotoxicity, inhibited photosynthetic activity, and reduced crop production in soybean (Glycine max) under hydroponic system. Negative effects of mannitol application have been often reported in many studies (Adrees et al., 2015; Fazal et al., 2014; Sassi et al., 2010; Slama et al., 2007). Fazal et al. (2014) reported that mannitol application $(137 \mathrm{mM})$ reduced shoot and root length and biomass of canola (Brassica napus), cauliflower (Brassica oleracea), and tomato (Solanum lycopersicum) seedlings. Mannitol, used as an osmotic inducing agent in this study, appeared to fail to enhance tolerance to water deficit stress due to tissue damages. It is possible that a lower concentration of $\mathrm{Na}, \mathrm{Ba}$ containing chemicals and mannitol do not cause side effects; however, the concentrations whose osmotic potential was higher than -1.3 MPa did not extend shelf life in the preliminary experiments. Comparing to $\mathrm{CaCl}_{2}, \mathrm{Ca}\left(\mathrm{NO}_{3}\right)_{2}$ did not extend shelf life at both concentrations (Table 3.1). Chloride $\left(\mathrm{Cl}^{-}\right)$is an essential plant nutrient, but the accumulation of $\mathrm{Cl}^{-}$is a significant contributor in salt stress (Li et al., 2017a). Geilfus et al. (2015) reported that exposure of $\mathrm{Cl}^{-}$induced stomata closure by modulating the ABA redistribution in Vicia faba, suggesting a salinity stressor, chloride, causes ABA-dependent stomatal closure to save water under salinity stress. Therefore, chloride from $\mathrm{CaCl}_{2}$ treatment in addition to $\mathrm{Ca}$ might have attributed to inducing stomatal closure and stress mechanisms in this study.

The treatment of $\mathrm{CaCl}_{2}$ showed a sharp reduction of $g \mathrm{~s}$ within $4 \mathrm{~h}$ after treatment and a slight reduction of leaf RWC compared to control plants during the water deficit period (Fig. 3.5). Stomatal closure helps plants to prevent water loss. For example, an application of antitranspirants, chemicals designed to physically block stomata, improved tolerance to water deficit stress (Park et al., 2016). Furthermore, as observed in $\mathrm{CaCl}_{2}$-treated plants irrigated daily, $g$ s was declined rapidly and then gradually increased to the same level of irrigated controls, suggesting the high concentration of $\mathrm{CaCl}_{2}$ caused stomatal closure immediately after the application (Fig. 3.5). Therefore, an application of high concentration of $\mathrm{CaCl}_{2}$ could have induced an osmotic stress, and $\mathrm{CaCl}_{2}$-induced osmotic stress could have triggered a mechanism 
to rapidly close stomata. The subsequent reduction of transpirational water loss might have delayed plant wilting resulting in enhanced shelf life. Additionally, calcium appeared to alleviate the adverse effects of water deficit stress in this study. Calcium acts as a secondary messenger regulating stress mechanisms that help plants adjust to adverse environments (Ranty et al., 2016). Exogenous calcium treatments have been observed to enhance tolerance in several crops under different abiotic stresses by regulating plant cell metabolism (Shoresh et al., 2011; Tan et al., 2011; Upadhyaya et al., 2011; Xu et al., 2013a). The spray treatment of $\mathrm{CaCl}_{2}$ enhanced water deficit tolerance by modulating antioxidant enzyme activities, maintaining the integrity of cell membrane, and increasing photosynthetic efficiency in Zoysia japonica (Xu et al., 2013a). $\mathrm{CaCl}_{2}$ treatment also alleviated water deficit-induced damages and increased photosynthetic performance, such as a high chlorophyll content, in Vernicia fordii (Li et al., 2017c). Increases in osmoprotectants (proline, glycine betaine, and total free amino acids) were observed with calcium treatments in Triticum aestivum (Nayyar, 2003), Zea mays (Naeem et al., 2017; Nayyar, 2003), and Catharanthus roseus (Jaleel et al., 2007), suggesting exogenous $\mathrm{Ca}^{2+}$ contributes osmotic adjustment under water deficit. In addition to potential osmotic effect of high concentration of $\mathrm{CaCl}_{2}$, calcium might have contributed to enhancing tolerance as a signal to trigger a responsive mechanism to water deficit stress at the cellular level.

Even though treatment of $\mathrm{CaCl}_{2}$ at 200 and $300 \mathrm{mM}$ were effective in delaying plant wilting and enhancing water deficit tolerance, higher concentration would be detrimental for plant growth and development. Stomatal conductance of $\mathrm{CaCl}_{2}$-treated plants remained low for at least 2 days after rewatering (Fig. 3.5A). Under well-watered condition, $g \mathrm{~s}$ of $\mathrm{CaCl}_{2}$-treated plants was significantly lower than that of control until $4 \mathrm{~d}$ after treatment (Fig. 3.5A). This result indicated that osmotic treatment with higher concentration of $\mathrm{CaCl}_{2}$ maintained stomata closed longer and can be accompanied by compromised plant growth and quality. Prolonged stomatal closure can increase heat stress under high temperatures, reduce photosynthesis, and may cause phototoxicity and necrosis (Kim and van Iersel, 2011; Waterland et al., 2010a; Waterland et al., 2010c). However, no side effect was observed in this study. In addition, high concentration could reduce the efficacy of $\mathrm{CaCl}_{2}$ treatment on increasing water deficit tolerance. While the treatment with $300 \mathrm{mM} \mathrm{CaCl}_{2}$ showed the longest shelf life in Expt. 1, $\mathrm{CaCl}_{2} 300 \mathrm{mM}$ had shorter shelf life than $\mathrm{CaCl}_{2} 200 \mathrm{mM}$ in Expt. 2 (Tables 3.1 and 3.2). Although $\mathrm{CaCl}_{2} 300 \mathrm{mM}$ delayed plant wilting compared to control by inducing stomatal closure, osmotic stress by the high 
concentration might have hastened plant wilting. The efficacy of high concentration might be dependent on plant stage, size, and/or environmental conditions. Indeed, the light intensity were slightly higher in Expt. 2.

The effects of osmotic treatment were evaluated to increase temporary water deficit stress tolerance in viola. Among seven chemicals used in this study, $\mathrm{CaCl}_{2}$ treatment was the most effective in enhancing shelf life without any observable side effect. Osmotic treatments could be utilized to help reduce bedding crop loss due to water deficit stress during postproduction period. However, caution should be applied to prevent any side effects due to the toxic effect at high concentration and potential damage caused by prolonged stomatal closure. Also, the efficacy of osmotic treatment could be species-dependent. Further research is needed to evaluate the feasibility of utilizing economically viable chemicals and fertilizer application at high concentration prior to shipping as well as to assess any side effect caused by such applications. 


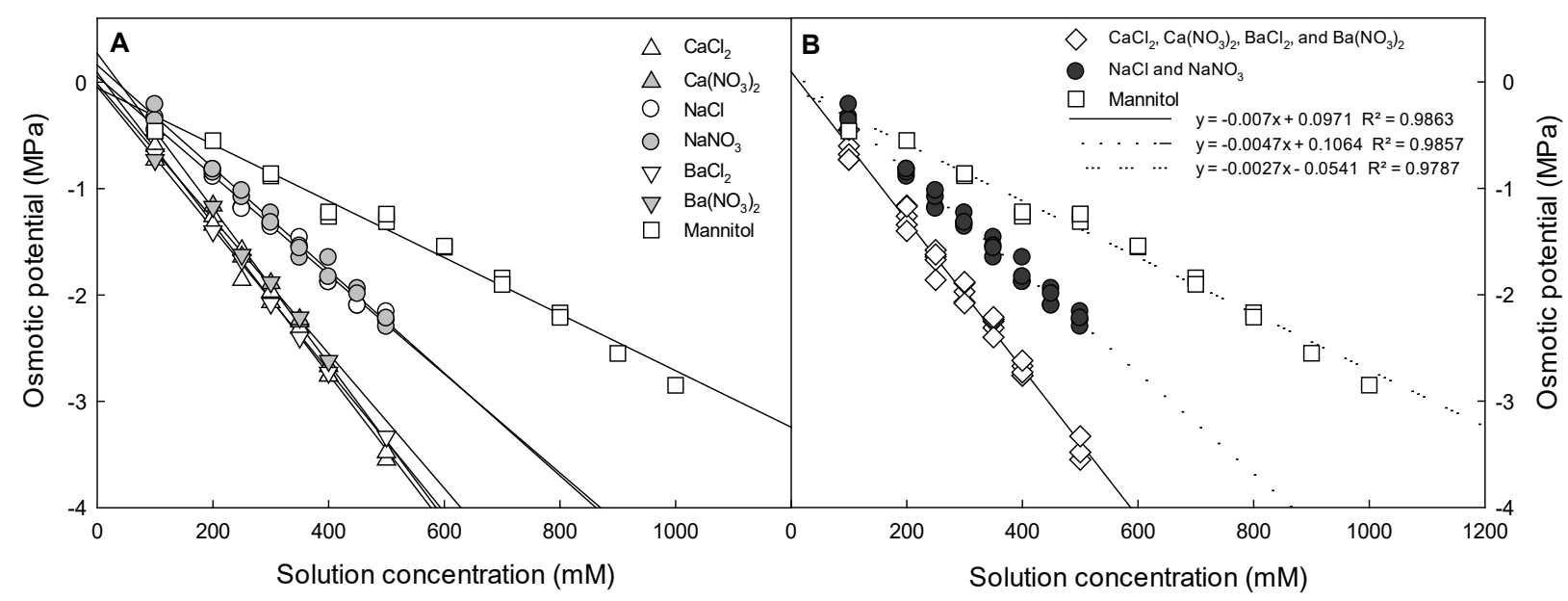

Fig. 3.1. Osmotic potential of chemical solutions measured with a dewpoint potentiometer. The left graph (A) shows regression lines of each solution, and the right graph (B) shows three regression lines of divalent ion containing chemicals $(\mathrm{Ca}$ and $\mathrm{Ba})$, monovalent ion containing chemicals $(\mathrm{Na})$, and mannitol alone. The regression equations obtained from the right graph were used to calculate the chemical application concentration for each chemical. 
Table 3.1. Shelf life, percentage of damaged plants, and necrotic area percentage (NAP) of viola treated with chemicals.

\begin{tabular}{lcccc}
\hline Chemical & Conc. $(\mathrm{mM})$ & $\begin{array}{c}\text { Shelf life } \\
(\mathrm{d})^{\mathrm{z}}\end{array}$ & $\begin{array}{c}\text { Percentage of } \\
\text { damaged plants }(\%)^{\mathrm{y}}\end{array}$ & NAP $(\%)^{\mathrm{x}}$ \\
\hline $\mathrm{Control}$ & 0 & $2.4 \mathrm{bc}^{\mathrm{w}}$ & 0 & $0.0 \mathrm{a}$ \\
$\mathrm{CaCl}_{2}$ & 200 & $3.6 \mathrm{ab}$ & 0 & $0.0 \mathrm{a}$ \\
& 300 & $4.3 \mathrm{a}$ & 0 & $0.4 \mathrm{a}$ \\
$\mathrm{Ca}\left(\mathrm{NO}_{3}\right)_{2}$ & 200 & $3.6 \mathrm{ab}$ & 0 & $0.0 \mathrm{a}$ \\
& 300 & $3.4 \mathrm{ab}$ & 14.3 & $1.1 \mathrm{a}$ \\
$\mathrm{NaCl}$ & 300 & $3.6 \mathrm{ab}$ & 0 & $0.0 \mathrm{a}$ \\
& 450 & $3.4 \mathrm{ab}$ & 85.7 & $13.9 \mathrm{a}$ \\
$\mathrm{NaNO}_{3}$ & 300 & $4.0 \mathrm{a}$ & 0 & $0.0 \mathrm{a}$ \\
& 450 & $3.0 \mathrm{abc}$ & 100 & $7.4 \mathrm{a}$ \\
$\mathrm{BaCl}$ & 200 & $4.0 \mathrm{a}$ & 14.3 & $12.1 \mathrm{a}$ \\
& 300 & $3.0 \mathrm{abc}$ & 100 & $10.7 \mathrm{a}$ \\
$\mathrm{Ba}_{2}\left(\mathrm{NO}_{3}\right)_{2}$ & 200 & $3.7 \mathrm{a}$ & 42.9 & $10.7 \mathrm{a}$ \\
& 300 & $3.0 \mathrm{abc}$ & 71.4 & $18.6 \mathrm{a}$ \\
$\mathrm{Mannitol}$ & 470 & $3.3 \mathrm{abc}$ & 28.6 & $8.1 \mathrm{a}$ \\
& 730 & $2.0 \mathrm{c}$ & 100 & $17.8 \mathrm{a}$ \\
\hline Significance & & $* * *$ & N/A & $* *$ \\
\hline
\end{tabular}

${ }^{\mathrm{z}}$ The number of days from the initiation of water being withheld until plants started showing visible symptoms of wilting. Values are means of seven replications $(n=7)$.

${ }^{\mathrm{y}}$ The percentage of damaged plants out of seven treated plants under water deficit stress.

${ }^{\mathrm{x}}$ The proportion of necrotic area to overall plant area, which is measured by pixels of the green leaf area and the overall plant leaf area. The top view images of treated violas with daily irrigation for 5 days were used. NAP $(\%)=$ $100-[($ pixels of green leaf area) / (pixels of overall plant leaf area $) \times 100]$.

Values are means of three replications $(n=3)$.

${ }^{\text {w }}$ Mean separation by Tukey's significance test at $P \leq 0.05$.

**,*** Significant at $P \leq 0.01$ or 0.001 , respectively.

${ }^{\mathrm{N} / \mathrm{A}}$ Not applicable. 


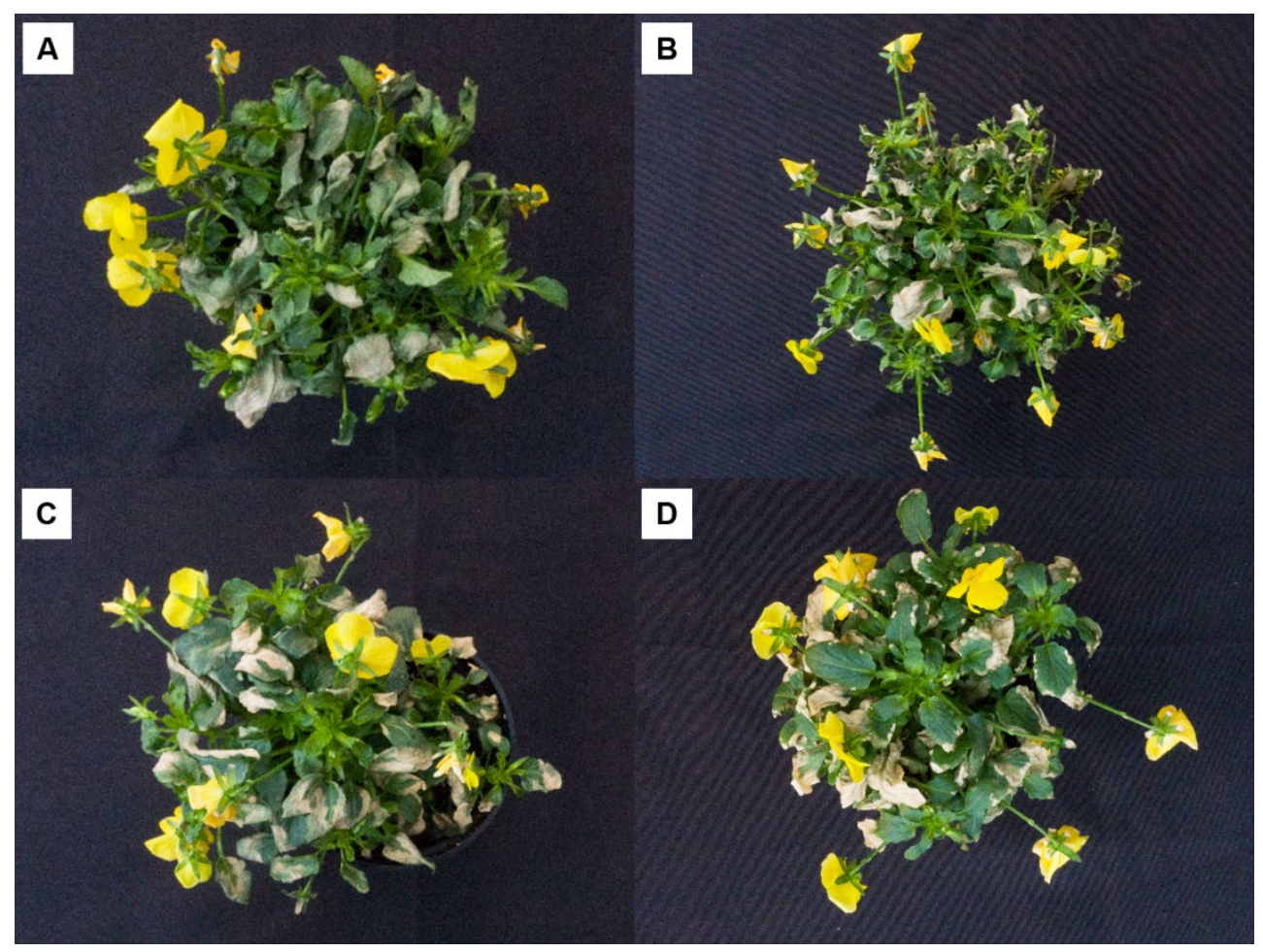

Fig. 3.2. Foliage damage of viola treated by $\mathrm{NaCl}$ at $450 \mathrm{mM}(\mathrm{A}), \mathrm{NaNO}_{3}$ at $450 \mathrm{mM}$ (B), $\mathrm{Ba}\left(\mathrm{NO}_{3}\right)_{2}$ at $300 \mathrm{mM}(\mathrm{C})$, and mannitol at $730 \mathrm{mM}(\mathrm{D})$ with daily irrigation. Images were taken at 5 days after osmotic treatments. 


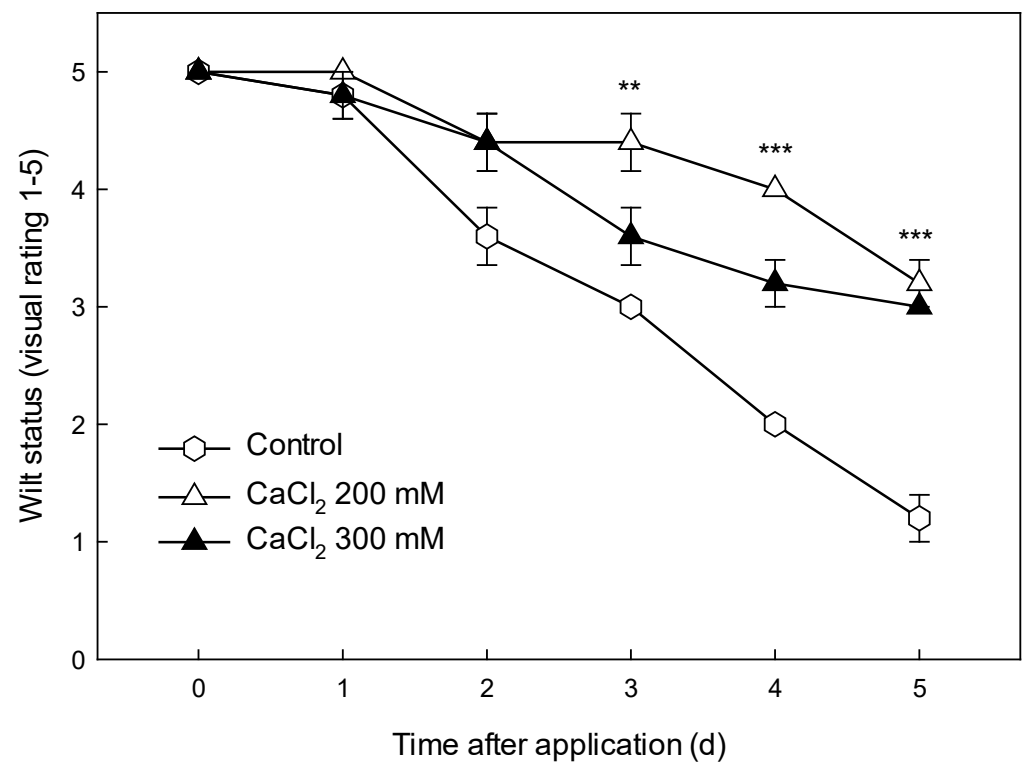

Fig. 3.3 Daily wilt status rating of viola treated with $\mathrm{CaCl}_{2}$ at 200 and $300 \mathrm{mM}$ under water deficit stress. Wilt status ratings were from 5 to 1 , where $5=$ completely turgid, $4=$ soft to touch but still upright, $3=$ starting to wilt, $2=$ severely wilted, and $1=$ wilted to the point that leaves are desiccated (Waterland et al., 2010a) Vertical bars are standard error of the means with five replications $(\mathrm{n}=5) .{ }^{* * * * * *}$ Significant at $P \leq 0.01$ or 0.001 , respectively. 


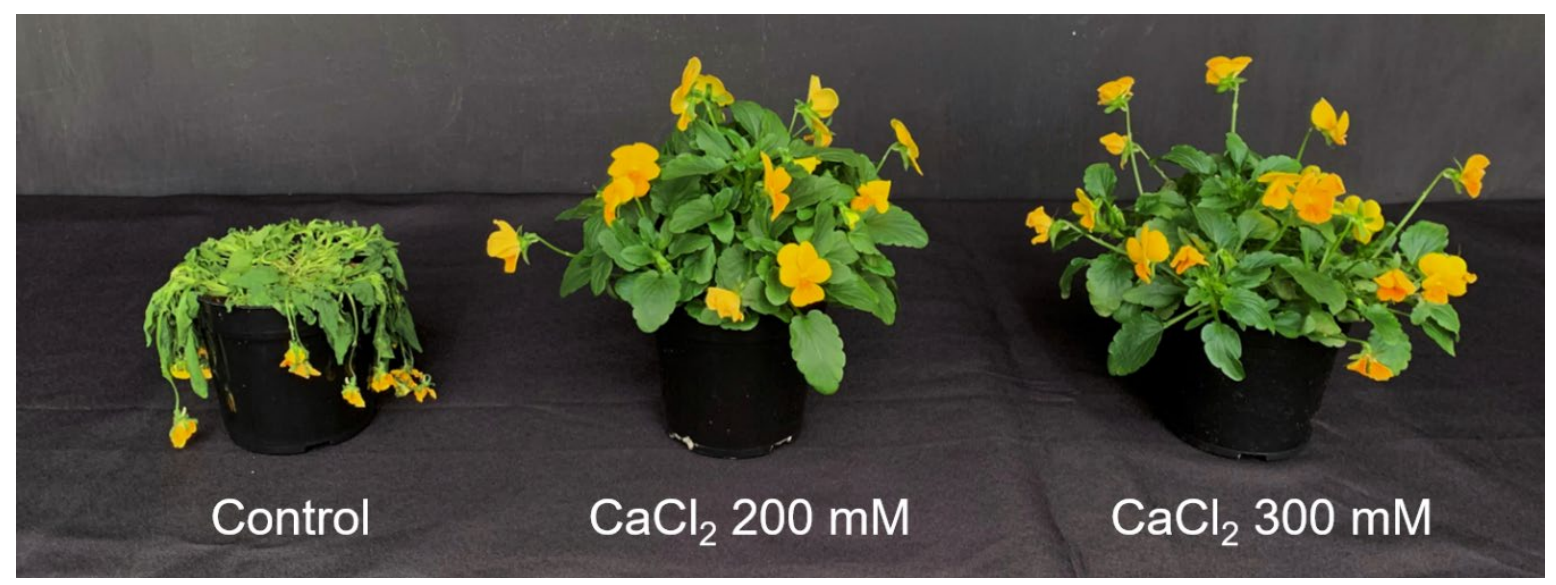

Fig. 3.4. Viola treated with control, $\mathrm{CaCl}_{2}$ at 200 and $300 \mathrm{mM}$ (from left to right). Plants were water deficit-stressed until $\mathrm{CaCl}_{2}$-treated plants began to wilt. The image was taken at 4 days after application of $\mathrm{CaCl}_{2}$ and water deficit. 
Table 3.2. Shelf life of $\mathrm{CaCl}_{2}$-treated viola under water deficit.

\begin{tabular}{lcc}
\hline Chemical & Conc. $(\mathrm{mM})$ & Shelf life $(\mathrm{d})^{\mathrm{z}}$ \\
\hline Control & 0 & $2.6 \mathrm{c}^{\mathrm{y}}$ \\
$\mathrm{CaCl}_{2}$ & 200 & $5.2 \mathrm{~A}$ \\
& 300 & $3.8 \mathrm{~B}$ \\
\hline Significance & $* * *$ \\
\hline${ }^{\mathrm{z}}$ The number of days from the initiation of water being \\
withheld until plants started showing visible symptoms of \\
wilting. Values are means of five replications $(\mathrm{n}=5)$. \\
${ }^{\mathrm{y}}$ Mean separation by Tukey's significance test at $P \leq 0.05$. \\
${ }^{* * *}$ Significant at $P \leq 0.001$.
\end{tabular}




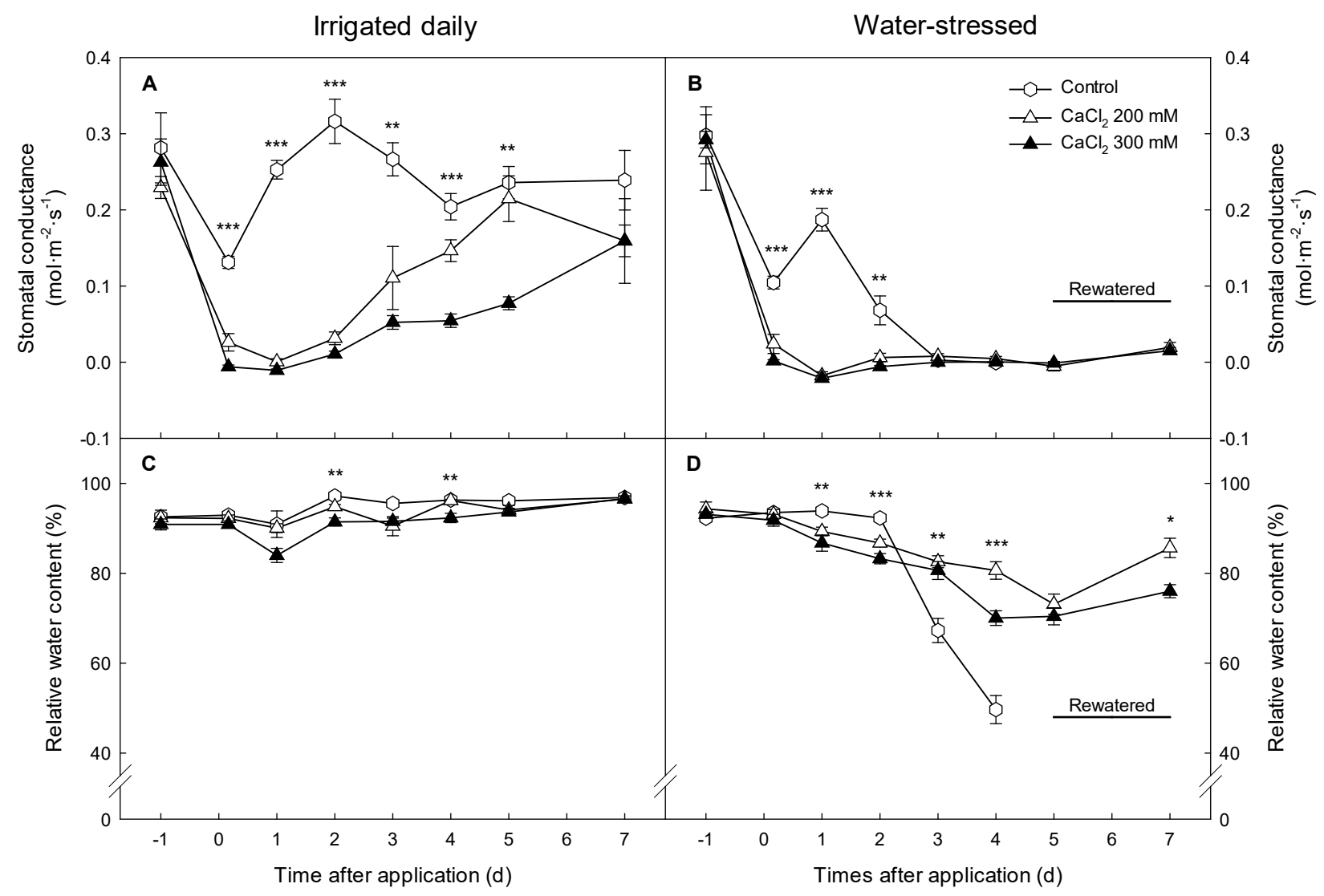

Fig. 3.5. Stomatal conductance ( $g \mathrm{~s}, \mathrm{~A}$ and B) and leaf relative water content (RWC, C and D) of $\mathrm{CaCl}_{2}$-treated viola. Half of plants were irrigated daily (A and $\mathrm{C}$ ), and the other half had water withheld until plants had visual wilting symptoms and irrigation was resumed for 3 days (B and D). Measurements were performed 1 day before the application, 4 hours after the application, daily until all plants showed wilting symptoms, and 3 days after plants were rewatered. Stressed control was not measurable 5 days after water deficit since they were severely desiccated due to water deficit stress (B and D). Vertical bars are standard errors of the means with five replications $(\mathrm{n}=5) .{ }^{*},{ }^{* *},{ }^{* * *}$ Significant at $P \leq 0.05,0.01$, or 0.001 , respectively. 


\section{Chapter 4 Time-course transcriptomic analysis of Petunia $\times$ hybrida leaves under water deficit stress using RNA sequencing}

\subsection{Abstract}

Water deficit limits plant growth and development, resulting in quality loss of horticultural crops. However, there is limited information on gene regulation and signaling pathways related to water deficit stress response examined at multiple time points in petunia. The objective of this research was to investigate global gene expression patterns under water deficit stress to provide an insight into how petunia (Petunia $\times$ hybrida 'Mitchell Diploid') responded in the process of stress. Nine-week-old petunias were irrigated daily or placed under water stress by withholding water. Stressed plants reduced stomatal conductance after five days of water deficit, indicating they perceived stress and initiated stress response mechanisms within five days. To analyze transcriptomic changes at the early stage of water deficit, leaf tissue samples were collected 1, 3, and 5 days after water was withheld. Time-course transcriptomic profiling under water stress were examined using RNA sequencing. Nearly 270 million reads were sequenced by Illumina HiSeq 1500, and de novo assembly using Trinity software generated 76,601 contigs. Then, 72,474 nonredundant transcripts were obtained by CD-HIT-EST. Under water deficit stress, 154, 3611, and 980 genes were upregulated and 41, 2806, and 253 genes were downregulated on day 1,3 , and 5, respectively. RNA sequencing data were confirmed by qPCR analysis with five genes showing distinct expression patterns over three time points $\left(\mathrm{R}^{2}=0.94\right)$. Gene Ontology and Kyoto Encyclopedia of Genes and Genomes analyses revealed that redox homeostasis processes with sulfur metabolism and hormone signal transduction, especially abscisic acid and ethylene, were enriched under water deficit stress. Thirty-four transcription factor families were identified in petunia, including members of APETALA 2/ethylene response factor (AP2/ERF), no apical meristem, Arabidopsis thaliana activating factor, and cup-shaped cotyledon (NAC), myeloblastosis-related (MYB-related), $\mathrm{Cys}_{2}-\mathrm{His}_{2}$-like fold group (C2H2), and basic leucine zipper (bZIP) families. The transcriptome data from this research will provide valuable molecular resources for understanding the early stages of water stress-responsive networks as well as engineering petunia with enhanced water stress tolerance. 


\subsection{Introduction}

Faced with water scarcity, drought is one of the critical issues in the world. Climate change is predicted to cause more severe droughts in the future, aggravating the shortage of water resources. As water supplies are not able to meet water demands, regulation of water use becomes inevitable in agriculture. Water deficiency is a major constraint to crop productivity worldwide, thus leading to substantial crop loss and ultimately presenting issues of food insecurity (Farooq et al., 2009).

Ornamental horticulture has become an important portion in the horticultural industry. A variety of ornamental plants are widely used in home gardening, landscaping, and the cut flower industry as well (USDA, 2019). The wholesale value for all aspects of floriculture is estimated to be more than $\$ 4.63$ billion, and bedding plants make up about $47 \%$ of the total value of the ornamental plant market for 2018 in United States (USDA, 2019). Ornamental plants are produced at a premium quality, but their quality often declines during shipping and retailing without appropriate temperatures and irrigation. These adverse environments accelerate substrate drying and plant wilting. Crop losses due to these poor postproduction conditions are estimated to result in $5 \%$ to $20 \%$ of unsalable crops (Healy, 2009). Therefore, cultivars tolerant to water stress are needed to minimize the decline of crop quality and crop loss during shipping, retailing and the loss of crops at the final destinations. Better understanding of response mechanisms to water stress would provide valuable information for improvement of water stress tolerance in ornamental crops.

Plants have a wide range of morphological, physiological, and biochemical processes to cope with water deficit stress. These processes include development of large and deep root systems, stomatal closure, accumulation of osmolytes, antioxidant activity, and metabolism and signaling of phytohormones (Li et al., 2015; Liu et al., 2016). Various genes are involved in these stress responses, suggesting water deficit stress tolerance is mediated by more complex mechanisms than previously thought ( $\mathrm{Li}$ et al., 2016). It has been suggested that stress-responsive genes can be classified into two groups (Hadiarto and Tran, 2011). The first group of genes plays a direct role in stress tolerance, including enzymes involved in osmotic adjustment, antioxidant proteins, ion transporters, detoxification enzymes, chaperones, late embryogenesis abundant proteins, and various proteases (Dudhate et al., 2018). The second group includes the regulatory genes encoding protein kinases, protein phosphatases, and transcription factors (TFs) that mediate 
signal transduction pathways and transcription of downstream target genes (Hadiarto and Tran, 2011).

Despite considerable efforts to understand the water stress response pathways, comprehensive information on genetic regulatory networks and tolerance under water deficit still remain elusive. While Arabidopsis has been extensively investigated, it cannot represent all other extant species (Gerats and Vandenbussche, 2005). Furthermore, it has been suggested that molecular mechanisms of water stress response may differ between Arabidopsis and other plants, although some water deficit stress response pathways may be conserved (Li et al., 2016). Therefore, it is important to investigate the crop of interest so that molecular mechanisms specific to that plant may be identified.

Petunia $(P . \times$ hybrida) was investigated in this study because it is one of the most important ornamental crops used worldwide in landscaping. In addition, the genus Petunia is a member of the Solanaceae family along with tobacco, tomato, and pepper, and it represents a more diverse range of plants than Arabidopsis (Gerats and Vandenbussche, 2005). For several decades, petunia research had focused on flavonoid synthesis, floral development and senescence, but few studies regarding abiotic stress response at the molecular level have been conducted. Two zinc finger TFs (ZPT2-2 and ZPT2-3) have been studied for their roles in dehydration, wounding, and cold (Sugano et al., 2003). However, it is expected that various genes are involved in abiotic stress responses. In order to improve water deficit tolerance in petunia, it is desired to understand genome-wide expression profiling in responding to water deficit.

With rapid advances in next-generation sequencing, RNA sequencing (RNA-seq) has broadened the scope of gene expression studies from a few genes to the whole genome and from a limited number of model plants to a vast range of non-model species. While microarray requires previous genome annotation and pre-synthesized nucleotide probes, RNA-seq can be used to analyze the transcriptome of organisms in the absence of a reference genome and to discover novel genes (Xu et al., 2013b). Additionally, RNA-seq can provide more accurate qualitative and quantitative information of gene expression than the microarray-based assays (Yates et al., 2014). Because of these advantages, RNA-seq has been widely used to sequence transcripts and profile global expression data in popular (Barghini et al., 2015), pear (Li et al., 2016), wild rice (Tian et al., 2015), maize (Li et al., 2017b), and Chinese cabbage (Eom et al., 
2018) under abiotic stresses. However, to the best of my knowledge, no research has been performed on the transcriptomic profiling under water deficit in petunia using RNA-seq.

In this study, RNA-seq was performed to generate time-course transcriptome data with petunia. The specific research objectives were to 1) perform and analyze transcriptome to provide a global gene expression profile at three time point of water deficit, 2) identify differentially expressed genes (DEGs), and 3) determine the main biological processes involved in water deficit stress, and 4) identify TFs associated with water stress tolerance in petunia. The time-course transcriptomic data obtained from this project will provide valuable insight into molecular mechanisms underlying the early stage of water deficit stress responses and can ultimately be utilized to generate plants with enhanced water stress tolerant traits through breeding and genetic engineering.

\subsection{Materials and methods}

\section{Plant material, water deficit treatment, and tissue collection}

Seeds of $P$. $\times$ hybrida 'Mitchell Diploid' were sown in a 288-plug tray. Seedlings were transplanted into 11-cm pots with soilless media (Sunshine ${ }^{\circledR}$ Mix \#1; Sun Gro Horticulture, Agawam, MA, USA). Plants were grown under natural irradiation with supplemental lighting provided as needed by high-pressure sodium lighting (600W HS200 deep reflector, Hortilux, Pijnacker, The Netherlands) in a greenhouse (Morgantown, WV, USA). Plants were fertilized with Peter ${ }^{\circledR}$ Excel Cal-Mag 15N-2.2P-12.5K (Everris, Marysville, OH, USA) at $200 \mathrm{mg} \mathrm{N} \cdot \mathrm{L}^{-1}$, which was reduced to $100 \mathrm{mg} \mathrm{N} \cdot \mathrm{L}^{-1}$ one week before water deficit treatment. Nine-week-old petunias were randomly divided into two groups: control (C) and water-stressed (S). Plants in the control group were irrigated daily with $100 \mathrm{mg} \mathrm{N} \cdot \mathrm{L}^{-1}$ until the end of experiment, while the water deficit stress group received no water during water treatment up to five days. Stomatal conductance was measured, and leaf tissues were collected on 1st, 3rd and 5th day of water treatment with three replications $(\mathrm{n}=3)$. A fully expanded leaf (the seventh or eighth leaf from the top) per plant was collected. Instead of using control tissues collected at one time, control samples were collected together with stressed plants at the corresponding time to account for any circadian and/or environment effect at each time point. 


\section{Measurement of stomatal conductance}

Prior to leaf collection, stomatal conductance of three leaves per plant was measured between 10 am to 2 pm with a portable photosynthesis system (LI-6400XT; LI-COR Inc., Lincoln, NE, USA). A leaf was clamped into an extended chamber with clear top and bottom covers (Extended Reach $1 \mathrm{~cm}$ Chamber LI6400-15; LI-COR). Environmental conditions in the chamber were set at $400 \mu \mathrm{mol} \cdot \mathrm{m}^{-2} \cdot \mathrm{s}^{-1} \mathrm{CO}_{2}$, and $25^{\circ} \mathrm{C}$ as the block temperature. Readings were conducted from 1000 to $1400 \mathrm{HR}$. Data of stomatal conductance are the means of measurements from three plants $(\mathrm{n}=3)$. Reduced stomatal conductance is an indicator of plant response to water deficit stress. Analysis of variance (ANOVA) was performed by SAS 9.3 (SAS Institute, Inc., Cary, $\mathrm{NC})$.

\section{Total RNA extraction, RNA-seq library construction, and sequencing}

For RNA-seq analysis, leaf samples were collected one, three, and five days after withholding water from control and water-stressed plants, named herein $\mathrm{C} 1, \mathrm{C} 3, \mathrm{C} 5, \mathrm{~S} 1, \mathrm{~S} 3$, and $\mathrm{S} 5$, respectively. Three biological replicates were used $(\mathrm{n}=3)$. Total RNA was extracted using Trizol Reagent (Invitrogen, USA), and RNA was purified by phenol/chloroform extraction following the standard procedures. Total RNA quality was evaluated by an Agilent 2100 Bioanalyzer (Agilent, Santa Clara, CA, USA), with the average RNA integrity number of 7.54. cDNA libraries for sequencing were prepared using the TrueSeq RNA Sample Prep Kit according to the manufacturer's instructions (Illumina Inc., San Diego, CA, USA). These libraries were sequenced on an Illumina HiSeq1500 (Illumina Inc., San Diego, CA, USA), and reads were generated in 50bp paired-end format.

Data processing, de novo assembly, and annotation

Raw reads in FASTQ format were subjected to sequence quality control using FastQC (http://www.bioinformatics.babraham.ac.uk/projects/fastqc). Trimmomatic (http://www.usadellab.org/cms/?page=trimmomatic) was used to remove adapters and filter/trim the low-quality score reads if $\mathrm{Q}$ is less than 25 . Trimmed reads with minimum length of $40 \mathrm{bp}$ were de novo assembled into contigs using Trinity (version 2.2.0)(Haas et al., 2013). De novo assembly was used since a reference genome for $P$. $\times$ hybrida was not available. After removing contigs shorter than $200 \mathrm{bp}$, CD-HIT-EST was used to cluster sequences with $90 \%$ identical contigs/isoforms to obtain nonredundant transcripts (Niu et al., 2010). To perform functional annotation, all transcripts were compared to Solanum lycopersicum protein database 
(ftp://ftp.ncbi.nlm.nih.gov/genomes/Solanum_lycopersicum/protein/) with cut-off E-value of $1 \times$ $10^{-5}$ using BLASTx (Altschul et al., 1997). The resulting BLAST results were fed into Blast2GO (http://www.blast2go.com) to predict Gene Ontology (GO) annotation, describing biological processes, molecular functions, and cellular component (Conesa and Götz, 2008). Web Gene Ontology Annotation Plot (WEGO) was used to visualize GO categories (Zheng et al., 2006).

Gene expression quantification and differential expression analysis

The RNA-seq reads were aligned to the assembled transcripts using Bowtie2 (http://bowtiebio.sourceforge.net/bowtie2)(Langmead and Salzberg, 2012), and transcript abundance was assessed by RNA-seq By Expectation Maximization (RSEM) software (http://deweylab.biostat.wisc.edu/resm)(Li and Dewey, 2011) bundled with the Trinity package (Haas et al., 2013). The expression levels were normalized with Trimmed Mean of M values (TMM). Differentially expressed genes were identified if a false discovery rate $(F D R)<0.05$ and a two-fold or greater change in transcript levels between control (C) and stress (S) treatment at each time point (C1 vs. S1, C3 vs. S3, and C5 vs. S5) using edgeR Bioconductor (http://www.bioconductor.org)(Robinson et al., 2010). To identify unique and common genes across the three time points, Venn diagrams were generated by VENNY 2.1 (http://bioinfogp.cnb.csic.es/tools/venny/). Gene Ontology enrichment analysis was further performed by Fisher's Exact Test (FDR < 0.05) using Blast2GO (Conesa and Götz, 2008). Kyoto Encyclopedia of Genes and Genomes (KEGG)(Kanehisa and Goto, 2000) was used to characterize associated pathways with threshold bit-score value of 60 (default). Among DEGs, the list of TFs was obtained by using Plant Transcription Factor Database (PlantTFDB, http://planttfdb.cbi.pku.edu.cn)(Yang et al., 2016).

\section{Validation of differentially expressed genes using quantitative PCR}

Five genes with distinct expression changes over three time points were selected to verify the accuracy of RNA-seq data. Expression levels of the selected genes in water-stressed leaves were analyzed by quantitative PCR (qPCR), while the elongation factor $1 \alpha$ (PhEF1 $\alpha$ ) from petunia was used as an internal control (Mallona et al., 2010). Reverse transcription was performed using $\mathrm{qScript}^{\mathrm{TM}}$ cDNA Synthesis Kit (Quanta Biosciences, Gaithersburg, MD, USA) according to the manufacturer's protocol. The qPCR was performed by QuantStudio ${ }^{\mathrm{TM}} 3$ Real-Time PCR System (Thermo Fisher Scientific, Waltham, MA, USA). The PCR reaction mixture contained $10 \mathrm{ng}$ cDNA template, $5 \mu 1$ PowerUp ${ }^{\mathrm{TM}} \mathrm{SYBR}^{\mathrm{TM}}$ Green Mater Mix (Thermo Fisher Scientific, 
Waltham, MA, USA), $0.5 \mu$ f forward and reverse primers $(10 \mu \mathrm{M})$, and water up to $10 \mu 1$. The amplification conditions were as follows: an initial incubation at $50^{\circ} \mathrm{C}$ for $2 \mathrm{~m}$ and $95{ }^{\circ} \mathrm{C}$ for $2 \mathrm{~m}$, followed by 40 cycles of $95{ }^{\circ} \mathrm{C}$ for $15 \mathrm{~s}, 60^{\circ} \mathrm{C}$ for $15 \mathrm{~s}$, and $72{ }^{\circ} \mathrm{C}$ for $1 \mathrm{~m}$. A melting curve (55$95^{\circ} \mathrm{C}$ with a heating rate of $0.15^{\circ} \mathrm{C} \cdot \mathrm{s}^{-1}$ and a continuous fluorescence measurement) was performed for each qPCR reaction. The relative expression of each target gene in one sample

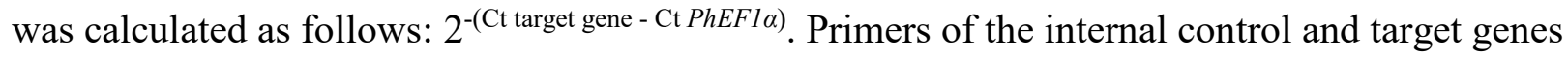
listed in supplementary tables (Table S1), and the PCR product size from each amplicon ranged from 100 to $110 \mathrm{bp}$. All the samples were measured in three technical replications.

\subsection{Results}

\section{Changes in stomatal conductance under water deficit stress}

To determine the duration of water deficit stress treatment, stomatal conductance was measured daily, because stomatal closure is a sign of perception of the stress. Five days after withholding water, stressed plants showed decreased stomatal conductance by $38 \%$ compared to control plants $(P=0.0348$, Fig. 4.1). Leaf samples were collected on day 1,3 , and 5 from wellwatered (control) and water-stressed plants with three replications. Total 18 libraries (two treatments $\times$ three-time points $\times$ three replications per treatment) libraries were used for RNAseq analysis.

Transcriptome sequencing and de novo assembly

Nearly 264 million paired-end reads of 50 bp were generated from 18 libraries, ranging from $8,898,370$ to $18,716,488$ reads per library, with a mean of $14,631,356$ (Table S2). After preprocessing and quality trimming, more than 262 million reads were obtained with the total nucleotides of 13.1 giga bases. De novo assembly using Trinity generated 76,601 contigs with the total contig length of $63,383,628$ bp and average contig length of 827 bp (Table 4.1). In order to remove redundant and/or highly similar contigs, they were then clustered at a sequence similarity threshold value of $90 \%$ using CD-HIT-EST. The final transcriptome library had 72,474 transcripts with the longest length of $16,072 \mathrm{bp}$, an average transcripts size of $803 \mathrm{bp}$, and N50 of 1,377 bp (Table 4.1). Using Bowtie2, 87.6\% of the trimmed reads were mapped back to the transcripts (Table S2), confirming the sequencing data were reliable. 


\section{Functional annotation and classification}

A total of 33,024 (45.6\%) transcripts were annotated against Solanum lycopersicum protein database (Consortium, 2012) using BLASTx. Gene Ontology analysis was performed to classify transcripts based on the possible functions. A total of 25,256 transcripts were classified into 49 different groups belonging to three main categories: cellular component, molecular function, and biological process (Fig. 4.2). Among the three categories, the biological process category was the highest (23), following with the molecular component category (14) and molecular function category (12). In the biological process category, the top two categories were "cellular process" (16.1\%) and "metabolic process" (14.2\%). In molecular function category, "binding" (18.7\%) and "catalytic activity" (16.2\%) were the most abundantly represented. Under the cellular component category, most of the transcripts were involved in "cell" $(19.8 \%)$, "cell part" (19.7\%), and "organelle" (9.7\%).

\section{Differentially expressed genes under water deficit stress}

Gene expression level was analyzed by aligning the trimmed paired-reads to the assembled transcripts. Approximately $88 \%$ of the trimmed reads were mapped to the transcripts (Table S2). Based on the principle component analysis (Fig. S1), one library from each treatment on each day was considered as an outlier, therefore twelve libraries were used for further analyses. Differentially expressed genes were identified by comparing gene expression levels between control and stressed plants at each time point.

The results of hierarchical clustering indicated that differential gene expression was more prominent at the later stage of the treatment $(\mathrm{C} 3, \mathrm{C} 5$, and S3, S5) than at the early stage (C1 and S1)(Fig. 4.3). This result was also supported by the number of genes that were differentially expressed. Significantly higher number of genes were found on day 3 (6417 genes) and 5 (1233 genes) than day 1 (195 genes)(Fig. 4.4A). Under water deficiency, 77 DEGs were commonly upregulated on all three time points, while 69, 2703, and 80 DEGs were upregulated uniquely on day 1, 3, and 5, respectively (Fig. 4.4B). Only 1 DEG was downregulated on all three time points, while 33, 2617, and 71 DEGs were downregulated uniquely on day 1, 3, and 5, respectively (Fig. 4.4B). A total of 6670 DEGs represent approximately 9.2\% $(6670 / 72,474)$ of the total expressed transcripts, and 3742 DEGs were finally annotated. 


\section{Enrichment analysis}

Gene Ontology enrichment analysis were performed to classify biological functions of DEGs under water deficit (Tables 4.2 and 4.3). There were 8, 240, and 15 enriched biological terms from the upregulated process terms in DEGs during water deficit stress (File S1). One biological process term, sulfur compound metabolic process (GO:0006790), was enriched across all three time points, and 6 and 12 biological process terms were enriched in both day 1 and 3, and day 3 and 5, respectively (Table 4.2). The common biological process terms in petunia under water deficit are classified into three categories: metabolic process, biological regulation, and transport (Table 4.2). Metabolic process included sulfate reduction (GO:0019419), sulfate assimilation (GO:0019379), and glutathione catabolic process (GO:0006751). The second category, biological regulation, was associated with homeostatic process (GO:0042592), cell redox homeostasis (GO:0045454), and cellular homeostasis (GO:0019725). The transport category consisted of sulfate transport (GO:0008272) and anion transport (GO:0006820). Enrichment analysis of downregulated genes showed 194 and 88 biological terms on day 3 and 5, while no biological term was enriched on day 1 (Table S2). Sulfur and glutathione metabolic processes (GO:006790 and GO:006749, respectively), and cellular homeostasis (GO:0019725) were enriched among downregulated genes in both day 3 and 5 (Table 4.3). The enriched molecular function and cellular component terms are shown in File S1.

\section{Hormone signal transduction in response to water deficit stress}

Under water deficit, key genes of abscisic acid (ABA) biosynthesis [9-cis-epoxycarotenoid dioxygenase (NCED), xanthoxin dehydrogenase (ABA2), and ABA aldehyde oxidase (AAO)] and catabolism [ABA 8'-hydroxylase (CYP707A)] were differentially expressed on day 3 (Fig. 4.5). Only one of them (ABA2) were upregulated, while others were downregulated three days after withholding water. In addition, the expression of seven genes involved in ABA signal transduction [two pyrabactin-like (PYLs), four phosphatase 2C (PP2Cs), and one ABA response element binding factor (ABF)] were upregulated except one of PYLs (Figs. 4.5 and 4.6). This gene was induced on day 1, but its expression was downregulated on day 3 and 5. It was also identified DEGs involved in ethylene biosynthesis and signal transduction (Figs. 4.5 and 4.6). Three ethylene synthesis-related genes [S-adenosylmethionine synthetase (SAMS) and 1aminocyclopropane-1-carboxylic acid oxidase (ACO)] were induced on days 3 and 5. Also four signal transduction-related genes [ethylene response (ETR) and three ethylene insensitive 
(EIN3)] were upregulated; three of those genes were upregulated on day 3 and one of EIN3 was upregulated on days 3 and 5 (Fig. 4.5).

Transcription factors responding to water deficit stress

In response to dehydration, 63 genes encoding TFs were upregulated and 44 were downregulated. (Fig. 4.7). These TFs were classified into 34 families based on their putative DNA-binding domains. The most abundant TF families were APETALA 2/ethylene response factor (AP2/ERF)(14), no apical meristem, Arabidopsis thaliana activating factor, and cupshaped cotyledon (NAC)(11), myeloblastosis-related (MYB-related)(8), $\mathrm{Cys}_{2}$-His 2 -like fold group (C2H2)(8), basic leucine zipper (bZIP)(7), followed by basic helix-loop-helix (bHLH)(5), homeodomain leucine zipper (HD-ZIP)(5), teosinte branched, cycloidea, and PCF (TCP)(4), and GATA-binding factor (GATA)(4). The most abundant upregulated TF families were AP2/ERF (8), $\mathrm{C} 2 \mathrm{H} 2$ (6), and MYB-related (5), and the most abundant downregulated TF families were NAC (7), AP2/ERF (6), and bHLH (4). Among a total of 63 upregulated TFs, only one TF, growth-regulating factor (GRF) was upregulated on day 1, and other TFs were upregulated on day 3 and/or 5 (Fig. S2). All downregulated TFs were found on day 3 and/or 5, while no TFs were downregulated on day 1 under water deficit stress (Fig. S2). In addition, 101 out of 107 differentially expressed TFs were either induced or suppressed by water stress on day 3 (Fig. S2).

\section{Validation of differentially expressed genes using quantitative PCR}

To confirm RNA-seq data, qPCR was performed with five target genes which showed distinct expression patterns over the three time points. The relative expression levels of target genes had similar expression trend with those from RNA-seq analysis $\left(\mathrm{R}^{2}=0.9389\right.$, Fig. 4.8), which indicated a high reliability of RNA-seq data.

\subsection{Discussion}

Plants have evolved to overcome water deficit stress, such as changes in physiological and biochemical status (Ma et al., 2018). These changes are associated with the differential expression of functional genes (Ma et al., 2018). RNA-seq was performed using leaf tissue of $P$. $\times$ hybrida to analyze transcriptomic changes under water stress. Five days after withholding water, stressed plants reduced stomatal conductance by $38 \%$ compared to control (Fig. 4.1). This physiological change of stomatal closure indicated that plants perceived water stress and initiated 
tolerance mechanisms within five days. Leaves under water deficit stress for 1, 3, and 5 days were used for RNA-seq, and each sampling time point could be defined as immediate, early, and late stages of water deficit, respectively. These time-course transcriptomic data demonstrated the global gene expression profile under water deficit stress in $P . \times$ hybrida.

In total, 262,427,357 high-quality sequencing reads were assembled into 76,601 contigs, and a total of 72,474 transcripts were generated by the program CD-HIT-EST (Table 4.1 and Table S2). The average length of $803 \mathrm{bp}$ and N50 of 1,377 bp are comparable to the results from other de novo transcriptome assemblies (Liu et al., 2016; Ma et al., 2018; Müller et al., 2017; Shi et al., 2011; Villarino et al., 2014; Zhou et al., 2017). BLASTx results were obtained for $45.6 \%$ transcripts against Solanum lycopersicum protein database (Consortium, 2012). This study provided more than 33,000 annotated transcripts, which could be utilized for further research in petunia. Additionally, the qPCR results showed that RNA-seq data was reliable (Fig. 4.8).

Differentially expressed genes were identified under water deficit in petunia by RNA-seq analysis. Of the three time points, the fewest DEGs were found on day 1 (195) and the most DEGs were found on day 3 (6417)(Fig. 4.4A). The hierarchical clustering of samples also showed that the expression profile of stressed plants on day 1 was closer to those of control samples rather than other stressed samples on day 3 and 5 (Fig. 4.3). These results suggested that one day of withholding water might have initiated the water deficit stress response, and gene expression was more active three days after water was withhold. Results of the reduced number of DEGs on day 5 indicated that plants might have acclimated to water deficit. The physiological changes of stomatal closure, the numbers of DEGs on each time point, and the hierarchical clustering pattern coincided with the number of differentially expressed genes on day 1,3 , and 5 as the immediate, early, and late stages of water deficit. The more DEGs were found at least three days after water withholding, showing more genes tended to be consistently upregulated or downregulated to share mechanisms between the early and late stages against water deficit stress. Most of the other transcriptomic analysis under abiotic stress had more upregulated DEGs than downregulated (Bai et al., 2017; Janiak et al., 2018; Li et al., 2015; Liu et al., 2016; Villarino et al., 2014; Zhou et al., 2015).

As water deficit stress occurs, the level of reactive oxygen species (ROS) is increased due to the disturbance between generation and quenching of ROS under stress conditions. Reactive oxygen species causes peroxidation of membrane lipid, degradation of proteins and nucleic acid, 
cellular oxidative damages, and disruption of cellular homeostasis. To mitigate this harmful effect, plants initiate the antioxidant activity to scavenge ROS (Das and Roychoudhury, 2014). Plant cells trigger a complex antioxidant system with a variety of enzymes and non-enzymatic compounds to mitigate the detrimental effects of ROS. The major antioxidative enzymes are superoxide dismutase (SOD), catalase (CAT), peroxidase (POD), ascorbate peroxidase (APX), monodehydroascorbate reductase (MDHAR), dehydroascorbate reductase (DHAR), glutathione reductase (GR), glutathione-S transferase (GST), and glutathione peroxidase (GPX)(Bela et al., 2015; Das and Roychoudhury, 2014). Based on GO enrichment analysis of DEGs, there are homeostasis processes including 'cell redox homeostasis' were dominant terms in both upregulated and downregulated genes under water deficit in petunia (Tables 4.2 and 4.3). In this study, SOD, APX, and MDHAR were downregulated on day 3 (Fig. 4.9), indicating that they might not contribute to scavenge ROS at the early stage of water deficit in petunia. In addition, sulfur/sulfate-related metabolic processes were enriched in DEGs on all three time points (Tables 4.2 and 4.3). Recent studies have highlighted that sulfur metabolism plays crucial roles in cellular homeostasis under water deficit stress (Chan et al., 2013; Couto et al., 2016). Plants uptake sulfur as the form of sulfate, which is converted to adenosine phosphosulfate (APS). At this point, APS can be catalyzed by APS reductase (APR) entering the primary sulfur assimilation pathway or phosphorylated by APS kinase into the secondary assimilation pathways (Chan et al., 2013). In the primary assimilation pathway, glutathione is formed as reduced glutathione (GSH) from cysteine, and GSH functions as a non-enzymatic antioxidant within the cell, resulting in oxidized glutathione (GSSG). Then, GSSG is recycled back to GSH by glutathione reductase (GR)(Chan et al., 2013; Couto et al., 2016). Glutathione reductase is responsible for the recycling of GSSG and maintaining the supply of GSH (Couto et al., 2016). In this study, the expression levels of APR and GR were increased on day 1, 3 and day 3,5, respectively (Fig. 4.9). Additionally, GSH is a substrate for enzymes, GST and GPX, which also attribute to detoxification of ROS (Noctor et al., 2011). Those enzymes were also differentially expressed under water deficit in this study (Fig. 4.9). Twelve GST were identified; half of them were upregulated and the other half were downregulated. Even though they had different expression patterns, one GST was upregulated at all three time points and its expression level was 280 and 139-fold higher than that of control on day 3 and 5, respectively (Fig. 4.9). Downregulated GSTs were identified on day 3 and 5, and the minimum fold change was -6 
compared to control (Fig. 4.9), indicating upregulated GSTs dominated downregulated GSTs. These results suggest that homeostasis processes of sulfur compounds could prevent peroxidation of membrane lipid and damages of cell structures under water deficit response in petunia.

Phytohormones play essential roles in plant acclimation and adaptation to cope with adverse environmental changes (Verma et al., 2016). In this study, the expression levels of genes involved in hormone biosynthesis and signal transduction were changed, including ABA, ethylene, JA, and SA. ABA is known as a major hormone playing crucial roles in abiotic stress responses by inducing stomatal closure and regulating gene expression (Vishwakarma et al., 2017). Previous studies have shown that many genes involved in ABA biosynthesis and signaling were upregulated under water deficit (Dudhate et al., 2018; Liu et al., 2016; Ma et al., 2018; Mashaki et al., 2018; Wang et al., 2018). In this research, among the expression levels of ABA biosynthesis genes (two NCED, ABA2, and AAO), only ABA2 was upregulated (Fig. 4.5). ABA 8' hydroxylase (CYP707A), a catabolic enzyme of ABA, was downregulated under water deficit (Fig. 4.5). Takeuchi et al. (Takeuchi et al., 2016) suggested that inhibition of CYP707A may control ABA mechanisms and increase water deficit tolerance, as shown in Arabidopsis and barley (Millar et al., 2006; Saito et al., 2004). ABA binds to the PYR/PYL/RCAR receptors in order to inhibit the activities of PP2Cs (Fernando and Schroeder, 2016). In this study, a PYL gene was upregulated by approximately 3-fold after one day of water deficit, implying that ABA signal transduction was triggered at immediate stage of water deficit in petunia. In addition, the expression of the key components in the ABA signaling pathway, $\mathrm{PP} 2 \mathrm{C}$ and $\mathrm{ABF}$, were upregulated by 2.3 to 4.4 -fold after 3 and 5 days of water deficit treatment. This result suggested that ABA signal transduction was initiated at immediate stage of water deficit, and ABA regulated stress responses such as stomatal closure on the fifth day after withholding water.

Ethylene is involved in plant growth and development, such as senescence and fruit ripening (Taiz and Zeiger, 2010). In this study, two ethylene biosynthetic enzymes (SAMS and ACO) were upregulated day 3 and 5. Genes involved in ethylene signaling (ETR1 and EIN3) were also upregulated on day 3 and/or 5, activating diverse ethylene responses during the early and late stages of water deficit. Especially, EIN3 induces expression of ethylene responsive factor 1 (ERF1), which is a major TF in plant defense responses in the ethylene signaling pathway (Müller and Munné-Bosch, 2015). Results suggested that ethylene production and signal 
transduction may also be an important water deficit response in petunia. These results were further supported by recent studies. Wan et al. (Wan et al., 2011) reported that ethylene production contributed to water stress tolerance in rice. In other studies ethylene was recognized as another major hormone in plant response to water deficit (Arraes et al., 2015; Hopper et al., 2016; Kazan, 2015; Rabara et al., 2015; Wan et al., 2011).

Several TFs have crucial roles in regulating downstream gene expression, and many TFs have been identified in transcriptome analysis under abiotic stresses (Liu et al., 2016; Ma et al., 2018; Mashaki et al., 2018). The members of AP2/ERF, NAC, MYB-related, C2H2, and bZIP TF families were differentially expressed as found in other studies (Johnson et al., 2014; Liu et al., 2016; Xu et al., 2013b). Especially, AP2/ERF family was mostly differentially expressed in petunia; there were 8 upregulated and 6 downregulated genes in this study (Fig. 4.7). All upregulated AP2/ERF TFs were found on day 3 of water deficit with a fold change range between 3.0 to 233.9 compared to control (Fig. 4.10), indicating that AP2/ERFs play a role in the early response of water deficit. Previous studies have shown that manipulation of AP2/ERF TFs conferred enhanced abiotic stress tolerance. For example, overexpression of an AP2/ERF from Chrysanthemum morifolium, CmERF053, enhanced water deficit stress tolerance in Arabidopsis by showing significantly lower rate of water-loss (Nie et al., 2018), and overexpression of a rice AP2/ERF TF (OsEREBPI) enhanced survival under water deficit and submergence in rice (Jisha et al., 2015). JCERF2 from Jatropha curcas L. enhanced water deficit and salt tolerance in transgenic tobacco with increased free proline and soluble carbohydrate accumulation (Wang et al., 2015). In petunia, AP2/ERF TFs could be good candidates for elucidating water deficit response networks.

\subsection{Conclusion}

With high-throughput and de novo assembly, the comprehensive transcriptome data was obtained from $P$. $\times$ hybrida 'Mitchell Diploid' grown under water deficit stress. Analysis of DEGs provided an excellent database for future genetic and genomic analyses as well as insights into the understanding potential mechanisms of water deficit stress response in petunia. Genes associated with tolerance to water deficit stress were identified and their expression profiles were analyzed over three time points of water deficit. The DEGs included well-known TFs such as AP2/ERF, NAC, MYB-related, and $\mathrm{C} 2 \mathrm{H} 2$, and AP2/ERF family were the most abundant under 
water deficit in petunia. In addition, metabolic processes, sulfur metabolic pathway for antioxidant activity and hormone signal transduction of ABA and ethylene, were enriched in $P$. $\times$ hybrida. Changes in hormone signal transduction may function upstream of water deficit response and then lead to gene expression and physiological changes, such as stomatal closure. These findings will provide a better understanding on molecular mechanisms underlying water deficit tolerance in $P$. $\times$ hybrida, ultimately leading to identify the genes regulating response to water deficit stress. Furthermore, the knowledge obtained from this research can be applied to develop staple crops with enhanced tolerant traits to water deficit stress. 


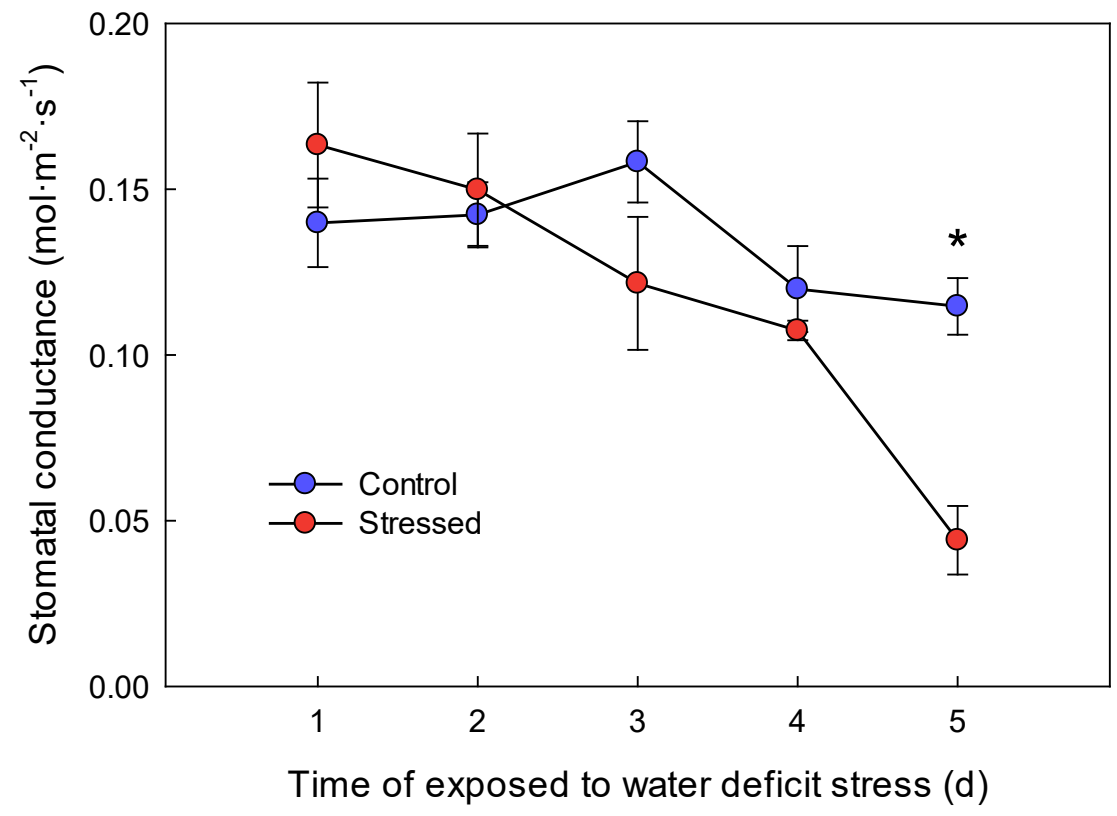

Fig. 4.1. Stomatal conductance of control and water-stressed plants for five days after withholding water. Vertical bars are standard errors of the means with three replications $(n=3)$.

* Significant at $P \leq 0.05$. 
Table 4.1. Summary of assembled contigs by Trinity and clustered transcripts with $90 \%$ similarity.

\begin{tabular}{cll}
\hline Item & Contigs & Transcripts \\
\hline Number of assembled contigs & 76,601 & 72,474 \\
$\leq 500 \mathrm{bp}$ & $40,793(53.3 \%)$ & $39,521(54.5 \%)$ \\
$501-1000 \mathrm{bp}$ & $15,564(20.3 \%)$ & $14,625(20.2 \%)$ \\
$1001-2000 \mathrm{bp}$ & $12,930(16.9 \%)$ & $11,849(16.3 \%)$ \\
$2001-5000 \mathrm{bp}$ & $7,002(9.1 \%)$ & $6,210(8.6 \%)$ \\
$>5001$ bp & $312(0.4 \%)$ & $269(0.4 \%)$ \\
Longest length of contig (bp) & 16,072 & 16,072 \\
Average length of contig (bp) & 827 & 803 \\
N50 (bp) & 1,428 & 1,377 \\
Total length of contigs (bp) & $63,383,628$ & $58,180,257$ \\
\hline
\end{tabular}




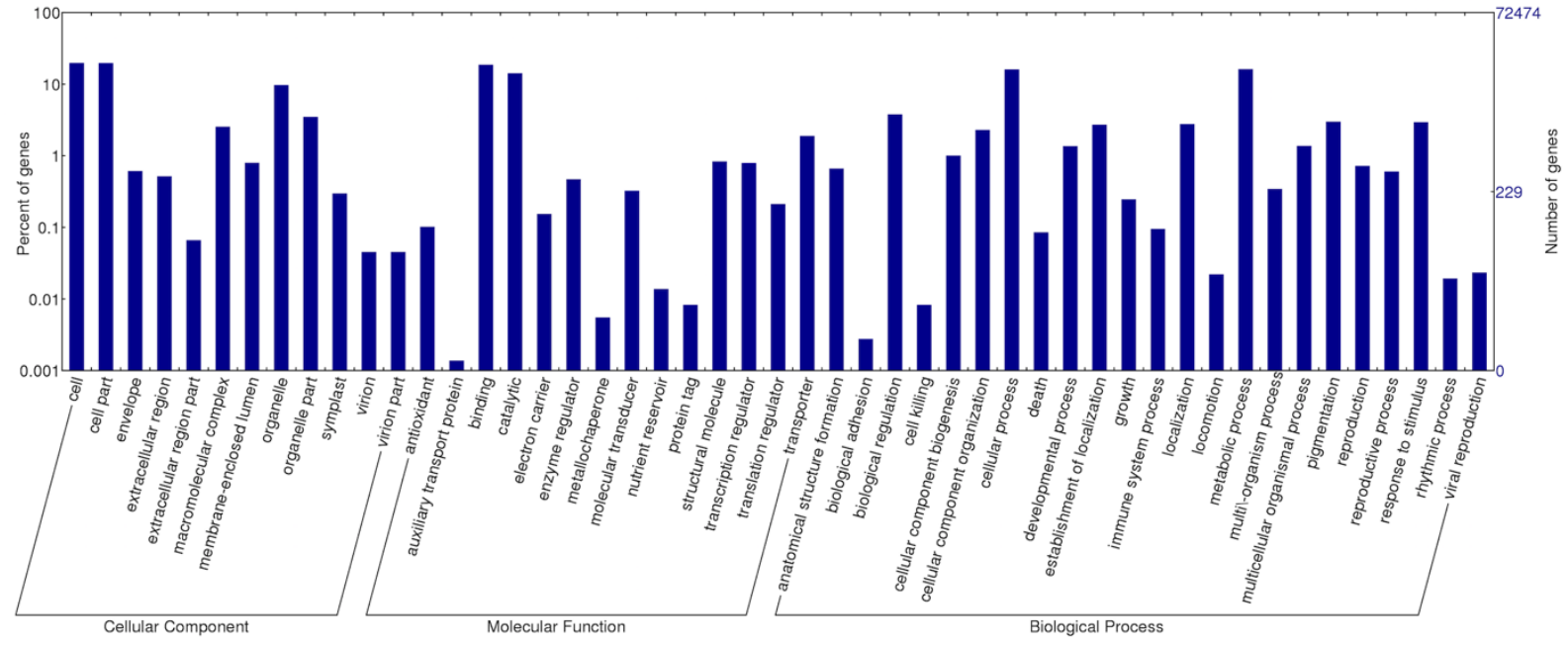

Fig. 4.2. Histogram of Gene Ontology (GO) classification of all transcripts into three main categories including cellular component, molecular function, and biological process. GO classification was performed by Web Gene Ontology Annotation Plot. 


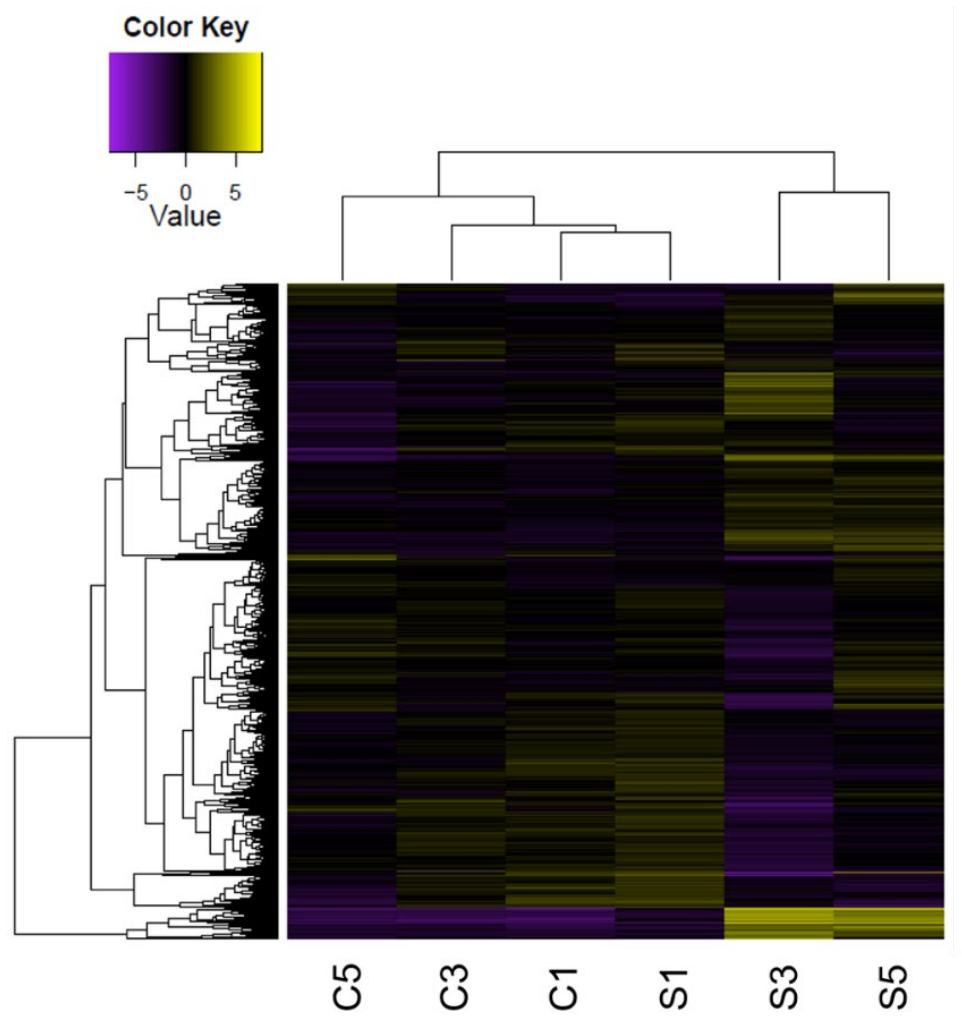

Fig. 4.3. Hierarchical clustering analysis of differentially expressed genes (DEGs). Rows represent individual genes. Genes that increased and decreased in abundance are indicated in yellow and purple, respectively. C and S correspond to control and stressed plants, respectively, and the numbers indicate the number of days of water withholding. DEGs were identified if a two-fold or greater changes in transcript levels between control and stress plants at each time point and a false discovery rate $<0.05$. 


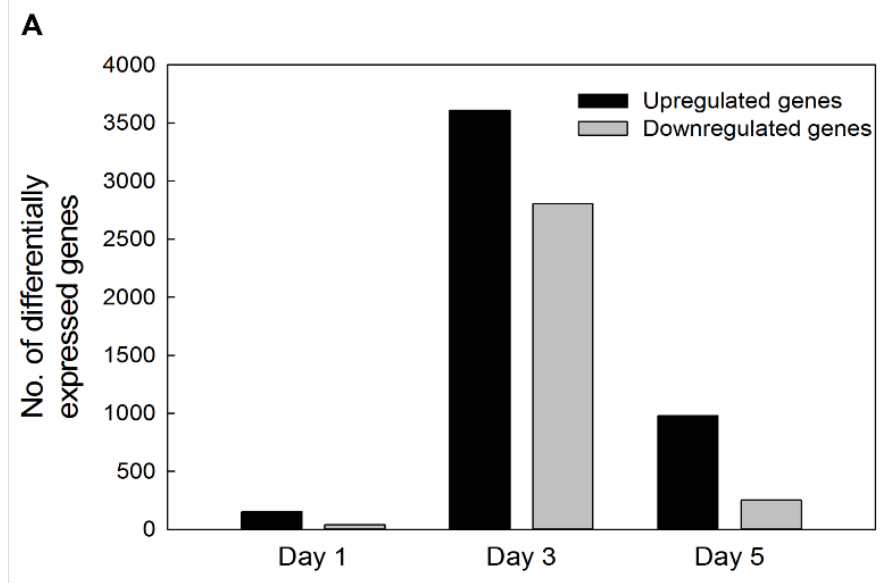

B

Upregulated

Downregulated

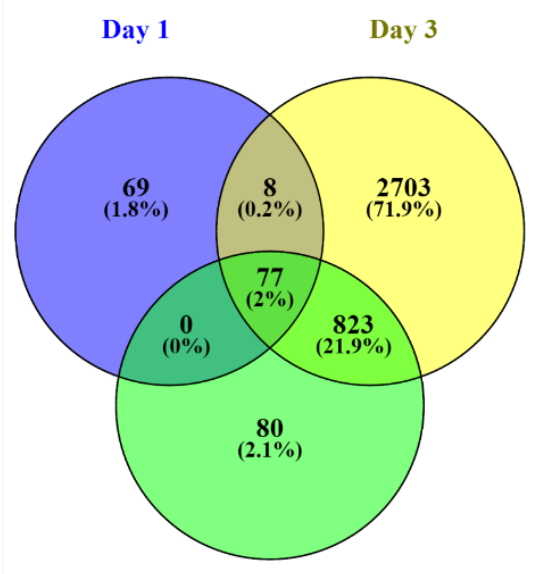

Day 5

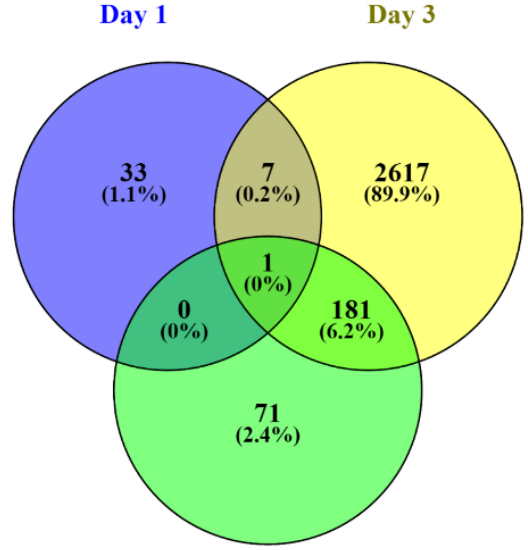

Day 5

Fig. 4.4. Number of differentially expressed genes (DEGs) under water deficit stress. (A)

Number of up or downregulated genes on each day. (B) Venn diagram of upregulated (left) and downregulated (right) genes. DEGs were identified if a two-fold or greater changes in transcript levels between control and stress plants at each time point and a false discovery rate $<0.05$. 
Table 4.2. Biological process terms enriched commonly at least two days by upregulated genes under water deficit stress.

\begin{tabular}{|c|c|c|c|}
\hline Day & Category & GO ID & GO term \\
\hline \multirow[t]{6}{*}{ Day 1 and 3} & Metabolic process & GO:0019419 & sulfate reduction \\
\hline & & GO:0019379 & sulfate assimilation, phosphoadenylyl sulfate reduction by thioredoxin \\
\hline & & GO:0055114 & oxidation-reduction process \\
\hline & Biological regulation & GO:0042592 & homeostatic process \\
\hline & & GO:0045454 & cell redox homeostasis \\
\hline & & GO:0019725 & cellular homeostasis \\
\hline \multirow[t]{12}{*}{ Day 3 and 5} & Metabolic process & GO:0044272 & sulfur compound biosynthetic process \\
\hline & & GO:0006751 & glutathione catabolic process \\
\hline & & GO:0042219 & cellular modified amino acid catabolic process \\
\hline & Transport & GO:0098656 & anion transmembrane transport \\
\hline & & GO:1902358 & sulfate transmembrane transport \\
\hline & & GO:0008272 & sulfate transport \\
\hline & & GO:0006820 & anion transport \\
\hline & & GO:0015698 & inorganic anion transport \\
\hline & & GO:0072348 & sulfur compound transport \\
\hline & & GO:0015103 & inorganic anion transmembrane transport \\
\hline & & GO:0000101 & sulfur amino acid transport \\
\hline & & GO:0015811 & L-cystine transport \\
\hline Day1, 3, and 5 & Metabolic process & GO:0006790 & sulfur compound metabolic process \\
\hline
\end{tabular}


Table 4.3. Biological process terms enriched commonly on day 3 and 5 by downregulated genes under water deficit stress.

\begin{tabular}{llll}
\hline Day & Category & GO ID & GO term \\
\hline Day 3 and 5 & Metabolic process & GO:0005975 & Carbohydrate metabolic process \\
& GO:0006749 & Glutathione metabolic process \\
& GO:0042744 & Hydrogen peroxide catabolic process \\
& GO:0006790 & Sulfur compound metabolic process \\
& GO:0046942 & Carboxylic acid transport \\
\hline & Biological regulation & GO:0019725 & Cellular homeostasis \\
\hline
\end{tabular}




\begin{tabular}{|c|c|c|c|c|c|c|c|}
\hline Hormone & Pathway & Gene & Contig ID & Day1 & Day3 & Day5 & Color key \\
\hline \multirow[t]{13}{*}{ Abscisic acid } & Biosynthesis & NCED & TRINITY_DN15683_c0_g1_i1 & & & & \\
\hline & & & TRINITY_DN1968_c0_g1_i1 & & & & \\
\hline & & ABA2 & TRINITY_DN26350_c0_g1_i2 & & & & $\begin{array}{ll}1 & 1 \\
0 & 4\end{array}$ \\
\hline & & AAO & TRINITY_DN27179_c8_g1_i1 & & & & $\log _{2} \mathrm{FC}$ \\
\hline & Catabolism & CYP707A & TRINITY_DN22070_c1_g1_i1 & & & & \\
\hline & & & TRINITY_DN26120_c0_g2_i1 & & & & \\
\hline & Signal transduction & PYL & TRINITY_DN7122_c0_g1_i1 & & & & \\
\hline & & & TRINITY_DN3202_c0_g1_i1 & & & & \\
\hline & & PP2C & TRINITY_DN14479_c0_g1_i1 & & & & \\
\hline & & & TRINITY_DN15132_c0_g1_i1 & & & & \\
\hline & & & TRINITY_DN20723_c0_g1_i1 & & & & \\
\hline & & & TRINITY_DN25392_c0_g2_i1 & & & & \\
\hline & & $A B F$ & TRINITY_DN26771_c1_g1_i1 & & & & \\
\hline \multirow[t]{7}{*}{ Ethylene } & Biosynthesis & SAMS & TRINITY_DN26765_c3_g2_i1 & & & & \\
\hline & & $\mathrm{ACO}$ & TRINITY_DN23236_c1_g1_i1 & & & & \\
\hline & & & TRINITY_DN23236_c2_g1_i1 & & & & \\
\hline & Signal transduction & ETR1 & TRINITY_DN26448_c0_g1_i1 & & & & \\
\hline & & EIN3 & TRINITY_DN25243_c1_g2_i1 & & & & \\
\hline & & & TRINITY_DN25956_c1_g1_i2 & & & & \\
\hline & & & TRINITY_DN25956_c3_g1_i1 & & & & \\
\hline
\end{tabular}

Fig. 4.5. Heatmap of differentially expressed genes involved in abscisic acid and ethylene biosynthesis and signal transduction pathways under water deficit stress. Expression values of genes are presented as Trimmed Mean of M value (TMM)-normalized $\log _{2}$ fold change (stressed plant/control). Pink and blue colors indicate up and downregulated transcripts under water deficit stress, respectively. 


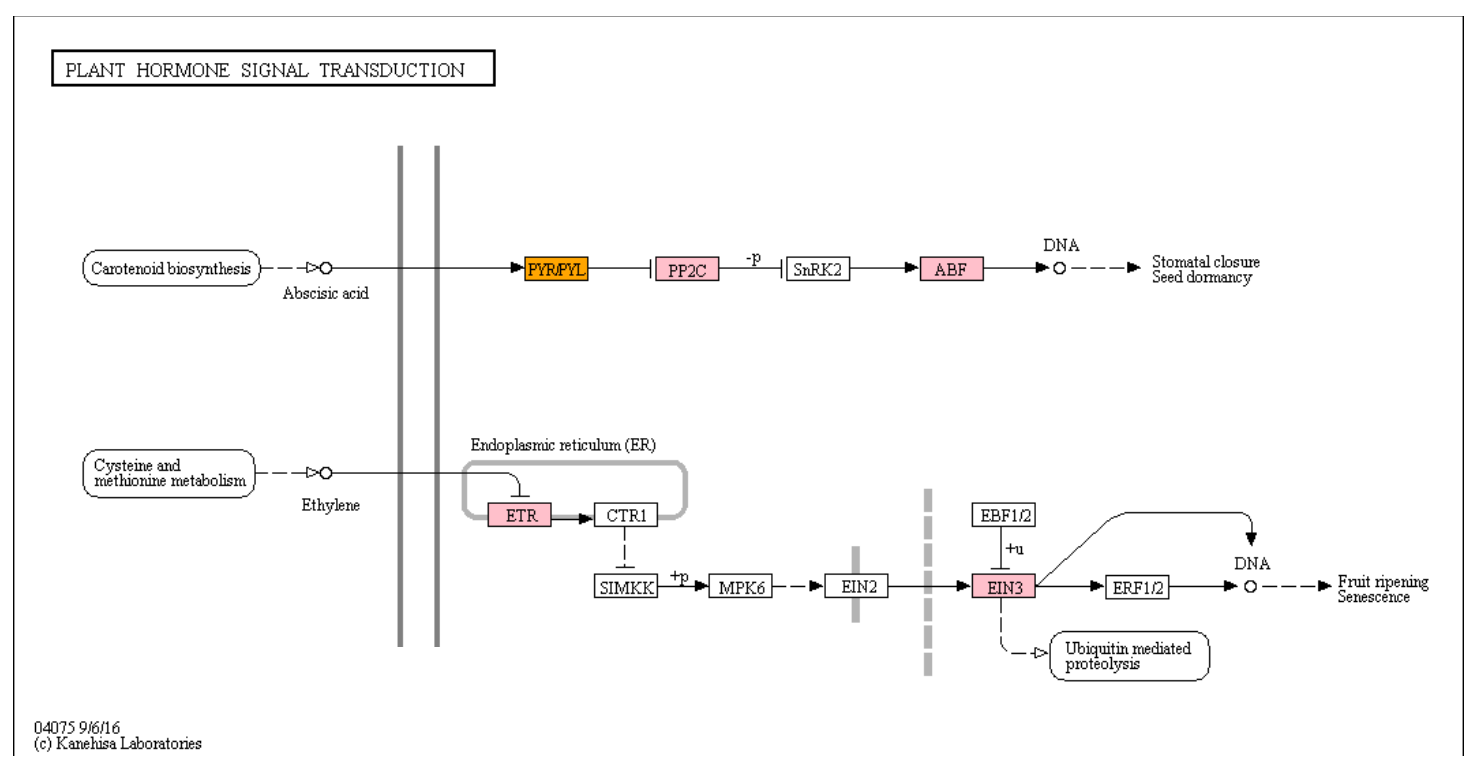

Fig. 4.6. Abscisic acid and ethylene signal transduction Kyoto Encyclopedia of Genes and Genomes pathway. Pink boxes represent genes that were upregulated under water deficit stress, while the orange box indicate genes which were up and downregulated during five days of water deficit. 


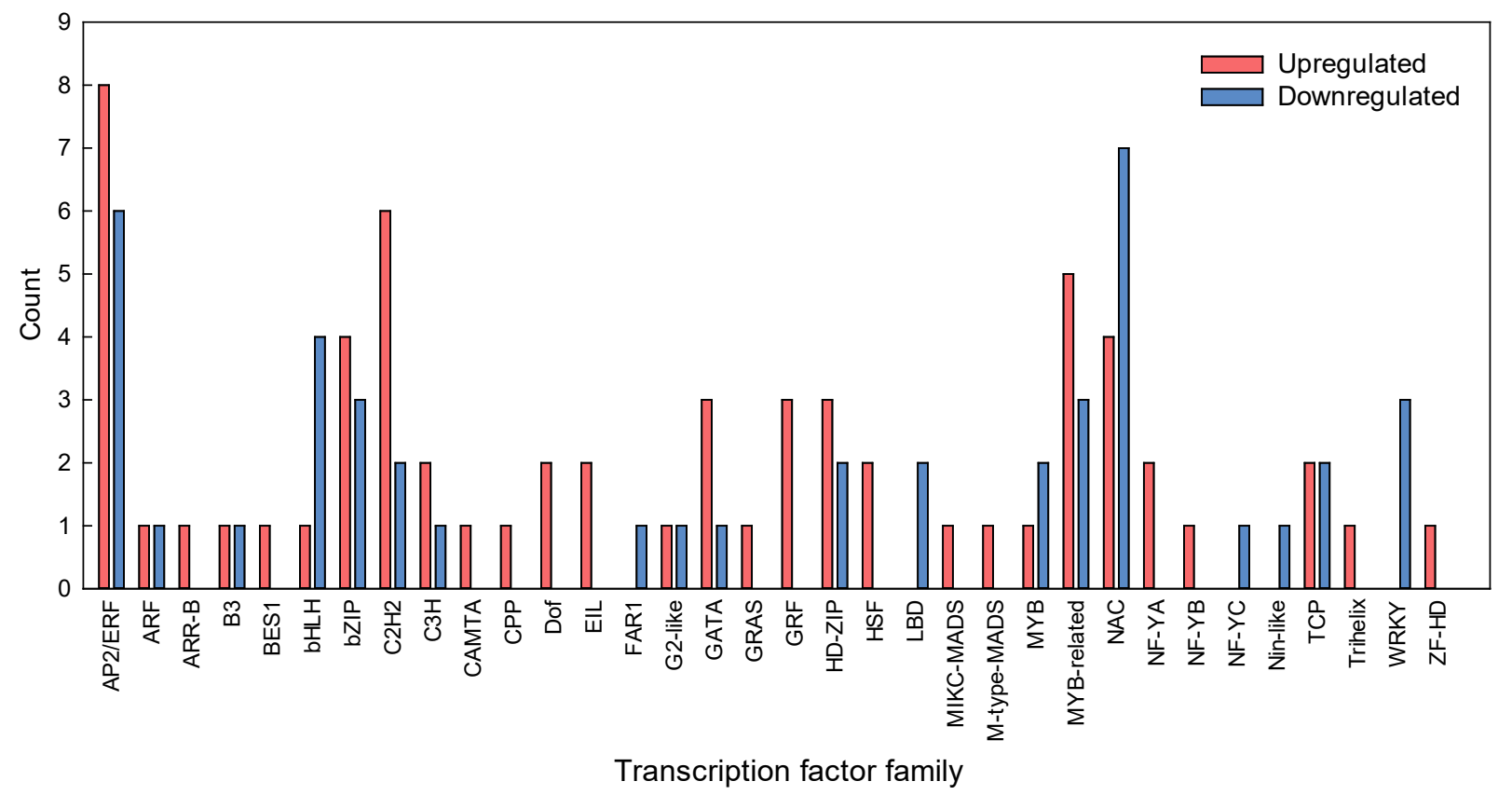

Fig. 4.7. Distribution of differentially expressed transcription factor (TF) families. Pink bars indicate upregulated TFs and blue bars indicate downregulated TFs under water deficit stress. 


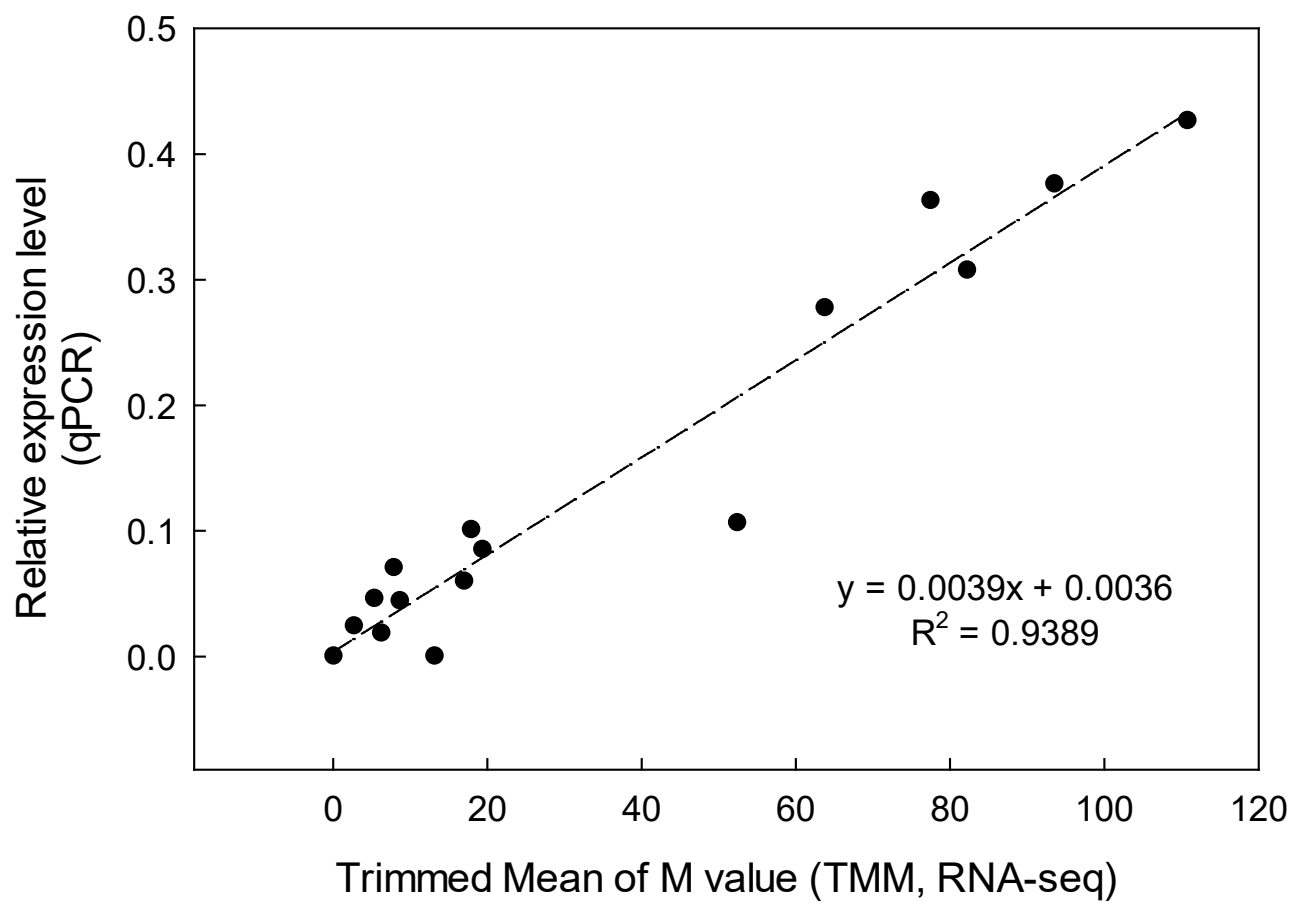

Fig. 4.8. Consistency between RNA-seq data and qPCR data. 


\begin{tabular}{|c|c|c|c|c|c|}
\hline Gene & Contig ID & Day1 & Day2 & Day3 & Color key \\
\hline \multirow[t]{2}{*}{ SOD } & TRINITY_DN14341_c0_g1_i1 & & & & \\
\hline & TRINITY_DN32325_c0_g1_i1 & & & & \\
\hline APX & TRINITY_DN25729_c0_g1_i1 & & & & $\begin{array}{ccc}1 & 1 & 1 \\
-2 & 0 & 2\end{array}$ \\
\hline MDHAR & TRINITY_DN33361_c0_g1_i1 & & & & $\log _{2} \mathrm{FC}$ \\
\hline APR & TRINITY_DN26094_c0_g1_i1 & & & & \\
\hline GR & TRINITY_DN26463_c1_g1_i1 & & & & \\
\hline \multirow[t]{12}{*}{ GST } & TRINITY_DN19429_c1_g1_i1 & & & & \\
\hline & TRINITY_DN3888_c0_g1_i1 & & & & \\
\hline & TRINITY_DN9876_c0_g1_i1 & & & & \\
\hline & TRINITY_DN22615_c0_g1_i1 & & & & \\
\hline & TRINITY_DN25690_c2_g1_i1 & & & & \\
\hline & TRINITY_DN8250_c0_g1_i1 & & & & \\
\hline & TRINITY_DN27244_c3_g1_i1 & & & & \\
\hline & TRINITY_DN27062_c0_g1_i1 & & & & \\
\hline & TRINITY_DN25112_c0_g1_i1 & & & & \\
\hline & TRINITY_DN5495_c0_g1_i1 & & & & \\
\hline & TRINITY_DN18044_c0_g1_i1 & & & & \\
\hline & TRINITY_DN23908_c0_g1_i1 & & & & \\
\hline \multirow[t]{2}{*}{ GPX } & TRINITY_DN23635_c0_g2_i1 & & & & \\
\hline & TRINITY_DN30432_c0_g1_i1 & & & & \\
\hline
\end{tabular}

Fig. 4.9. Heatmap of differentially expressed genes involved in redox homeostasis processes under water deficit stress. Expression values of genes are presented as Trimmed Mean of M value (TMM)-normalized $\log _{2}$ fold change (stressed plantcontrol). Pink and blue colors indicate up and downregulated transcripts under water deficit stress, respectively. 


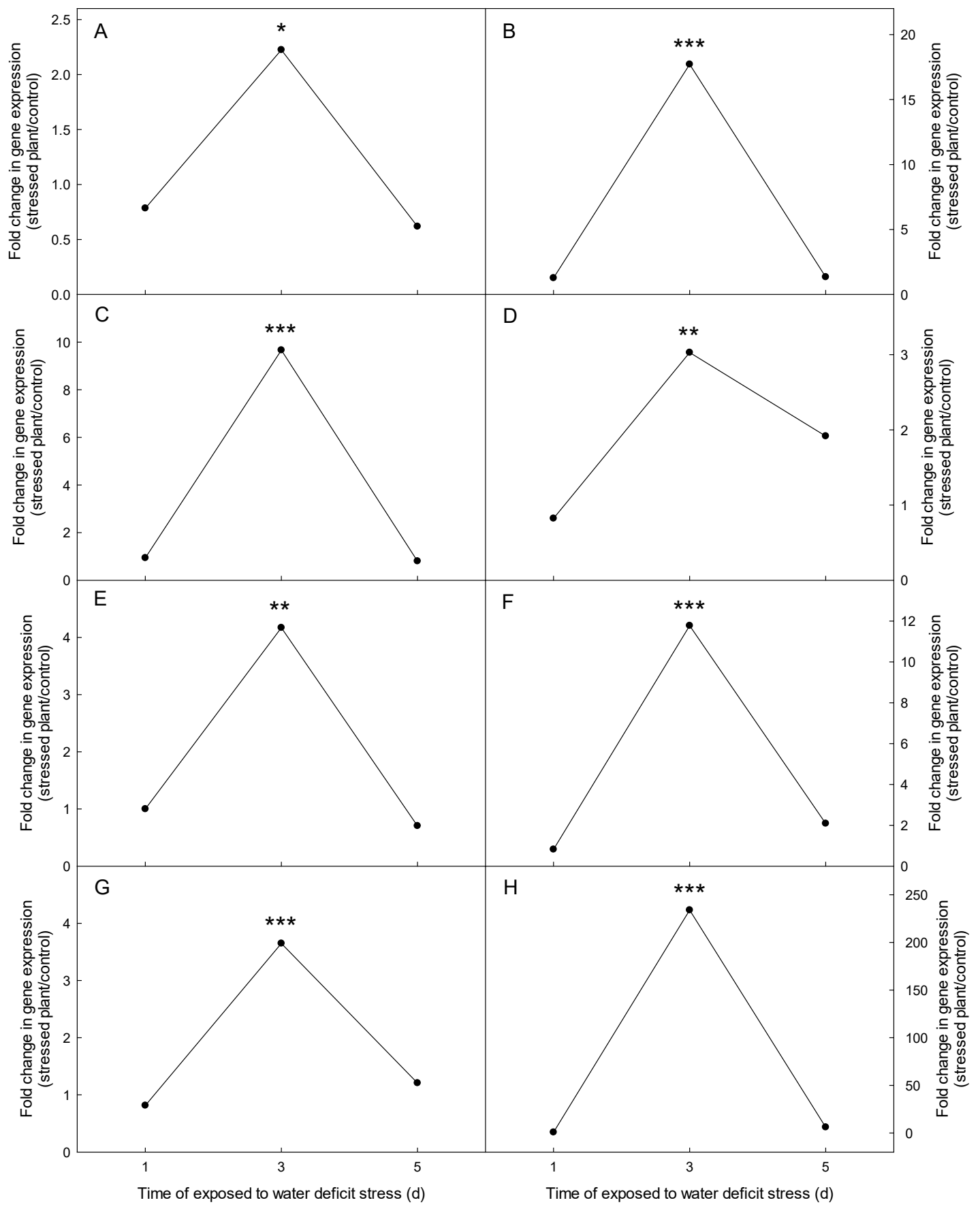

Fig. 4.10. Fold change in gene expression of AP2/ERF TFs under water deficit stress compared to control. Contig IDs are TRINITY_DN11899_c0_g2_i1 (A), TRINITY_DN14217_c0_g1_i1 (B), TRINITY_DN22124_c0_g1_i1 (C), TRINITY_DN24527_c0_g1_i1 (D), TRINITY_DN25125_c2_g1_i1 (E), TRINITY_DN30613_c0_g1_i1 (F), TRINITY_DN32633_c0_g1_i1 (G), and TRINITY_DN33611_c0_g1_i1 (H). *, **, *** Significant at false discovery rate $<0.05,0.01$, and 0.001 , respectively. 


\section{Chapter 5 Functional analysis of transcription factor PhERF039 for enhancing water stress tolerance in Petunia $\times$ hybrida 'Mitchell Diploid'}

\subsection{Abstract}

Ethylene-responsive factors (ERFs) are important plant transcription factors (TFs) that regulate plant responses against various abiotic stresses. However, little information of $E R F$ genes involved in abiotic stress is available in petunia (Petunia $\times$ hybrida). In this study, a petunia $E R F$ gene, $P h E R F 039$, was cloned and functional analysis was performed. The quantitative PCR analysis revealed that PhERF039 was induced at the early stage of water deficit stress. Under-expression of PhERF039 (UE) exhibited rosette growth habit, higher number of branches, and delayed flowering compared to the wild type (WT). The UE petunia was evaluated under various volumetric water contents $(\theta): 0.25,0.15,0.10$, or $0.05 \mathrm{~m}^{3} \cdot \mathrm{m}^{-3}$ using an automated irrigation system. Transgenic plants did not delay plant wilting, but the $\theta$ for UE reached to the set point later than that for WT. A lower stomatal conductance was observed in UE than WT under all treatments. These results suggested that PhERF039 could be involved in plant responses under water deficit by regulating stomatal movements as well as branching pattern and flower development.

\subsection{Introduction}

Petunia (Petunia $\times$ hybrida) is one of the most popular ornamental crops worldwide in landscaping. In the United States, it is the top bedding plant and the value of petunia at wholesale is estimated at $\$ 142$ million for 2018 (USDA, 2019). Even though petunias are grown under optimum environmental conditions in greenhouse, their quality could be diminished if they were not taken care of during the postproduction period. Water deficit is a major environmental factor that negatively affects plant growth and production. It is important to understand the responses of petunia to water deficit stress and to breed petunia cultivars with enhanced water deficit tolerance.

Plants are sessile and survive from harsh environmental conditions. To survive under the adverse environments, plants have evolved sophisticated networks to perceive stress and regulate the expression of stress-responsive genes (Farooq et al., 2009). Transcription factors (TFs) act as key controllers by regulating expression of downstream genes and consequently enhance stress tolerance in plants (Hadiarto and Tran, 2011). In recent years, a wide range of TF families 
relevant to water deficit stress response have been identified. Among the TFs, the APETALA $\underline{2}$ /ethylene-responsive factor (AP2/ERF) family is a large gene family of plant-specific TFs. The AP2/ERF family is involved in response to water deficit, salt, high and cold temperature stresses and disease resistance (Mizoi et al., 2012).

The AP2/ERF family can be classified into four major subfamilies: AP2, ERF, related to abscisic acid and viviparous $1(\mathrm{RAV})$, and dehydration-responsive element-binding proteins (DREBs)(Xie et al., 2019). Of these, many stress-inducible ERF subfamily members have been isolated and characterized. Recent research suggests that ERF members play diverse roles in plant adaptation mechanisms to both abiotic and biotic stresses (Li et al., 2018b; Nie et al., 2018;

Quan et al., 2010; Rong et al., 2014). For example, overexpression of wheat TaERF3 exhibited enhanced tolerance to salt and water deficit stresses by increasing proline accumulation and inducing stomatal closure, while TaERF3-silencing plants displayed more sensitivity to both stresses (Rong et al., 2014). A tomato ERF protein TSRF1 improved the osmotic and drought tolerance in rice seedling by increasing the amount of proline and soluble sugars, and also induced the expression of pathogenesis-related genes (Quan et al., 2010). In contrast, a cotton GhERF38 reduced seed germination rate, chlorophyll content, and survival rate under salt and water deficit stresses (Ma et al., 2017).

To understand mechanisms of water deficit tolerance, RNAseq was performed using petunia grown under water deficit condition. The transcriptome revealed 6,679 unigenes under water deficit, and 107 TFs were identified to be associated with response to water deficit. Fifty-five TFs were upregulated at the early stage of water deficit stress, and eight AP2/ERF TFs were identified in petunia. Among them, PhERF039, a member of AP2/ERF family, was selected for functional analysis. In this study, the roles of PhERF039 gene involved in water deficit stress were further investigated.

\subsection{Materials and methods}

\section{Expression of PhERF039 under water deficit stress}

P. $\times$ hybrida 'Mitchell Diploid' (MD) seedling were grown in 11-cm pots with soilless media (Sunshine ${ }^{\circledR}$ Mix \#1; Sun Gro Horticulture, Agawam, MA, USA) at a greenhouse (Morgantown, WV, USA). Nine-week-old petunias were subjected to water deficit stress by withholding irrigation with three biological replications. Transcriptomic analysis was performed with leaf 
samples collected 1, 3, and 5 days after withholding water, and it was shown that PhERF039 were upregulated under water deficit. The actual expression level of PhERF039 were analyzed. Total RNA was isolated with Trizol (Invitrogen, USA) and treated with DNase I (Thermo Fisher Scientific, Waltham, MA, USA). First cDNA was prepared from $1 \mu \mathrm{g}$ of total RNA with qScript ${ }^{\mathrm{TM}}$ cDNA Synthesis Kit (Quanta Biosciences, Gaithersburg, MD, USA). The transcript levels of PhERF039 were quantified using QuantStudio ${ }^{\text {TM }} 3$ Real-Time PCR System (Thermo Fisher Scientific, Waltham, MA, USA) with PowerUp ${ }^{\mathrm{TM}} \mathrm{SYBR}^{\mathrm{TM}}$ Green Mater Mix (Thermo Fisher Scientific, Waltham, MA, USA). The amplification conditions were as follows: an initial incubation at $50^{\circ} \mathrm{C}$ for $2 \mathrm{~m}$ and $95^{\circ} \mathrm{C}$ for $2 \mathrm{~m}$, followed by 40 cycles of $95{ }^{\circ} \mathrm{C}$ for $15 \mathrm{~s}, 60^{\circ} \mathrm{C}$ for $15 \mathrm{~s}$, and $72{ }^{\circ} \mathrm{C}$ for $1 \mathrm{~min}$. Primers used to determine PhERF039 expression were 5'TGACCGTTATCAATACCCAACTT-3' as a forward primer (F) and 5'CCACTAATAGCACTCCCACAAA-3' as a reverse primer (R). PhEFl $\alpha$ was used as an internal control and the primers were 5'-CCTGGTCAAATTGGAAACGG-3' (forward primer) and 5'CAGATCGCCTGTCAATCTTGG-3' (reverse primer). A melting curve $\left(55-95^{\circ} \mathrm{C}\right.$ with a heating rate of $0.15{ }^{\circ} \mathrm{C} \cdot \mathrm{s}^{-1}$ and a continuous fluorescence measurement) was performed for each quantitative real-time PCR (qRT-PCR) reaction. The $2^{-\Delta \mathrm{Ct}}$ method was used for the analysis. All the samples were measured in three technical replications. The transcript levels of PhERF039 in non-transgenic MD and transgenic line were quantified as described above.

\section{Cloning, construction of plasmid and plant transformation}

Using the total RNA extracted from the leaf tissue, the first strand cDNA containing the open reading frame (ORF) of PhERF039 was generated using a pair of primers: 5'TGTGGTCTCAAATGGAAGATCATCATCTTCATCATG-3' and 5'TGTGGTCTCAAAGCTAATAGCACTCCCACAAAAATG-3' (the underlined bases are for a restriction site of $B s a I)$. The deduced amino acids sequences of PhERF039 were aligned with homologs from other plant species. The PCR fragment was digested by BsaI and ligated into the binary expression vector pICH86988 (Addgene plasmid 48076). The expression of the gene was driven by the CaMV35S promoter. This construct was transformed into $P$. $\times$ hybrida 'Mitchell Diploid' leaf tissue using Agrobacterium (A.tumefaciens LBA4404)-mediated transformation according to the established protocol with modification (Conner et al., 2009). Positive transgenic plants were verified by using a primer set that spanned the PhERF039 insert to identify the 
transgene. The verification primer set was 5'-GAGGACACGCTCGAGTATAA-3' and 5'GTAAGGATCTGAGCTACACATG-3'.

\section{Measurements of growth and development}

Evaluation of plant growth and development was performed on wild type (WT) and $\mathrm{T}_{1}$ generation of the PhERF039 transgenic line. Growth index (GI) was calculated by the following equation: GI $=[\{($ canopy widest width + perpendicular width $) / 2\}+$ height $] / 2$. Relative chlorophyll content of fully expanded leaves (seventh or eighth leaf from the top) was measured using a SPAD-502 Chlorophyll Meter (Konica Minolta Sensing, Inc., Osaka, Japan). The flowering time was determined as the number of days from the sowing date until the first flower was fully opened. The number of flowers per plants was counted. The flower size was determined as the average of the widest width and perpendicular width of a flower. The flower longevity was determined as the time from anthesis until the corolla was completed wilted (Chang et al., 2014). Five flowers from different plants were used for flower size and longevity measurements. Plants were blocked in two growth chambers and each chamber had two plants per treatment. The average temperatures of one chamber were $20.7 / 18.6 \pm 0.2 / 1.3{ }^{\circ} \mathrm{C}$ day/night (mean $\pm \mathrm{SD}$ ) with relative humidity of $65.8 \% \pm 5.9 \%$, photosynthetic photon flux density (PPFD) of $204 \mu \mathrm{mol} \cdot \mathrm{m}^{-2} \cdot \mathrm{s}^{-1}$ from 0600 to $2000 \mathrm{HR}$ daily, and $\mathrm{CO}_{2}$ at $470 \pm 52 \mu \mathrm{mol} \cdot \mathrm{m}^{-2} \cdot \mathrm{s}^{-1}$ (mean $\pm \mathrm{SD}$ ). For the other chamber, the average temperatures were $20.5 / 18.6 \pm 1.0 / 0.7^{\circ} \mathrm{C}$ day $/$ night (mean $\pm \mathrm{SD}$ ) with relative humidity of $67.6 \% \pm 4.1 \%, P P F D$ of $208 \mu \mathrm{mol} \cdot \mathrm{m}^{-2} \cdot \mathrm{s}^{-1}$ from 0600 to $2000 \mathrm{HR}$ daily, and $\mathrm{CO}_{2}$ at $472 \pm 60 \mu \mathrm{mol} \cdot \mathrm{m}^{-2} \cdot \mathrm{s}^{-1}$ (mean $\pm \mathrm{SD}$ ). Data are the means of measurements from two replications $(\mathrm{n}=2)$.

Water deficit stress treatment and measurements under water stress

Wild type and the transgenic line of petunia were evaluated under the various levels of volumetric water content $(\theta): 0.25,0.15,0.10$, or $0.05 \mathrm{~m}^{3} \cdot \mathrm{m}^{-3}$ in growing media. The moisture levels of the growing media were maintained by automated irrigation system (Nemali and van Iersel, 2006). The visual wilt status was measured daily based on the ratings described by Waterland et al. (2010a). Wilt status ratings were from 1 to 5 with $5=$ completely turgid, $4=$ soft to the touch but still upright, $3=$ starting to wilt, $2=$ severely wilted, and $1=$ wilted to the point that leaves are dried and desiccated (Waterland et al., 2010a). Stomatal conductance ( $g \mathrm{~s}$ ) was measured daily with a portable photosynthesis system (LI-6400XT; LI-COR, Lincoln, NE). A leaf was clamped into an extended chamber with clear top and bottom covers (Extended Reach 1 
cm Chamber LI6400-15; LI-COR). Environmental conditions in the chamber were set at 400 $\mu \mathrm{mol} \cdot \mathrm{m}^{-2} \cdot \mathrm{s}^{-1} \mathrm{CO}_{2}$, and $25^{\circ} \mathrm{C}$ as the block temperature. Readings were conducted from 1000 to $1400 \mathrm{HR}$.

\section{Statistical analysis}

The experiment design was a randomized complete block design were conducted. Plants were blocked in two growth chambers $(\mathrm{n}=2)$ and each chamber had two plants per treatment. Statistical analyses were performed using SAS (version 9.3; SAS Institute, Cary, NC). Differences between WT and transgenic line were assessed by Tukey's test at $P \leq 0.05$.

\subsection{Results and Discussion}

\section{Isolation and sequence analysis of PhERF039}

To identify the genes regulating water deficit stress response, RNA sequencing was performed with petunia grown under water deficit condition. The transcriptome revealed 6,679 unigenes differentially expressed under water deficit, and $107 \mathrm{TFs}$ in $34 \mathrm{TF}$ families were differentially expressed under water deficit. Among them, the AP2/ERF family was the most abundant, and eight upregulated AP2/ERF TFs were identified under water deficit in petunia. One member of this family, PhERF039, was selected for functional analysis. The length of ORF of PhERF039 is $816 \mathrm{bp}$, and it encodes a predicted protein of 271 amino acids ( $30.2 \mathrm{KDa}$ ). The amino acid sequence alignment analysis showed that PhERF039 contains a highly conserved AP2 domain and a high degree of sequence homology to NtERF039-like in Nicotiana tabacum (tobacco, 71\% identity), SlERF038-like in Solanum lycopersicum (tomato, 67\% identity), CaERF034-like in Capsicum annuum (pepper, 62\% identity), AtERF38 in Arabidopsis thaliana (52\% identity), and GhERF38 in Gossypium hirsutum (cotton, 52\% identity)(Fig. 5.1). Among them, GhERF38 were characterized under abiotic stresses (Ma et al., 2017).

\section{Expression of PhERF039 under water deficit stress.}

The temporal expression pattern of PhERF039 was analyzed by qRT-PCR. Petunias were exposed to stress by withholding water for 5 days, and the stressed plants reduced $g s 5$ days after water deficit (Chapter 4, Fig. 4.1). The transcript level of PhERF039 was markedly elevated 3 days after water deficit compared to that of the control (Fig. 5.2). There was no difference in PhERF039 transcript levels between the control and the stressed plants 1 and 5 days after water deficit. The results of $g s$ indicated that plants perceived water deficit and induced stomatal 
closure to limit water loss and tolerate the stress. The increased transcript level of PhERF039 suggested the possible role of PhERF039 in the responses of water deficit stress. Övernäs (2010) reported that $A t E R F 38$ was induced under salt and cold stresses but not by abscisic acid (ABA) treatment, suggesting AtERF38 is involved in stress responses through an ABA-independent pathway. GhERF38 was upregulated under abiotic stresses (salt and water deficit) and additionally by ABA treatment (Ma et al., 2017).

Expression level of PhERF039 in transgenic plants

To analyze the function of PhERF039, Agrobacterium-mediated transformation was used to overexpress PhERF039 in petunia. The gene expression was driven by CaMV 35S promoter. One transgenic line $\left(\mathrm{T}_{0}\right)$ was obtained, and the integration of PhERF039 was confirmed by PCR (Fig. 5.3). The $T_{1}$ seeds of the transgenic line were sown, and $17 \mathrm{~T}_{1}$ plants were verified to have the integration of PhERF039 out of $24 \mathrm{~T}_{1}$ plants (70.8\%). Sixteen $\mathrm{T}_{1}$ plants with the PhERF039 integration were used for this experiment (Fig. 5.3). The introduction of PhERF039 was expected to increase its expression level; however, the expression of PhERF039 was reduced in $\mathrm{T}_{1}$ generations compared to wild type (WT)(Fig. 5.4). It is suspected that the expression of transgene (PhERF039) degraded mRNA of both endogenous and the transgene. This phenomenon has been well documented as RNA interference (RNAi)(Hannon, 2002). It was reported in petunia that white flowers and/or mixture of white and purple flowers were produced by the introduction of a chimeric chalcone synthase expected to produce darker purple flowers (Napoli et al., 1990). Later, the molecular mechanism was identified that the double-stranded RNAs (dsRNAs) from transgene led the destruction of mRNAs containing the similar sequences, and such dsRNA-mediated silencing are being employed for functional analysis of target genes (Wilson and Doudna, 2013). Instead of overexpressing, under-expression line of PhERF039 transgenic plants (UE) were obtained for functional analysis.

\section{Effects of under-expression of PhERF039 on plant growth and development}

The UE produced more rosette branches compared to the WT (Fig. 5.5A and B). After 21 weeks of growth, WT plants displayed approximately six branches, while the UE produced approximately nine branches $(P=0.0002)$ (Fig. 5.5B). Growth index (GI) of transgenic plants was smaller than that of WT on week $15(P<0.0001)$ and $21(P<0.0001)$ (Fig. 5.5C). The leaf chlorophyll concentrations of the transgenic plants significantly increased compared to WT on week 15 and $21(P<0.0001$ and $P=0.0048$, respectively)(Fig. 5.5D). The UE plants exhibited 
delayed flowering by 20 days compared to WT ( $P<0.0001)$ (Fig. 5.6A). The number of flowers were significantly higher in UE than WT on week $24(P<0.0433)$, but the difference became insignificant on week 28 (Fig. 5.6B). The flower size and longevity were not different between WT and UE (Fig. 5.6C and D).

Wild type plants displayed strong apical dominance showing high canopy height and larger GI, while UE plants produced a number of lateral branches. The shoot morphology of UE suggested that PhERF039 could inhibit the development of shoot branching. It has been known that plant hormones, auxin and cytokinins (CKs), regulate bud outgrowth. Auxin induces apical dominance and inhibits shoot branching, while CKs antagonize auxin in apical dominance by triggering outgrowth (Müller and Leyser, 2011). Some studies have revealed that the $A P 2 / E R F$ is involved in plant shoot morphological characteristics, possibly through the dynamic regulation of CKs homeostasis and responses (Gupta and Rashotte, 2014; Raines et al., 2016). Haver et al. (2002) reported that the exposure of ethylene inhibited apical dominance and promoted the outgrowth of lateral shoots by decreasing the auxin/cytokinin ratio in $P$. $\times$ hybrida 'Orchid'. These results indicated that PhERF039 might play an opposite role to ethylene effects on regulating shoot branching through auxin and CKs signaling. The relative chlorophyll content was increased in UE, suggesting that PhERF039 might be involved in triggering leaf senescence through chlorophyll degradation and its impaired synthesis (Iqbal et al., 2017). The UE also delayed flowering compared to WT. Flowering is regulated by complex gene regulation networks that integrate multiple environmental conditions and endogenous signals as well as hormonal signaling (Campos-Rivero et al., 2017). The effects of ethylene in the regulation of flower development are complicated to investigate. The mutation of CTRl, a key negative regulator in ethylene signaling, delayed flowering, suggesting that ethylene inhibited flowering in Arabidopsis (Achard et al., 2007). However, ethylene induced a reproductive transition in rice (Wuriyanghan et al., 2009). The number of flowers are also affected by ethylene, but the sensitivity to ethylene on flower number is not clear as well as flowering time (Iqbal et al., 2017). To identify the functional role of PhERF039 in growth and flowering time, plant hormone networks and their cross-talk should be taken into consideration in future research.

\section{Effects of the under-expression line of PhERF039 on water deficit tolerance}

Twenty-one-week-old plants were subjected to water treatments for 7 days with various $\theta \mathrm{s}$ : $0.25,0.15,0.10$, or $0.05 \mathrm{~m}^{3} \cdot \mathrm{m}^{-3}$. The WT plants seemed to reach the set $\theta$ points earlier than UE 
plants, approximately 1 to 2 days earlier (Fig. 5.7). Both WT and UE at 0.25 and $0.15 \mathrm{~m}^{3} \cdot \mathrm{m}^{-3}$ maintained a visual wilt status rating of 5 (Fig. 5.8A and B). The wilt status values were not significantly different between WT and UE at all $\theta$ s (Fig. 5.8). However, UE plants had lower gs than WT plants up to three days after treatment at all treatment conditions (Fig. 5.9). When plants were growing in the drier media $\left(\theta \mathrm{s}\right.$ at 0.01 and $\left.0.05 \mathrm{~m}^{3} \cdot \mathrm{m}^{-3}\right)$, UE plants maintained lower stomatal conductance up to five days after treatment (Fig. 5.9C and D). Overall, the underexpression line of PhERF039 exhibited lower stomatal conductance than WT, indicating that PhERF039 might be negatively involved in stomatal closure.

Regression analyses were performed using the data obtained from wilt status in Fig. 5.8D and gs in Fig. 5.9D measured at the lowest volumetric water content treatment $\left(0.05 \mathrm{~m}^{3} \cdot \mathrm{m}^{-3}\right)$ in Fig. 5.7. Plant wilt status was positively correlated with $\theta$, and there is no distinct difference in slopes and intercepts of the two regression models between WT and UE $(P=0.7554$ and 0.5258 , respectively)(Fig. 5.10). When subject to water deficit stress, both WT and UE exhibited a decrease in gs as volumetric water content in the media gradually decreased to the set point at $0.05 \mathrm{~m}^{3} \cdot \mathrm{m}^{-3}$ (Fig. 5.11). The slope of WT was higher than that of UE $(P<0.0001)$ (Fig. 5.11).

According the regression models of $\theta$ in substrate and wilt status, UE did not increase the wilt status compared to WT (Fig. 5.10), while stomatal conductance was decreased in UE (Fig. 5.11). These results indicated that UE plants did not delay plant wilting compared to WT plants. However, the volumetric water content of the media for UE reached the set threshold later than that for WT. In general, UE plants maintained lower gs than WT (Fig. 5.11). Plants lose about $97 \%$ of total water absorbed from roots through transpiration. The transpirational rate is determined largely by gs (Li et al., 2018b). Many researches have addressed that inducing stomatal closure or blocking stomata enhanced water deficit tolerance by limiting transpirational water loss (Park et al., 2016; Waterland et al., 2010a; Waterland et al., 2010b). In this study, stomatal closure observed in UE appeared to be insufficient to delay wilting symptoms. Overexpression of GhERF38, a homolog to PhERF039, decreased water deficit tolerance in Arabidopsis because the stomatal movement was insensitive to ABA treatment (Ma et al., 2017). Therefore, PhERF039 may be involved in water deficit response mechanisms regarding stomatal movements, possibly as a negative regulator in stomatal closure. 


\subsection{Conclusion}

In this study, the petunia PhERF039, a gene encoding AP2/ERF, was characterized under water deficit stress. Transgenic plants under-expression line of PhERF039 promoted outgrowth of axillary shoot and delayed flowering time. Down-regulation of PhERF039 delayed substrate drying by limiting transpirational water loss with low gs, suggesting its negative role in stomatal

closure. However, there was no visual phenotype in relation to the water deficit stress. It can't be ruled out the possibility that the effect of down-regulation of PhERF039 could have been compensated by the gene redundancy and/or functional redundancy. Further research with multiple transgenic lines (over- and under-expression) is needed to characterize the function of PhERF039 on stress responses, hormone networks, stomatal development and closure, and plant morphology. 
AtERF 35

SIERF038-LIKE

CaERF 034-LIKE

PhERF039

NtERF 039-LIKE

AtERF 38

GhERF 38

AtERF 35

SIERF038-LIKE

CaERF 034-LIKE

PhERF039

NtERF039-LIKE

AtERF 38

GhERF 38

AtERF 35

SIERF 038-LIKE

CaERF 034-LIKE

PhERF039

NtERF 039-LIKE

AtERF 38

GhERF 38

AtERF 35

SIERF038-LIKE

CaERF 034-LIKE

PhERF039

NtERF 039-LIKE

AtERF 38

GhERF 38

AtERF 35

SIERF 038-LIKE

CaERF 034-LIKE

PhERF039

NtERF 039-LIKE

AtERF 38

GhERF 38

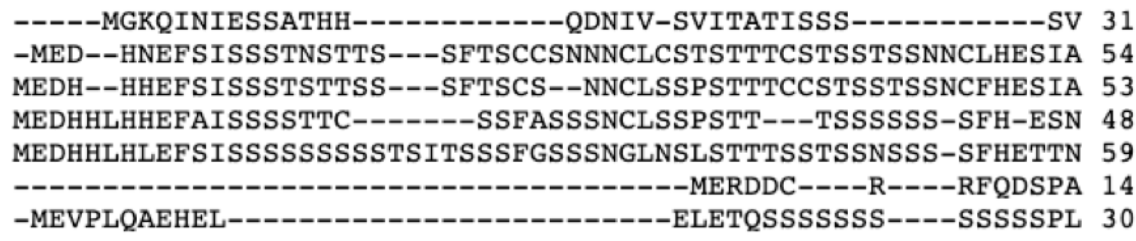

VTSSSDSWSTS-K------RSLVQDNDS-----GGKRRKSNVSDDNKNPTSYRGVRMRSW 79 KTSSNDHYKCKSKKS----AK--KNQDGNEEEDNTKIRRKKQCVVDEKHPTYRGVRKRNW 108 KSTSNCKSKKSSKKVDQDFTK--NNGNCHGNEDNKKIRRKIEGGVDEKHPTYRGVRKRNW 111 AKTPSKDYNCKSKKSSKKVQEFSNNGNEEN---K---KRRKNEGGDEKHPTYRGVRKRNW 102 AKTPSNGYNCKYKKSSKKVQDFTKNGNGHGIEEN---KKKKKNQGDEKHPTFRGVRKRNW 116 QTTE---------------RRVKYKPK------KKRAKDDDDEKVVSKHPNFRGVRMRQW 53 VIKSNK------------IQKLTQKTN------ENKRKFNNGDELEREHPTYRGVRMRQW 72

$$
\text { . . : : : }: \underline{: \star \star \star \star \star \star *}
$$

GKWVSEIREPRKKSRIWLGTYPTAEMAARAHDVAALAIKGNSGFLNFPELSGLLPRPVSC 139 GKWVSEIRQPRKKSRIWLGTYPTAEMAARAHDVAALAIKGDSAYLNFPHLADQLPRPASA 168 GKWVSEIRQPRKKSRIWLGTYPRAEMAARAHDVAALAIKGNSAYLNFPHLADQLPRPAST 171 GKWVSEIRQPRKKSRIWLGTYPTAEMAARAHDVAALAIKGHSAYLNFPHLADQLPRPASN 162 GKWVSEIRQPRKKSRIWLGTYPTAEMAARAHDVAALAIKGNSAYLNFPHLADQLPRPATT 176 GKWVSEIREPKKKSRIWLGTFSTAEMAARAHDVAALAIKGGSAHLNFPELAYHLPRPASA 113 GKWVSEIREPRKKSRIWLGTFPTAEMAARAHDVAALAIKGKSAYLNFPDIAHELPRPVSS 132

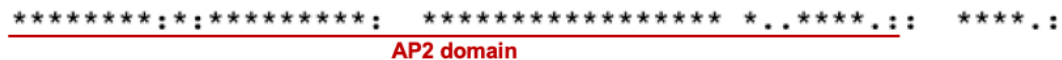

SPKDIQAAATKAAEATTWHKPVIDKKLADELSHSELLSTAQSSTSSSFVFSSDTSETSST 199 SPKDIQAAAAKAAAAS IFLEENSVE---S----SQLT-GLHSSHSSTNLASDNVQELLNS 220 SPKDIQAAAAKAAAASTFLEENSIE---S----SQLT-ELHSSHSSTNLASQNTQGLLNS 223 SPKDIQVAAAKAAT-ATVLEENFIG---GEVE-SSQI-ELHTSYSSTNLASENIQESLNS 216 SPKDIQAAAAKAAA-STFLEENSLR---DEAE-SSQI-KLHNSHSSTNLATENAQELLNS 230 DPKDIQAAAAAAAAAVAIDMDVETS-------------------SPSPSPTVTETSSPA 153 SPKDIQAAAAKAAALSYDQMAPRSP-----------------SCTASSTVSHDSTSS 172 $. * \star * * * . * *$ : ** $*$ :

DKESNEETVFDLPDLFTDGLMNPNDAFCLCNGTFTWQ-LYGEEDVGFRFEEPFNWQND 256 PSMDHDDPFFDLPDLIIDRLDQN-DRY--QYPVSTWQ-LAGADSGMFRLDEPFLWECY $\quad 274$ PSMDHDDTFFDLPDLTIDRVDQS-DRY--EYPISTWQ-LAGADSGLFRLEEPFLWECY 277 PSLDHDDTFFDLPDLSIDRVDQN-DRY--QYPTSTWQALVGADNGIFRLEEPFLWECY 271 PSMDHDDTFFDLPDLTIDQVDQN-DRY--QYPISTWQ-LAGADSGVFRLEEPFLWECY 284 MIALSDDAF SDLPDLLLNVNHNI-DGF--------WD---SFPY-----EEPFLSQSY 194 SSNDKDDAFIDLPDLLMDMNHQM-YEF--------WG---VDINGFLGSEEPRLWEYC 218 : : . $* \star * *:$ : : $\quad * \quad$ : $\quad$ :

Fig. 5.1. Comparison of deduced amino acid sequences of ERF family proteins that have high sequence similarity with PhERF039. The seven ERFs were Arabidopsis thaliana (AtERF38 and AtERF35), Solanum lycopersicum (tomato, SlERF038-like), Capsicum annuum (pepper, CaERF034-like), Petunia $\times$ hybrida (petunia, PhERF039), Nicotiana tabacum (tobacco, NtERF039-like), and Gossypium hirsutum (cotton, GhERF38). Amino acid sequences were aligned with Clustal W multiple sequence alignment. The red line under the sequences represents the highly conserved DNA-binding domain (AP2 domain). Sequence similarity is indicated by the following symbols: $(*)$, positions which have a single and fully conserved residue; $(:)$, conservation between groups of strongly similar properties; (.), conservation between groups of weakly similar properties. 


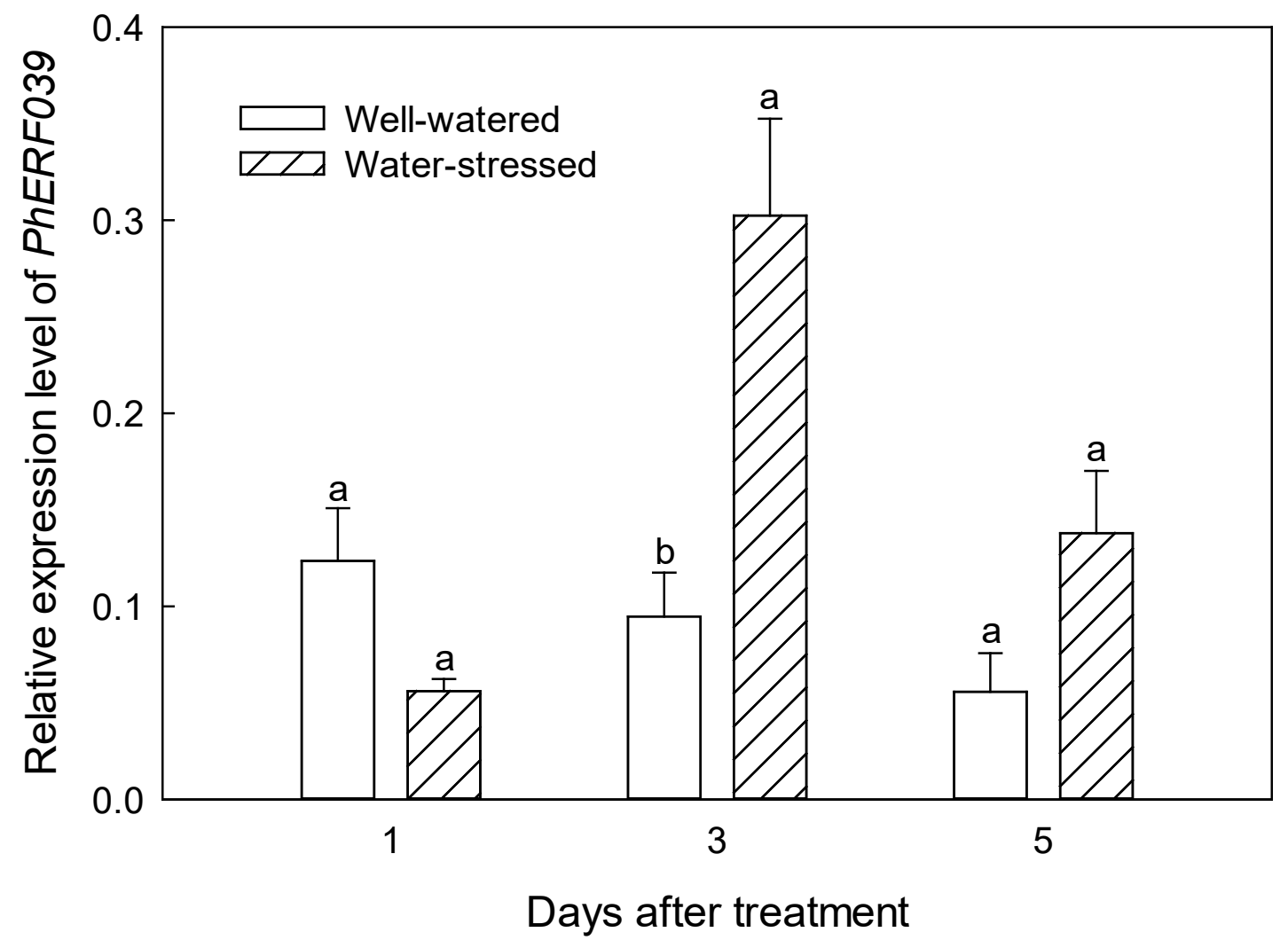

Fig. 5.2. Expression levels of PhERF039 under water deficit stress. Plants were subjected to well-watered or water deficit stress by withholding watering. Vertical bars are standard errors of the means with three replications $(n=3)$. Same letters indicated no statistical difference in each day by Tukey's test at $P \leq 0.05$. 
A
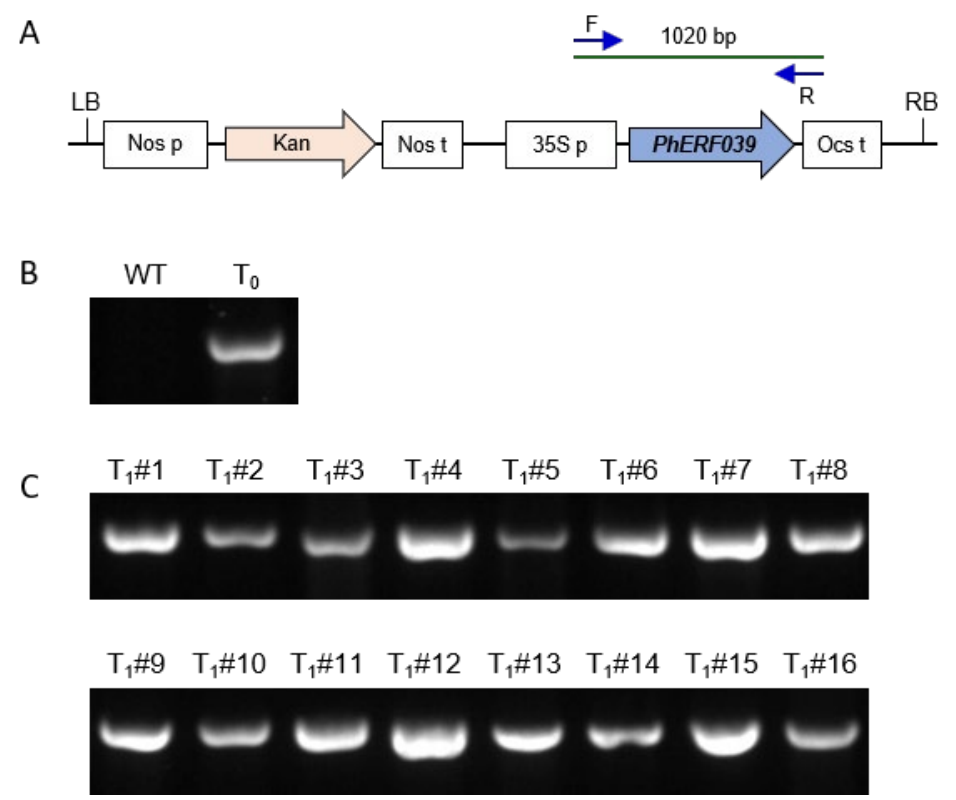

Fig. 5.3. Confirmation of PhERF039 gene integration by PCR. The diagram shows a construct used for Agrobacterium-mediate transformation of the PhERF039 (A). The bands of the PCR fragment indicate the presence of the transgene $(1020 \mathrm{bp})$ in wide type (WT) and one transgenic line $\left(\mathrm{T}_{0}\right)(\mathrm{B})$, and 16 of $\mathrm{T}_{1}$ generation $(\mathrm{C})$. The verification primer set was $5^{\prime}$ GAGGACACGCTCGAGTATAA-3' and 5'-GTAAGGATCTGAGCTACACATG-3'. 


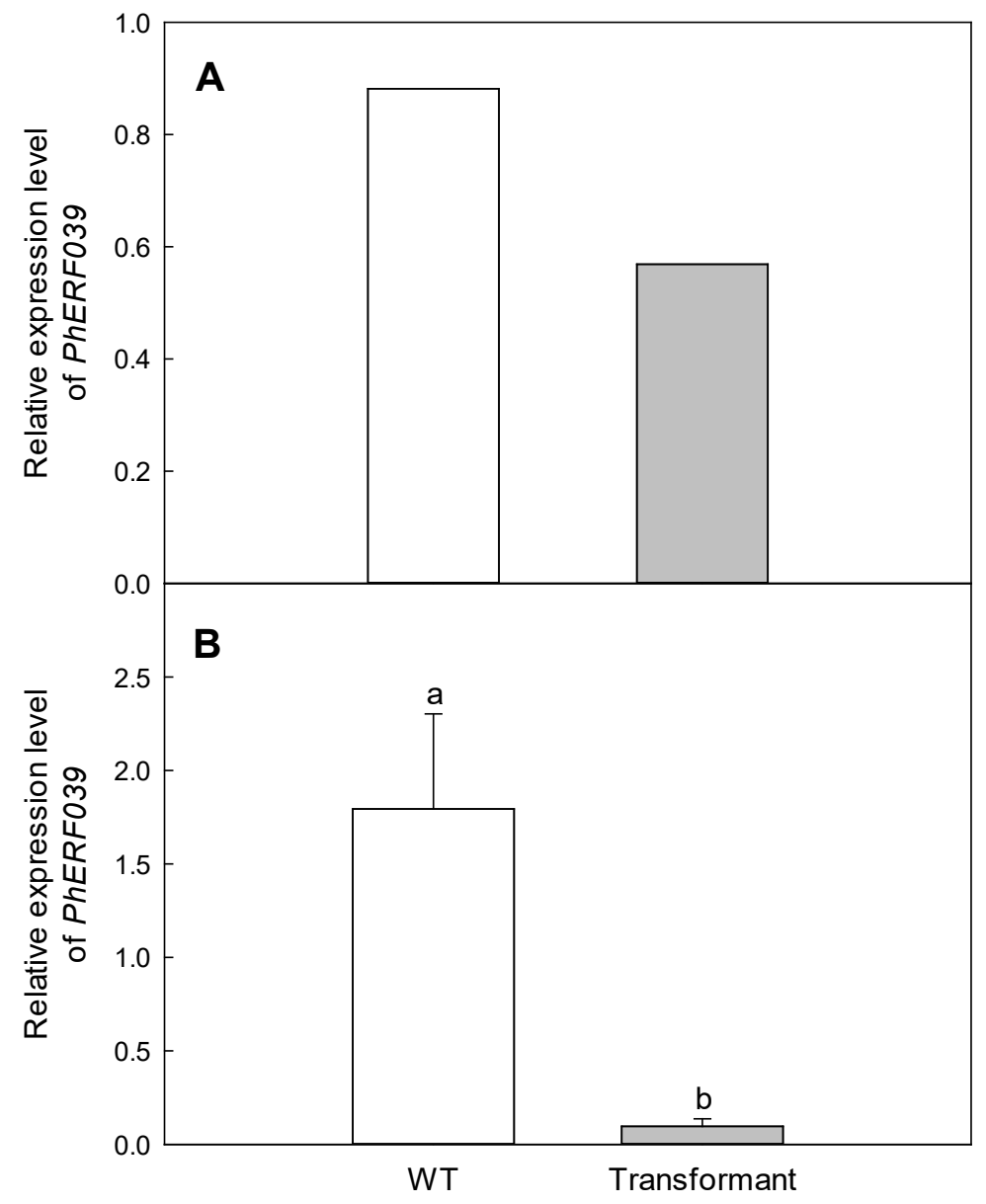

Fig. 5.4. Expression levels of PhERF039 in wild type (WT) and one transgenic line $\mathrm{T}_{0}(\mathrm{~A})$ and $\mathrm{T}_{1}(\mathrm{~B})$. The vertical bars are standard errors of the means with four replications $(\mathrm{n}=4)$. Different letters indicated statistical difference by Tukey's test at $P \leq 0.05$. 

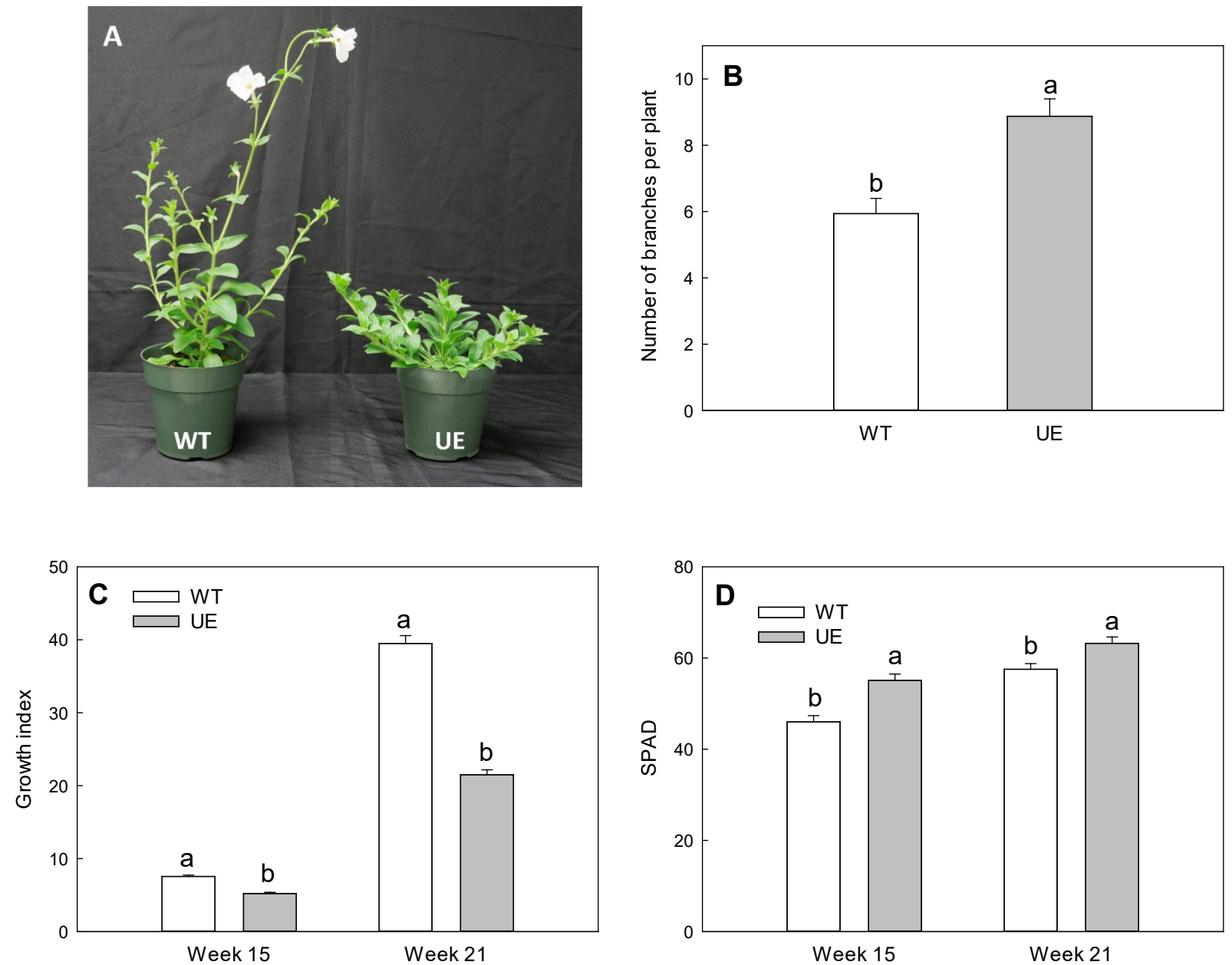

Fig. 5.5. Plant growth and development of wild type (WT) and the transgenic PhERF039 (UE; under-expression). Data were collected from $\mathrm{T}_{1}$ transgenic PhERF039. The images of WT and transgenic petunia (A) was taken at week 21, and the number of branches (B) was counted at week 21. Growth index (C) was calculated at weeks 15 and 21 by the following equation: $\mathrm{GI}=$ $[\{($ canopy widest width + perpendicular width $) / 2\}+$ height $] / 2$. SPAD (D) was measured by a SPAD-502 Chlorophyll Meter at weeks 15 and 21. Vertical bars are standard errors of the means with two replications $(n=2)$. Plants were blocked in two growth chambers and each chamber had two plants per treatment. Same letters indicated no statistical difference by Tukey's test at $P$ $\leq 0.05$. Statistical analyses were performed independently at week 15 and 21 (C and D). 

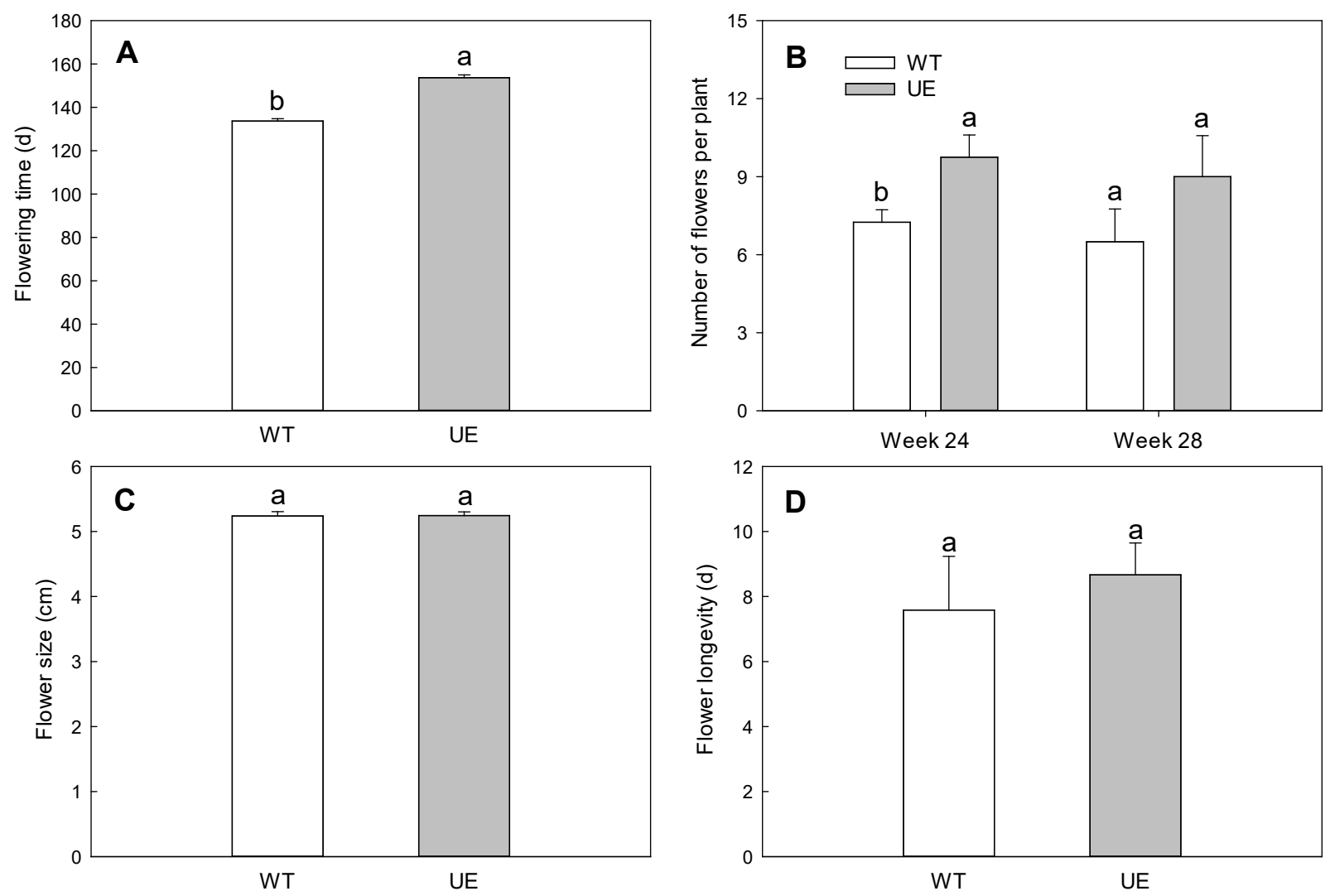

Fig. 5.6. Flower development of wild type (WT) and the transgenic PhERF039 (UE; underexpression). Data were collected from $\mathrm{T}_{1}$ transgenic PhERF039. Flowering time (A), the number of flowers (B), flower size (C), and flower longevity (D) were taken before water deficit treatment. The flowering time was determined as the number of days from the sowing date until the first flower was fully opened. The flower longevity was recorded as the time from anthesis until corolla was completed wilted. Flower size and longevity were measured at week 21 . Vertical bars are standard errors of the means with two replications $(n=2)$. Plants were blocked in two growth chambers and each chamber had two plants per treatment. Same letters indicated no statistical difference) by Tukey's test at $P \leq 0.05$. Statistical analyses were performed independently at week 24 and 28 (B). 


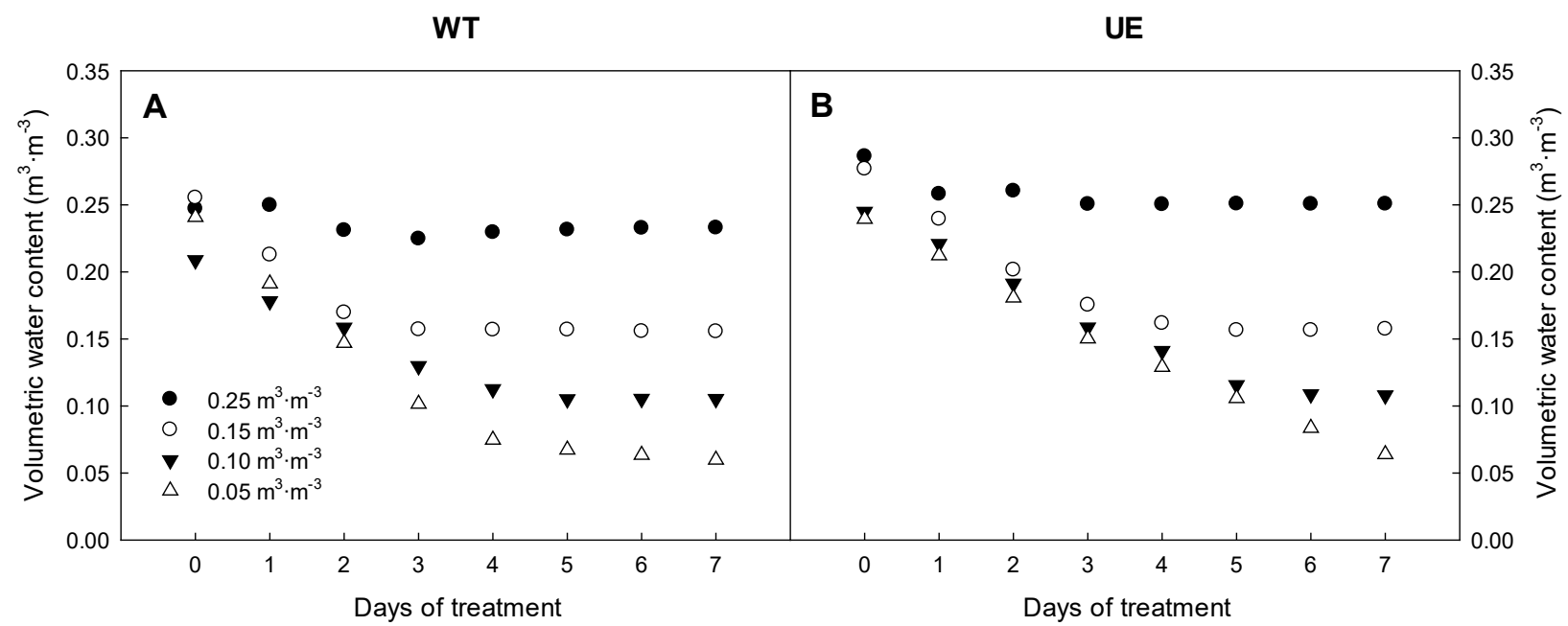

Fig. 5.7. Daily average substrate volumetric water content throughout the water deficit period for wild type (WT, A) and the transgenic PhERF039 (UE; under-expression, B). 


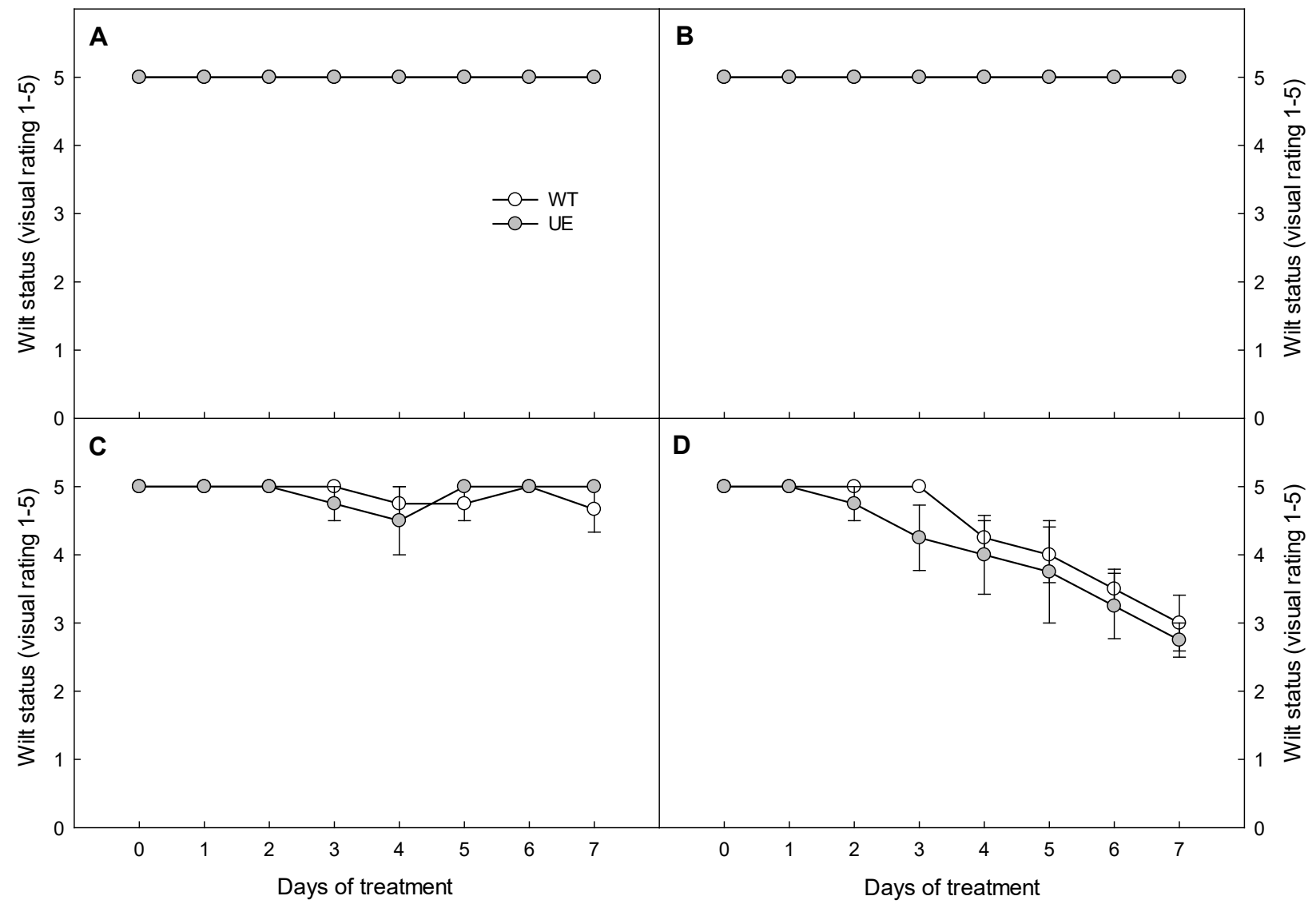

Fig. 5.8. Wilt status rating of wild type (WT) and the transgenic PhERF039 (UE; underexpression) at various volumetric water contents: 0.25 (A), $0.15(\mathrm{~B}), 0.10$ (C), and 0.05 (D) $\mathrm{m}^{3} \cdot \mathrm{m}^{-3}$. Wilt status ratings were from 5 to 1 , where $5=$ completely turgid, $4=$ soft to touch but still upright, $3=$ starting to wilt, $2=$ severely wilted, and $1=$ wilted to the point that leaves are desiccated. Vertical bars are standard errors of the means with two replications $(n=2)$. Plants were blocked in two growth chambers and each chamber had two plants per treatment. 


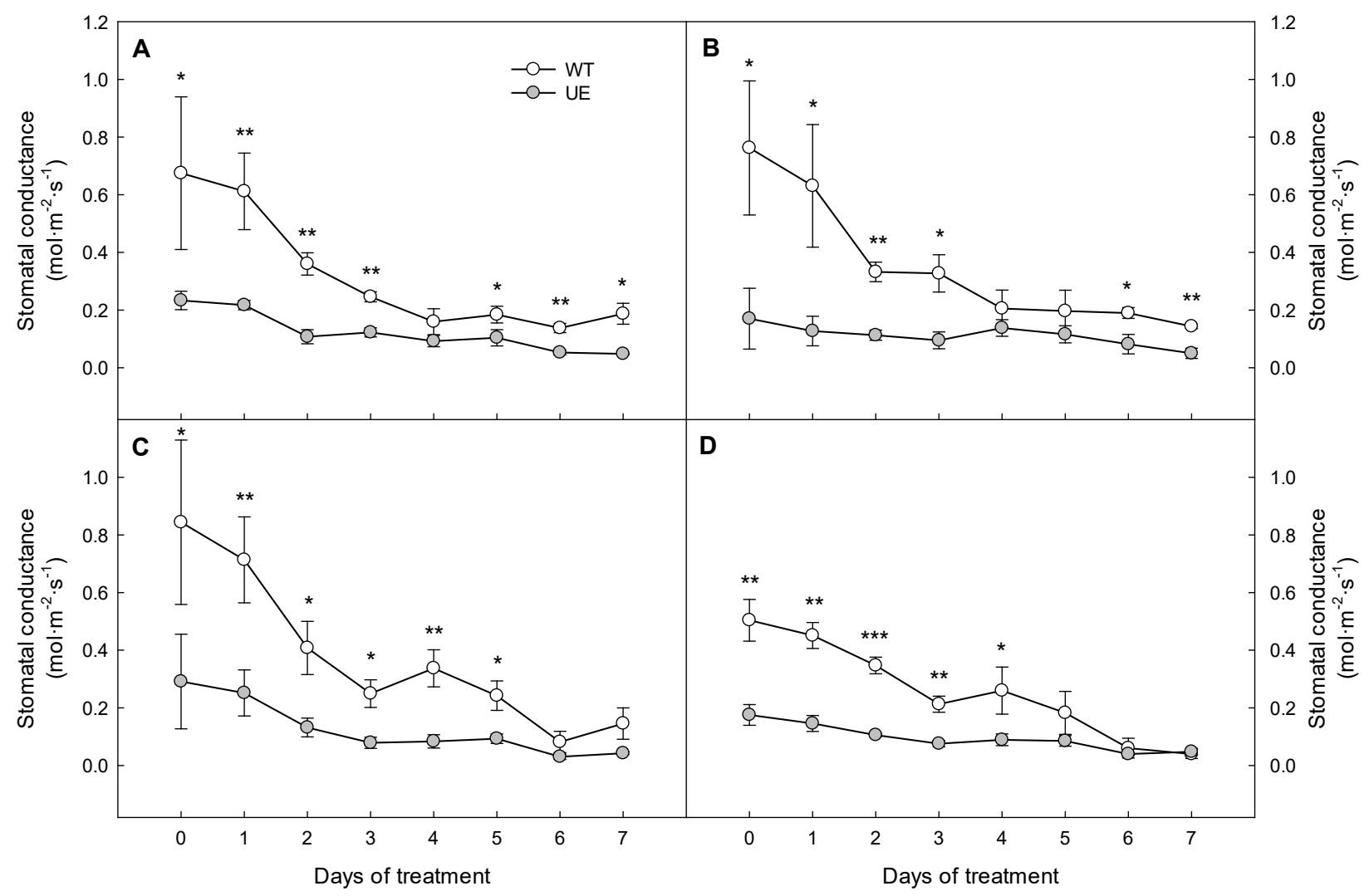

Fig. 5.9. Stomatal conductance ( $g \mathrm{~s}$ ) of wild type (WT) and the transgenic PhERF039 (UE; under-expression) at various volumetric water contents: 0.25 (A), 0.15 (B), 0.10 (C), and 0.05 (D) $\mathrm{m}^{3} \cdot \mathrm{m}^{-3}$. Stomatal conductance was measured by a LI-6400XT. Vertical bars are standard error of the means with two replications $(\mathrm{n}=2)$. Plants were blocked in two growth chambers and each chamber had two plants per treatment. ${ }^{*}, * *, * * *$ Significant at $P \leq 0.05,0.01$ or 0.001 , respectively. 


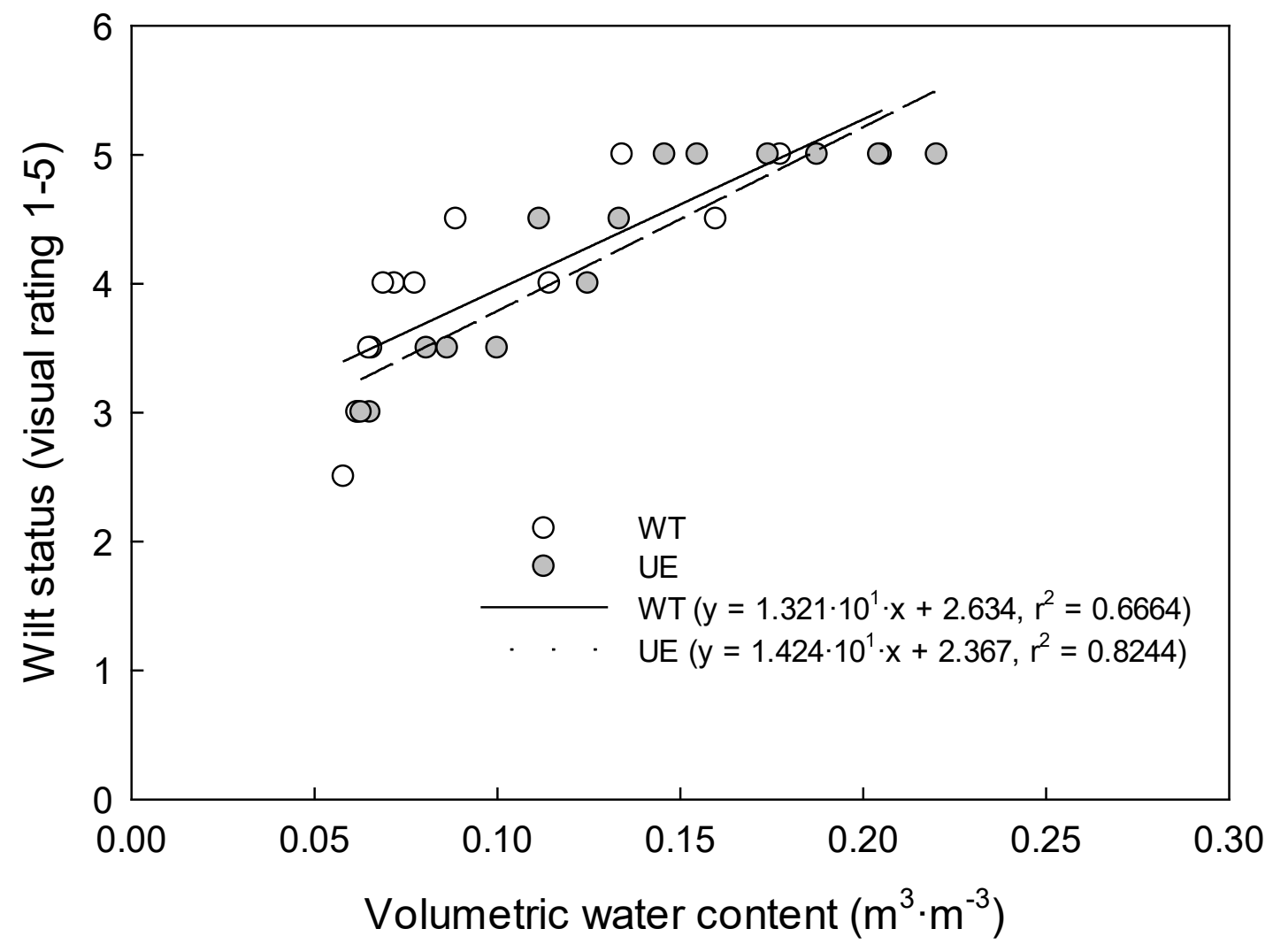

Fig. 5.10. Relationship between volumetric water content in the substrate and stomatal conductance in wild type (WT) and the transgenic PhERF039 (UE; under-expression) under treatment at $0.05 \mathrm{~m}^{3} \cdot \mathrm{m}^{-3}$. 


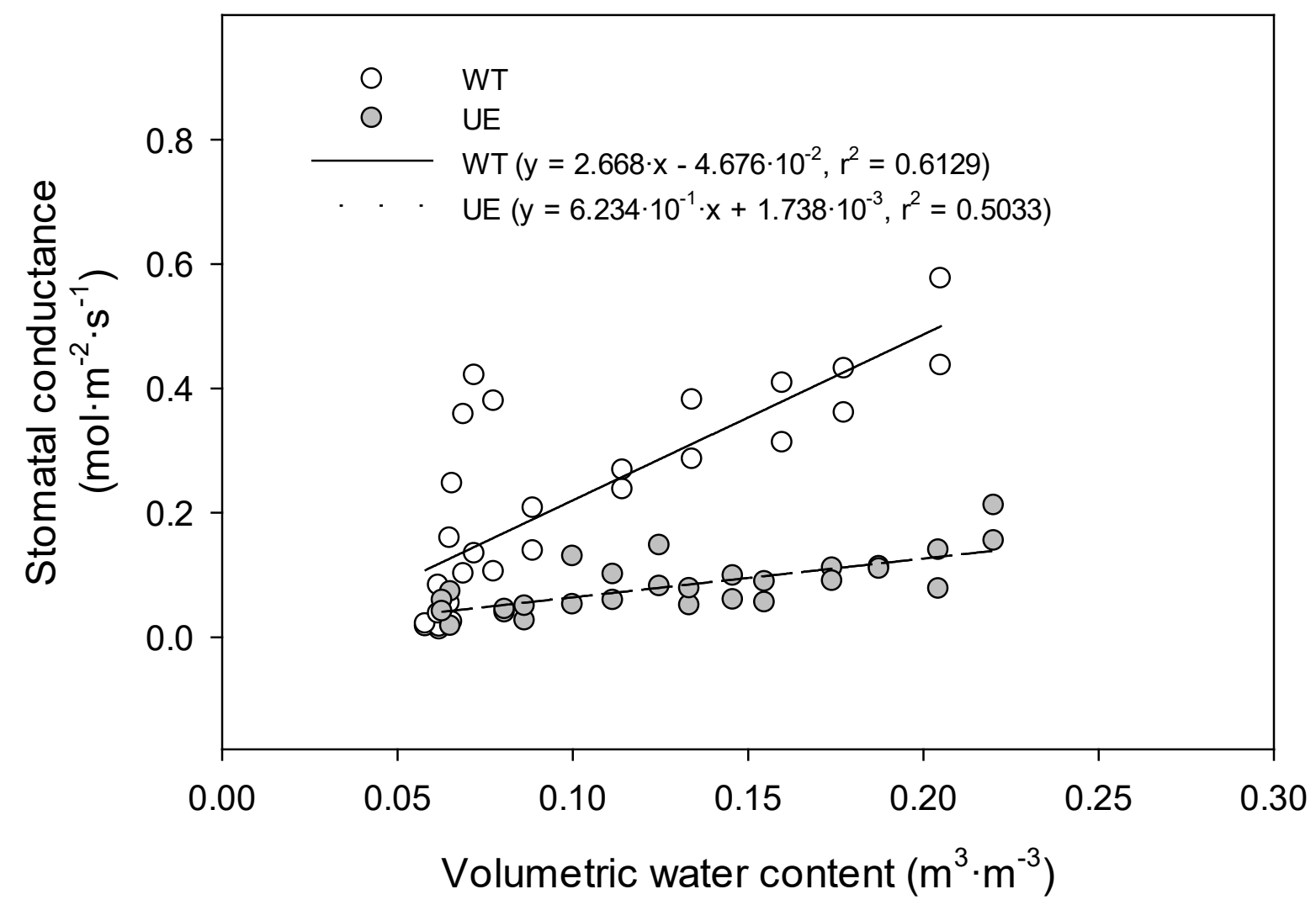

Fig. 5.11. Relationship between volumetric water content and stomatal conductance in wild type (WT) and the transgenic PhERF039 (UE; under-expression) under volumetric water content at $0.05 \mathrm{~m}^{3} \cdot \mathrm{m}^{-3}$. Stomatal conductance was measured by a LI-6400XT. 


\section{Chapter 6 Discussion}

Water deficit stress presents a considerable threat to plant growth and development, and most of crops are susceptible to water deficit, leading to a significant decrease in crop yield (Farooq et al., 2009). Floriculture crops like other crops are seriously affected by water deficit stress. They are often exposed to adverse environments during shipping and/or retailing such as high temperature and irregular irrigation, consequently resulting in water deficit stress (Ferrante et al., 2015). These unfavorable conditions are estimated to cause crop loss up to $20 \%$ of unsalable crops (Healy, 2009). The goals of this dissertation were to enhance water deficit tolerance in floriculture crops by employing physical and physiological technologies and to investigate plants' response mechanism to water deficit stress at the molecular level. The specific objectives were 1) to evaluate commercial antitranspirants for enhancing temporary water deficit tolerance in bedding plants, 2) to examine the effect of osmotic treatments as a new antitranspirant to enhance water deficit tolerance in viola, 3) to analyze transcriptomic data under water deficit in petunia using RNA sequencing, and 4) to conduct functional analysis of PhERF039 in petunia under water deficit stress.

Plants have evolved adaptation mechanisms to cope with water deficit stress. One of the mechanisms is to close stomata in response to water deficit to actively limit transpirational water loss. Utilizing such plant's response mechanism, antitranspirants can be applied to agricultural crops to limit transpirational water loss by physically blocking stomata or physiologically induce stomatal closure (Anderson and Kreith, 1978; Odlum and Colombo, 1987; Shinohara and Leskovar, 2014; Waterland et al., 2010a). In this study, five antitranspirants were evaluated to enhance temporary water deficit stress tolerance in bedding plants. One physical [ $\beta$-pinene polymer $(\beta \mathrm{P})$ ] and one physiological [a biological active form of abscisic acid (s-ABA)] antitranspirants delayed plant wilting and enhanced water deficit tolerance in bedding plants. A sharp decline of stomatal conductance was observed four hours after application of the antitranspirants. The rapid reduction in stomatal conductance might have allowed plants to maintain high water status under water deficit by preventing transpirational water loss. In contrast, another physical antitranspirant [(vinyl-acrylic polymer (VP)] and two physiological antitranspirants [sugar alcohol-based compounds (SACs)] did not show any effect on extension of shelf life. Efficacy of physical antitranspirants are dependent on chemical's adhesion and retention on leaves (Palliotti et al., 2010; Pathan et al., 2009). VP might not be an effective 
antitranspirant to coat the stomata and retain on the leaf surface in the tested cultivars. SACs are supposed to reduce water potential in growing media in order to mimic water deficit stress and induce stomatal closure. SACs failed to reduce water potential in substrate and trigger water stress responses even at twice the recommendation rate. Among five antitranspirants, applications of $\beta \mathrm{P}$ and s-ABA were efficient to delay plant wilting; however, $\beta \mathrm{P}$ caused floral damage in all tested plants and s-ABA has frequently been mentioned to cause foliar chlorosis and leaf abscission as side effects (Kim and van Iersel, 2011; Waterland et al., 2010a; Weaver and van Iersel, 2014).

The application of antitranspirants yielded a limited success in enhancing water deficit tolerance. To develop a more effective technology with minimal damage to plants, the feasibility of osmotic treatments was examined in viola. Among seven chemicals, $\mathrm{CaCl}_{2}$ was the most effective agent at delaying wilting and enhancing water deficit tolerance without any visual side effects. The reduction in stomatal conductance with $\mathrm{CaCl}_{2}$ treatment appeared to help viola to maintain high leaf relative water content at the initial stage of water deficit stress, resulting in extended shelf life under water deficit. Exogenous calcium treatments have been demonstrated to enhance tolerance to abiotic and biotic stresses in several crops by producing antioxidants and osmolytes (Jaleel et al., 2007; Xu et al., 2013a). An appropriate concentration of $\mathrm{CaCl}_{2} \mathrm{treatment}$ could be a useful method to enhance water deficit tolerance by inducing stomatal closure in advance to water deficit and probably by maintaining cell stability with antioxidants and osmoprotectants. In addition to rapid stomatal closure, an earlier stress, herein osmotic treatments, may help plants to respond more quickly and actively to later stresses, which is known as priming (Savvides et al., 2016). Primed plants tend to have higher level of antioxidants, osmolytes, and upregulated expression of stress responsive genes, showing enhanced tolerance to later stresses (Hossain et al., 2017; Li and Liu, 2016). Osmotic treatments with high concentration of chemicals, such as fertilizers, could be a practical method to enhance water deficit tolerance.

Based on the results of antitranspirant and osmotic treatments, closing stomata was an effective strategy to increase water deficit stress tolerance in some bedding plants. To investigate the water deficit stress response mechanisms at the molecular level, transcriptome analysis was performed in petunia under water deficit. Transcriptome data showed that thousands of genes were differentially expressed at the early stage of water deficit. Among them, genes known to be 
associated with the redox homeostasis using sulfur metabolism were enriched, suggesting the involvement of antioxidant activity to prevent cell damages caused by reactive oxygen species and to maintain cell stability. In addition, genes encoding components of abscisic acid (ABA) biosynthesis and signal transduction pathways were differentially expressed under water deficit. ABA biosynthesis genes (NCEDs and $A A O)$ and ABA degradation gene (CYO707A) were downregulated, indicating that the inhibition of ABA degradation triggered water deficit tolerance in petunia rather than the promotion of ABA synthesis (Takeuchi et al., 2016). Transcription changes of ABA signal transduction occurred in a timely manner. At the immediate stage of water deficit, the ABA receptor, PYL, was upregulated followed by upregulated expression of next components, PP2C and ABF. Ethylene was also shown to be involved in water deficit tolerance in petunia. Two ethylene biosynthesis enzymes (SAMS and ACO) were upregulated, suggesting the promotion of ethylene production in petunia under water deficit. Increased expression of ethylene signaling components (ETR1 and EIN3) was observed at the early stage of water deficit, and especially, EIN3 is known to induced ERF1 which is a major TF in abiotic and biotic stress responses (Müller and Munné-Bosch, 2015). These results suggested that ABA and ethylene biosynthesis and signal transduction play important roles in the early responses to water deficit in petunia.

Transcriptomic results showed that 107 transcription factors (TFs) of 34 families were differentially expressed under water deficit. Previous researches have documented that a wide range of TFs play as major regulators in abiotic and abiotic stress responses and they could be candidates to improve stress tolerance in plants (Joshi et al., 2016; Mizoi et al., 2012; Xie et al., 2019). A large portion of TFs in plant stress response are classified into members of AREB/ABF, AP2/ERF, MYB, WRKY, NAC, and bZIP TF families (Joshi et al., 2016). In this research, AP2/ERF were most dominant TF family under water deficit, and one of them, PhERF039, were selected for functional analysis. A homolog gene, GhERF38, was reported to be induced by salt, water deficit stresses, and ABA treatment (Ma et al., 2017). The expression of PhERF039 was upregulated at the early stage of water deficit. The function of PhERF039 was evaluated at four volumetric water contents maintained by an automatic irrigation system. The overexpression line of PhERF039 turned out to be an RNAi line. Overall, downexpression of PhERF039 delayed plant wilting, exhibited low stomatal conductance, and reached the set volumetric water content of soilless media slowly. Overexpression of GhERF38, a homolog of 
PhERF039, reduced salt and water deficit stress tolerance due to ABA-insensitive stomatal movements and large stomatal aperture (Ma et al., 2017). This result indicated that PhERF039 could play a negative role in stomatal closing and water deficit tolerance. The low stomatal conductance might result from induction of stomatal closure and/or low stomatal density. Arabidopsis applied with ethylene precursor (ACC) increased stomatal density, while impaired ethylene signaling pathways reduced stomatal density, suggesting ethylene plays a positive role in stomatal development (Serna and Fenoll, 2001). Suppression of PhERF039 also promoted outgrowth of branching and delayed flowering. Further research with additional lines of overand under-expression lines is needed to identify the exact roles of PhERF039 in stomatal closure, density and any other phenotypes.

Transcriptomic data revealed that genes regarding ethylene biosynthesis ( $S A M S$ and $A C O$ ) were induced under water deficit, suggesting increased ethylene production may play as a signal moderator in water deficit responses. Additionally, the osmotic treatment with calcium enhanced water deficit stress tolerance. It has been reported that ethylene and calcium are signal modulators in abiotic stress responses (Ranty et al., 2016; Tao et al., 2015), and crosstalk between ethylene and calcium has been found in plants (Li et al., 2018a; Raz and Fluhr, 1992; $\mathrm{Xu}$ and Zhang, 2014; Yu et al., 2019). The application of $\mathrm{Ca}^{2+}$ promoted the ethylene production by increasing the transcript levels of ethylene synthesis genes, $A C S \mathrm{~s}$ and $A C O \mathrm{~s}$, and induced adventitious root formation in Cucumis sativus under salt stress (Yu et al., 2019). Li et al. (2018a) found that calcium-dependent protein kinase 5 (CPK5) and CPK6 regulated the expression of $A C S$ genes and increased ethylene content in response to wounding in Arabidopsis. These results indicated that calcium was involved in ethylene production under stress conditions, and $\mathrm{Yu}$ et al. (2019) suggested that the $\mathrm{Ca}^{2+}$ might act as upstream of ethylene signaling pathways under salt stress. However, the crosstalk between ethylene and calcium under water deficit stress is still unknown.

In conclusion, antitranspirants and osmotic treatments were efficient methods to enhance temporary water deficit tolerance in floricultural crops. Especially, the osmotic treatment including priming could be further developed to be a practical tool to increase water stress tolerance with virtually no damage to the plants. Time-course transcriptomic analysis not only allowed us to identify candidate genes at the early stage of water deficit for functional analyses but also provided an insight into how some of the genes involved in homeostasis, hormone 
signaling, and transcription factors could play an important role during water deficit stress. An AP2/ERT TF, PhERF039, might be involved in water deficit stress responses, especially regulation of transpirational water loss. Results from this research will help us to advance our understanding of plant responses to water deficit stress and enhance temporary water deficit tolerance in floriculture crops. 


\section{Supplementary materials}

Supplementary Table S1. Primers used for qPCR analyses.

\begin{tabular}{llc}
\hline Gene ID & Primer sequence & Product length (bp) \\
\hline Internal control $(P h E F 1 \alpha)$ & $\begin{array}{l}\text { F: CCTGGTCAAATTGGAAACGG } \\
\text { R: CAGATCGCCTGTCAATCTTGG }\end{array}$ & 103 \\
\hline TRINITY_DN28967_c0_g1_i1 & $\begin{array}{l}\text { F: AGTGACAGGACCTTGGTAGA } \\
\text { R: CCACAGGGATTTCTCCGATATT }\end{array}$ & 107 \\
\hline TRINITY_DN30261_c0_g1_i1 & $\begin{array}{l}\text { F: GAAGCTGAAGGAGACCACTAAC } \\
\text { R: TACTCCATGAGAAAGACAGTGAATAC }\end{array}$ & 103 \\
\hline TRINITY_DN27512_c3_g1_i2 & $\begin{array}{l}\text { F: TGACCGTTATCAATACCCAACTT } \\
\text { R: CCACTAATAGCACTCCCACAAA }\end{array}$ & 104 \\
\hline TRINITY_DN25243_c1_g2_i1 & $\begin{array}{l}\text { F: GGTCCTGTTAGCTTGCTACATT } \\
\text { R: CATAAGGAGCCCAAAGAGAATCA }\end{array}$ & 103 \\
\hline TRINITY_DN26771_c1_g1_i1 & $\begin{array}{l}\text { F: AATCTGAGCCGTTGGATTCAG } \\
\text { R: GTGGTTTACTGGGACCACTTT }\end{array}$ & 110 \\
\hline
\end{tabular}




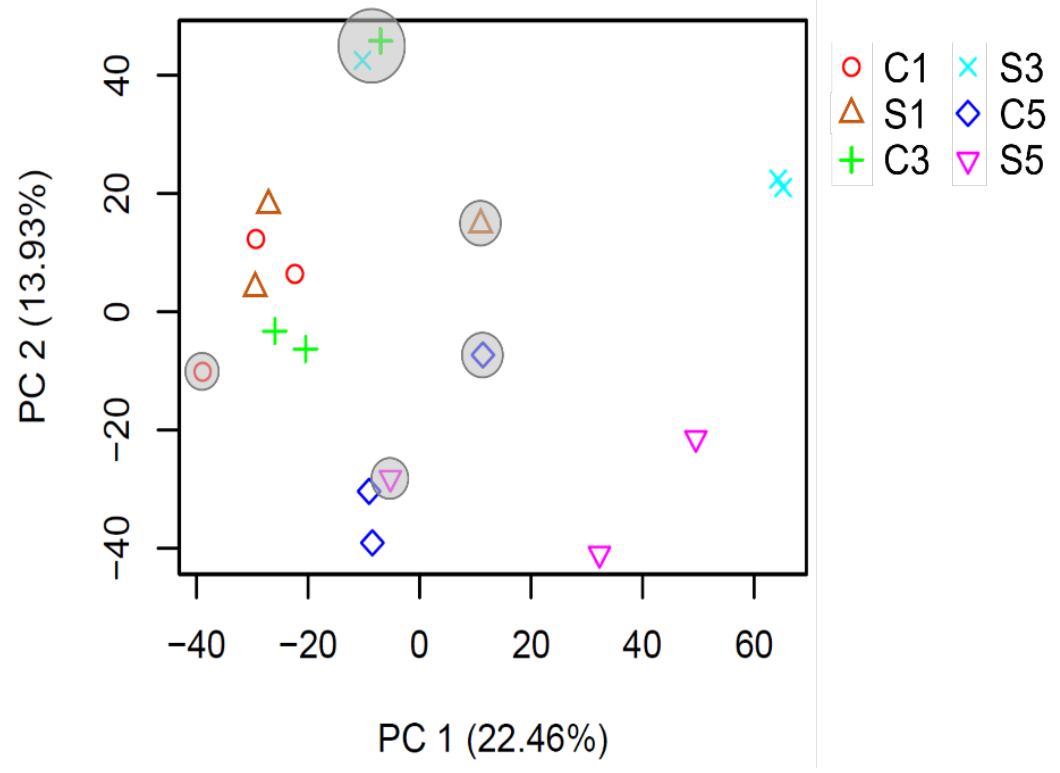

Supplementary Fig. S1. Principle component analysis plot of the RNA-seq libraries. C and S correspond to control and stressed plants, respectively, and the numbers indicate the number of days after withholding water. Gray-circled samples are considered as outliers, and they were excluded for further analyses. 
Supplementary Table S2. The number of sequenced, trimmed, and mapped reads (50 bp-paired end).

\begin{tabular}{|c|c|c|c|c|c|}
\hline Day & Treatment & Rep. & No. of reads & $\begin{array}{l}\text { No. of reads after } \\
\text { trimmed }\end{array}$ & No. of reads mapped \\
\hline \multirow[t]{6}{*}{ Day 1} & Control & 1 & $11,705,939$ & $11,669,651$ & $10,089,705(86.5 \%)$ \\
\hline & & 2 & $12,639,684$ & $12,592,917$ & $11,014,104(87.5 \%)$ \\
\hline & & 3 & $16,604,788$ & $16,543,350$ & $14,679,300(88.7 \%)$ \\
\hline & Stressed & 1 & $15,116,700$ & $15,071,350$ & $13,311,211(88.3 \%)$ \\
\hline & & 2 & $16,083,109$ & $16,023,601$ & $14,214,999(88.7 \%)$ \\
\hline & & 3 & $17,698,038$ & $17,639,634$ & $15,333,796(86.9 \%)$ \\
\hline \multirow[t]{6}{*}{ Day 3} & Control & 1 & $18,128,347$ & $18,061,272$ & $15,529,575(86.0 \%)$ \\
\hline & & 2 & $12,954,123$ & $12,910,079$ & $10,959,056(84.9 \%)$ \\
\hline & & 3 & $14,756,225$ & $14,683,919$ & $12,845,284(87.5 \%)$ \\
\hline & Stressed & 1 & $15,778,128$ & $15,707,126$ & $13,827,321(88.0 \%)$ \\
\hline & & 2 & $11,907,904$ & $11,857,891$ & $10,196,479(86.0 \%)$ \\
\hline & & 3 & $14,329,417$ & $14,274,965$ & $12,316,744(86.3 \%)$ \\
\hline \multirow[t]{6}{*}{ Day 5} & Control & 1 & $18,716,488$ & $18,662,210$ & $16,918,912(90.7 \%)$ \\
\hline & & 2 & $8,898,370$ & $8,874,344$ & $7,747,028(87.3 \%)$ \\
\hline & & 3 & $14,483,784$ & $14,438,884$ & $12,661,474(87.8 \%)$ \\
\hline & Stressed & 1 & $13,734,523$ & $13,687,826$ & $11,939,870(87.2 \%)$ \\
\hline & & 2 & $14,612,044$ & $14,565,285$ & $12,929,069(88.7 \%)$ \\
\hline & & 3 & $15,216,799$ & $15,158,975$ & $13,349,636(88.0 \%)$ \\
\hline Total & & & $263,364,410$ & $262,427,357$ & $229,863,563(87.6 \%)$ \\
\hline
\end{tabular}




\begin{tabular}{|c|c|c|c|c|c|c|c|c|c|}
\hline TF family & Contig ID & Day1 & Day3 & Day5 & TF family & Contig ID & Day1 & Day3 & Day5 \\
\hline \multirow[t]{8}{*}{ AP2/ERF } & TRINITY_DN11899_c0_g2_i1 & & & & AP2/ERF & TRINITY_DN1576_c0_g1_i1 & & & \\
\hline & TRINITY_DN14217_c0_g1_i1 & & & & & TRINITY_DN19375_c0_g1_i1 & & & \\
\hline & TRINITY_DN22124_c0_g1_i1 & & & & & TRINITY_DN22569_c2_g1_i1 & & & \\
\hline & TRINITY_DN24527_c0_g1_i1 & & & & & TRINITY_DN25527_c0_g1_i1 & & & \\
\hline & TRINITY_DN25125_c2_g1_i1 & & & & & TRINITY_DN26837_c1_g1_i1 & & & \\
\hline & TRINITY_DN30613_c0_g1_i1 & & & & & TRINITY_DN33308_c0_g1_i1 & & & \\
\hline & TRINITY_DN32633_c0_g1_i1 & & & & ARF & TRINITY_DN26137_c0_g1_i1 & & & \\
\hline & TRINITY_DN33611_c0_g1_i1 & & & & B3 & TRINITY_DN26145_c0_g2_i1 & & & \\
\hline ARF & TRINITY_DN16672_c0_g1_i1 & & & & bHLH & TRINITY_DN19460_c0_g1_i1 & & & \\
\hline ARR-B & TRINITY_DN26278_c0_g1_i5 & & & & & TRINITY_DN21443_c0_g1_i1 & & & \\
\hline B3 & TRINITY_DN16861_c0_g1_i1 & & & & & TRINITY_DN23704_c0_g1_i1 & & & \\
\hline BES1 & TRINITY_DN23555_c0_g1_i1 & & & & & TRINITY_DN8763_c0_g1_i1 & & & \\
\hline bHLH & TRINITY_DN15692_c0_g1_i1 & & & & bZIP & TRINITY_DN24061_c1_g1_i1 & & & \\
\hline \multirow[t]{4}{*}{ bZIP } & TRINITY_DN18154_c0_g1_i1 & & & & & TRINITY_DN24125_c0_g1_i1 & & & \\
\hline & TRINITY_DN23936_c2_g1_i1 & & & & & TRINITY_DN26160_c1_g1_i1 & & & \\
\hline & TRINITY_DN26771_c1_g1_i1 & & & & $\mathrm{C} 2 \mathrm{H} 2$ & TRINITY_DN19046_c0_g2_i2 & & & \\
\hline & TRINITY_DN29035_c0_g1_i1 & & & & & TRINITY_DN28250_c0_g1_i1 & & & \\
\hline \multirow[t]{6}{*}{$\mathrm{C} 2 \mathrm{H} 2$} & TRINITY_DN18638_c0_g1_i1 & & & & $\mathrm{C} 3 \mathrm{H}$ & TRINITY_DN26470_c2_g1_i2 & & & \\
\hline & TRINITY_DN18857_c0_g1_i1 & & & & FAR1 & TRINITY_DN26114_c0_g1_i1 & & & \\
\hline & TRINITY_DN22404_c0_g1_i1 & & & & G2-like & TRINITY_DN24859_c1_g1_i1 & & & \\
\hline & TRINITY_DN25252_c1_g1_i1 & & & & GATA & TRINITY_DN3858_c0_g1_i1 & & & \\
\hline & TRINITY_DN27512_c3_g1_i1 & & & & HD-ZIP & TRINITY_DN26880_c0_g2_i1 & & & \\
\hline & TRINITY_DN27512_c3_g1_i2 & & & & & TRINITY_DN26880_c2_g1_i1 & & & \\
\hline \multirow[t]{2}{*}{$\mathrm{C} 3 \mathrm{H}$} & TRINITY_DN20019_c0_g1_i1 & & & & LBD & TRINITY_DN1663_c0_g1_i1 & & & \\
\hline & TRINITY_DN24475_c1_g1_i1 & & & & & TRINITY_DN27302_c3_g3_i1 & & & \\
\hline CAMTA & TRINITY_DN26942_c0_g1_i1 & & & & MYB & TRINITY_DN23457_c0_g2_i1 & & & \\
\hline CPP & TRINITY_DN9774_c0_g1_i1 & & & & & TRINITY_DN26481_c1_g1_i1 & & & \\
\hline \multirow[t]{2}{*}{ Dof } & TRINITY_DN23450_c1_g1_i1 & & & & MYB_related & TRINITY_DN15219_c0_g1_i1 & & & \\
\hline & TRINITY_DN27808_c0_g1_i1 & & & & & TRINITY_DN23457_c0_g1_i1 & & & \\
\hline \multirow[t]{2}{*}{ EIL } & TRINITY_DN25243_c1_g2_i1 & & & & & TRINITY_DN3225_c0_g1_i1 & & & \\
\hline & TRINITY_DN25956_c3_g1_i1 & & & & NAC & TRINITY_DN14919_c0_g1_i1 & & & \\
\hline G2-like & TRINITY_DN30476_c0_g1_i1 & & & & & TRINITY_DN20304_c0_g1_i1 & & & \\
\hline \multirow[t]{3}{*}{ GATA } & TRINITY_DN30187_c0_g2_i1 & & & & & TRINITY_DN21562_c0_g1_i1 & & & \\
\hline & TRINITY_DN5229_c0_g1_i1 & & & & & TRINITY_DN25748_c0_g1_i1 & & & \\
\hline & TRINITY_DN6447_c0_g1_i1 & & & & & TRINITY_DN25748_c0_g1_i2 & & & \\
\hline GRAS & TRINITY_DN19810_c1_g1_i1 & & & & & TRINITY_DN25748_c0_g1_i3 & & & \\
\hline \multirow[t]{3}{*}{ GRF } & TRINITY_DN11489_c0_g1_i1 & & & & & TRINITY_DN25748_c0_g1_i4 & & & \\
\hline & TRINITY_DN25774_c0_g2_i1 & & & & NF-YC & TRINITY_DN30498_c0_g1_i1 & & & \\
\hline & TRINITY_DN7120_c0_g1_i1 & & & & Nin-like & TRINITY_DN23892_c0_g1_i1 & & & \\
\hline \multirow[t]{3}{*}{ HD-ZIP } & TRINITY_DN10248_c0_g1_i1 & & & & TCP & TRINITY_DN14692_c0_g1_i1 & & & \\
\hline & TRINITY_DN32334_c0_g1_i1 & & & & & TRINITY_DN9146_c0_g1_i1 & & & \\
\hline & TRINITY_DN7266_c0_g1_i1 & & & & WRKY & TRINITY_DN20629_c0_g1_i1 & & & \\
\hline \multirow[t]{2}{*}{ HSF } & TRINITY_DN11558_c0_g1_i1 & & & & & TRINITY_DN20910_c0_g1_i1 & & & \\
\hline & TRINITY_DN32654_c0_g1_i1 & & & & & TRINITY_DN21274_c0_g1_i1 & & & \\
\hline M-type_MADS & TRINITY_DN24234_c0_g1_i2 & & & & & & & & \\
\hline MIKC_MADS & TRINITY_DN25216_c0_g1_i1 & & & & & & & & \\
\hline MYB & TRINITY_DN34055_c0_g1_i1 & & & & Color ke & & & & \\
\hline \multirow[t]{5}{*}{ MYB_related } & TRINITY_DN14722_c0_g1_i1 & & & & & & & & \\
\hline & TRINITY_DN19690_c0_g1_i1 & & & & & & & & \\
\hline & TRINITY_DN27357_c0_g2_i1 & & & & 1 & & & & \\
\hline & TRINITY_DN31472_c0_g1_i1 & & & & $-4 \quad 0$ & 4 & & & \\
\hline & TRINITY_DN338_c0_g1_i1 & & & & $\log _{2}{ }^{\mathrm{FC}}$ & & & & \\
\hline \multirow[t]{4}{*}{ NAC } & TRINITY_DN15372_c0_g1_i1 & & & & & & & & \\
\hline & TRINITY_DN24666_c2_g1_i1 & & & & & & & & \\
\hline & TRINITY_DN25869_c1_g1_i1 & & & & & & & & \\
\hline & TRINITY_DN6165_c0_g1_i1 & & & & & & & & \\
\hline NF-YA & TRINITY_DN22148_c0_g1_i1 & & & & & & & & \\
\hline & TRINITY_DN22148_c0_g2_i1 & & & & & & & & \\
\hline NF-YB & TRINITY_DN24862_c0_g1_i1 & & & & & & & & \\
\hline TCP & TRINITY_DN10128_c0_g1_i1 & & & & & & & & \\
\hline & TRINITY_DN30301_c0_g1_i1 & & & & & & & & \\
\hline Trihelix & TRINITY_DN9852_c1_g1_i1 & & & & & & & & \\
\hline ZF-HD & TRINITY_DN2846_c0_g1_i1 & & & & & & & & \\
\hline
\end{tabular}

Supplementary. Fig. S2. Heatmap of differentially expressed TFs under water deficit.

Upregulated (left and pink) and downregulated (right and blue) TFs. 


\section{References}

Achard, P., M. Baghour, A. Chapple, P. Hedden, D. Van Der Straeten, P. Genschik, T. Moritz, and N.P. Harberd. 2007. The plant stress hormone ethylene controls floral transition via DELLA-dependent regulation of floral meristem-identity genes. PNAS 104:6484-6489.

Adrees, M., S. Ali, M. Iqbal, S.A. Bharwana, Z. Siddiqi, M. Farid, Q. Ali, R. Saeed, and M. Rizwan. 2015. Mannitol alleviates chromium toxicity in wheat plants in relation to growth, yield, stimulation of anti-oxidative enzymes, oxidative stress and $\mathrm{Cr}$ uptake in sand and soil media. Ecotoxicology Environ. Safety 122:1-8.

Agehara, S. and D.I. Leskovar. 2012. Characterizing concentration effects of exogenous abscisic acid on gas exchange, water relations, and growth of muskmelon seedlings during water stress and rehydration. J. Am. Soc. Hortic. Sci. 137:400-410.

Altschul, S.F., T.L. Madden, A.A. Schäffer, J. Zhang, Z. Zhang, W. Miller, and D. Lipman. 1997. Gapped BLAST and PSI-BLAST: a new generation of protein database search programs. Nucleic Acids Res. 25:3389-3402.

Anderson, J.E. and F. Kreith. 1978. Effects of film-forming and silicone antitranspirants on four herbaceous plant species. Plant Soil 49:161-173.

Armitage, A.M. 1993. Bedding plants: prolonging shelf performance: postproduction care and handling. Ball Publishing.

Arndt, S.K., A. Irawan, and G.J. Sanders. 2015. Apoplastic water fraction and rehydration techniques introduce significant errors in measurements of relative water content and osmotic potential in plant leaves. Physiol. Plant. 155:355-368.

Arraes, F.B.M., M.A. Beneventi, M.E.L. de Sa, J.F.R. Paixao, E.V.S. Albuquerque, S.R.R. Marin, E. Purgatto, A.L. Nepomuceno, and M.F. Grossi-de-Sa. 2015. Implications of ethylene biosynthesis and signaling in soybean drought stress tolerance. BMC Plant Biol. $15: 213$.

Astacio, M.G. and M.W. van Iersel. 2011. Determining the effects of abscisic acid drenches on evapotranspiration and leaf gas exchange of tomato. HortScience 46:1512-1517.

Bai, Z., T. Wang, Y. Wu, K. Wang, Q. Liang, Y. Pan, B. Jiang, L. Zhang, G. Liu, and Y. Jia. 2017. Whole-transcriptome sequence analysis of differentially expressed genes in Phormium tenax under drought stress. Sci. Rpt. 7:41700. 
Barghini, E., R.M. Cossu, A. Cavallini, and T. Giordani. 2015. Transcriptome analysis of response to drought in poplar interspecific hybrids. Genomics Data 3:143-145.

Bartels, D. and R. Sunkar. 2005. Drought and salt tolerance in plants. Crit Rev. Plant. Sci. 24:2358.

Basu, S., V. Ramegowda, A. Kumar, and A. Pereira. 2016. Plant adaptation to drought stress. F1000 Res. 5.

Bela, K., E. Horváth, Á. Gallé, L. Szabados, I. Tari, and J. Csiszár. 2015. Plant glutathione peroxidases: emerging role of the antioxidant enzymes in plant development and stress responses. J. Plant Physiol. 176:192-201.

Bhargava, S. and K. Sawant. 2013. Drought stress adaptation: Metabolic adjustment and regulation of gene expression. Plant Breed. 132:21-32.

Bhatnagar-Mathur, P., V. Vadez, and K.K. Sharma. 2008. Transgenic approaches for abiotic stress tolerance in plants: Retrospect and prospects. Plant Cell Rpt. 27:411-424.

Bihani, P., B. Char, and S. Bhargava. 2011. Transgenic expression of sorghum DREB2 in rice improves tolerance and yield under water limitation. J. Agr. Sci. 149:95-101.

Blanchard, M., L. Newton, E. Runkle, D. Woolard, and C. Campbell. 2007. Exogenous applications of abscisic acid improved the postharvest drought tolerance of several annual bedding plants. Acta Hort. 775:127-132.

Brault, M., Z. Amiar, A. Pennarun, M. Monestiez, Z. Zhang, D. Cornel, O. Dellis, H. Knight, F. Bouteau, and J. Rona. 2004. Plasma membrane depolarization induced by abscisic acid in Arabidopsis suspension cells involves reduction of proton pumping in addition to anion channel activation, which are both $\mathrm{Ca}^{2+}$ dependent. Plant Physiol. 135:231-243.

Burbidge, A., T.M. Grieve, A. Jackson, A. Thompson, D.R. McCarty, and I.B. Taylor. 1999. Characterization of the ABA-deficient tomato mutant notabilis and its relationship with maize Vp14. Plant J. 17:427-431.

Campos-Rivero, G., P. Osorio-Montalvo, R. Sanchez-Borges, R. Us-Camas, F. Duarte-Ake, and C. De-la-Pena. 2017. Plant hormone signaling in flowering: an epigenetic point of view. J. Plant Physiol. 214:16-27.

Chan, K.X., M. Wirtz, S.Y. Phua, G.M. Estavillo, and B.J. Pogson. 2013. Balancing metabolites in drought: the sulfur assimilation conundrum. Trends Plant Sci. 18:18-29. 
Chang, X., L. Donnelly, D. Sun, J. Rao, M.S. Reid, and C.-Z. Jiang. 2014. A petunia homeodomain-leucine zipper protein, PhHD-Zip, plays an important role in flower senescence. PloS One 9:e88320.

Cheng, M., P. Liao, W. Kuo, and T. Lin. 2013. The Arabidopsis ETHYLENE RESPONSE FACTOR1 regulates abiotic stress-responsive gene expression by binding to different cisacting elements in response to different stress signals. Plant Physiol. 162:1566-1582.

Conesa, A. and S. Götz. 2008. Blast2GO: a comprehensive suite for functional analysis in plant genomics. Int. J. Plant Genomics 2008.

Conner, A.J., N.W. Albert, and S.C. Deroles, 2009. Transformation and regeneration of Petunia, p. 395-409, Petunia. Springer.

Consortium, T.G. 2012. The tomato genome sequence provides insights into fleshy fruit evolution. Tomato Genome Consortium.

Couto, N., J. Wood, and J. Barber. 2016. The role of glutathione reductase and related enzymes on cellular redox homoeostasis network. Free Radical Bio. Med. 95:27-42.

Das, K. and A. Roychoudhury. 2014. Reactive oxygen species (ROS) and response of antioxidants as ROS-scavengers during environmental stress in plants. Front. Environ. Sci. 2:53.

David, R.H.A., S.A. Ceasar, K. Thirugnanasambantham, and S. Ignacimuthu. 2016. Genetic engineering of crop plants for drought tolerance: role of transcription factors. South Indian J. Biol. Sci. 2:272-286.

Davies, W.J., G. Kudoyarova, and W. Hartung. 2005. Long-distance ABA signaling and its relation to other signaling pathways in the detection of soil drying and the mediation of the plant's response to drought. J. Plant Growth Regul. 24:285.

Debbarma, J., Y.N. Sarki, B. Saikia, H.P.D. Boruah, D.L. Singha, and C. Chikkaputtaiah. 2019. Ethylene Response Factor (ERF) family proteins in abiotic stresses and CRISPR-Cas9 genome editing of ERFs for multiple abiotic stress tolerance in crop plants: a review. Mol. Biotechnol. 61:153-172.

del Amor, F.M., P. Cuadra-Crespo, D.J. Walker, J.M. Cámara, and R. Madrid. 2010. Effect of foliar application of antitranspirant on photosynthesis and water relations of pepper plants under different levels of $\mathrm{CO}_{2}$ and water stress. J. Plant Physiol. 167:1232-1238. 
Desikan, R., K. Last, R. Harrett-Williams, C. Tagliavia, K. Harter, R. Hooley, J.T. Hancock, and S.J. Neill. 2006. Ethylene-induced stomatal closure in Arabidopsis occurs via AtrbohFmediated hydrogen peroxide synthesis. Plant J. 47:907-916.

Dubois, M., L. Van den Broeck, and D. Inzé. 2018. The pivotal role of ethylene in plant growth. Trends Plant Sci. 23:311-323.

Dudhate, A., H. Shinde, D. Tsugama, S. Liu, and T. Takano. 2018. Transcriptomic analysis reveals the differentially expressed genes and pathways involved in drought tolerance in pearl millet [Pennisetum glaucum (L.) R. Br]. PloS One 13:e0195908.

Dunn, B.L., J.C. Cole, and M.E. Payton. 2012. Use of antitranspirants to reduce water stress on herbaceous and woody ornamentals. J. Environ. Hort 30:137-145.

Englert, J.M., K. Warren, L.H. Fuchigami, and T.H. Chen. 1993. Antidesiccant compounds improve the survival of bare-root deciduous nursery trees. J. Amer. Soc. Hort. Sci. 118:228-235.

Eom, S., S. Baek, J. Kim, and T. Hyun. 2018. Transcriptome analysis in Chinese cabbage (Brassica rapa ssp. pekinensis) provides the role of glucosinolate metabolism in response to drought stress. Molecules 23:1186.

Fahad, S., A.A. Bajwa, U. Nazir, S.A. Anjum, A. Farooq, A. Zohaib, S. Sadia, W. Nasim, S. Adkins, and S. Saud. 2017. Crop production under drought and heat stress: plant responses and management options. Front. Plant. Sci. 8:1147.

Fang, Y. and L. Xiong. 2015. General mechanisms of drought response and their application in drought resistance improvement in plants. Cellular Mol. Life Sci. 72:673-689.

Farooq, M., A. Wahid, N. Kobayashi, D. Fujita, and S. Basra, 2009. Plant drought stress: effects, mechanisms and management, p. 153-188. In: Lichtfouse, E., Navarrete, M., Debaeke, P., Véronique, S., and Alberola, C. (eds.), Sustainable Agriculture. Springer.

Fazal, H., A. Muhammad, A. Sartaj, S. Muhammad, U. Rizwan, and A.U. Jan. 2014. Comparative effect of polyethylene glycol and mannitol induced drought on growth (in vitro) of canola (Brassica napus), cauliflower (Brassica oleracea) and tomato (Lycopersicon esculentum) seedlings. Int. J. Biosci. 4:34-41.

Fernando, V.D. and D.F. Schroeder, 2016. Role of ABA in Arabidopsis salt, drought, and desiccation tolerance, p. 507-524, Abiotic and biotic stress in plants-recent advances and future perspectives. InTech. 
Ferrante, A., A. Trivellini, D. Scuderi, D. Romano, and P. Vernieri. 2015. Post-production physiology and handling of ornamental potted plants. Postharvest Biol. Technol. 100:99108.

Fujita, Y., M. Fujita, K. Shinozaki, and K. Yamaguchi-Shinozaki. 2011. ABA-mediated transcriptional regulation in response to osmotic stress in plants. J. Plant Res. 124:509525.

Geilfus, C.M., A. Mithöfer, J. Ludwig-Müller, C. Zörb, and K.H. Muehling. 2015. Chlorideinducible transient apoplastic alkalinizations induce stomata closure by controlling abscisic acid distribution between leaf apoplast and guard cells in salt-stressed Vicia faba. New Phytologist 208:803-816.

Gerats, T. and M. Vandenbussche. 2005. A model system for comparative research: Petunia. Trends Plant Sci. 10:251-256.

Golldack, D., I. Lüking, and O. Yang. 2011. Plant tolerance to drought and salinity: stress regulating transcription factors and their functional significance in the cellular transcriptional network. Plant Cell Rpt. 30:1383-1391.

Goreta, S., D.I. Leskovar, and J.L. Jifon. 2007. Gas exchange, water status, and growth of pepper seedlings exposed to transient water deficit stress are differentially altered by antitranspirants. J. Am. Soc. Hortic. Sci. 132:603-610.

Gupta, S. and A.M. Rashotte. 2014. Expression patterns and regulation of SlCRF3 and SlCRF5 in response to cytokinin and abiotic stresses in tomato (Solanum lycopersicum). J. Plant Physiol. 171:349-358.

Gutterson, N. and T.L. Reuber. 2004. Regulation of disease resistance pathways by AP2/ERF transcription factors. Curr. Opin. Plant Biol. 7:465-471.

Haas, B.J., A. Papanicolaou, M. Yassour, M. Grabherr, P.D. Blood, J. Bowden, M.B. Couger, D. Eccles, B. Li, and M. Lieber. 2013. De novo transcript sequence reconstruction from RNA-seq using the Trinity platform for reference generation and analysis. Nat. Protoc. $8: 1494$.

Hadiarto, T. and L.P. Tran. 2011. Progress studies of drought-responsive genes in rice. Plant Cell Rpt. 30:297-310.

Hannon, G.J. 2002. RNA interference. Nature 418:244. 
Harris, J.R. and N.L. Bassuk. 1995. Effects of defoliation and antitranspirant treatment on transplant response of scarlett oak, green ash and Turkish hazelnut. J. Arboricult. 21:3333.

Hauser, F., Z. Li, R. Waadt, and J.I. Schroeder. 2017. SnapShot: abscisic acid signaling. Cell 171:1708.

Haver, D.L., U.K. Schuch, and C.J. Lovatt. 2002. Exposure of petunia seedlings to ethylene decreased apical dominance by reducing the ratio of auxin to cytokinin. J. Plant Growth Regul. 21:459-468.

Healy, W. 2009. Piles of money. GrowerTalks Mag. 72:42-46.

Hopper, D.W., R. Ghan, K.A. Schlauch, and G.R. Cramer. 2016. Transcriptomic network analyses of leaf dehydration responses identify highly connected ABA and ethylene signaling hubs in three grapevine species differing in drought tolerance. BMC Plant Biol. $16: 118$.

Hossain, M.A., Z.-G. Li, T.S. Hoque, D.J. Burritt, M. Fujita, and S. Munné-Bosch. 2017. Heat or cold priming-induced cross-tolerance to abiotic stresses in plants: key regulators and possible mechanisms. Protoplasma 255:1-14.

Hummel, R.L. 1990. Water relations of container-grown woody and herbaceous plants following antitranspirant sprays. HortScience 25:772-775.

Iqbal, N., N.A. Khan, A. Ferrante, A. Trivellini, A. Francini, and M. Khan. 2017. Ethylene role in plant growth, development and senescence: interaction with other phytohormones. Front. Plant. Sci. 8:475.

Iriti, M., V. Picchi, M. Rossoni, S. Gomarasca, N. Ludwig, M. Gargano, and F. Faoro. 2009. Chitosan antitranspirant activity is due to abscisic acid-dependent stomatal closure. Environ. Exp. Bot. 66:493-500.

Iuchi, S., M. Kobayashi, T. Taji, M. Naramoto, M. Seki, T. Kato, S. Tabata, Y. Kakubari, K. Yamaguchi-Shinozaki, and K. Shinozaki. 2001. Regulation of drought tolerance by gene manipulation of 9-cis-epoxycarotenoid dioxygenase, a key enzyme in abscisic acid biosynthesis in Arabidopsis. Plant J. 27:325-333.

Jaleel, C.A., P. Manivannan, B. Sankar, A. Kishorekumar, R. Gopi, R. Somasundaram, and R. Panneerselvam. 2007. Water deficit stress mitigation by calcium chloride in 
Catharanthus roseus: effects on oxidative stress, proline metabolism and indole alkaloid accumulation. Colloids Surf, B Biointerfaces 60:110-116.

Janiak, A., M. Kwasniewski, M. Sowa, K. Gajek, K. Żmuda, J. Kościelniak, and I. Szarejko. 2018. No time to waste: transcriptome study reveals that drought tolerance in barley may be attributed to stressed-like expression patterns that exist before the occurrence of stress. Front. Plant. Sci. 8:2212.

Jisha, V., L. Dampanaboina, J. Vadassery, A. Mithöfer, S. Kappara, and R. Ramanan. 2015. Overexpression of an AP2/ERF type transcription factor OsEREBP1 confers biotic and abiotic stress tolerance in rice. PloS One 10:e0127831.

Johnson, S.M., F. Lim, A. Finkler, H. Fromm, A.R. Slabas, and M.R. Knight. 2014. Transcriptomic analysis of Sorghum bicolor responding to combined heat and drought stress. BMC Genomics 15:456.

Joshi, R., S.H. Wani, B. Singh, A. Bohra, Z.A. Dar, A.A. Lone, A. Pareek, and S.L. SinglaPareek. 2016. Transcription factors and plants response to drought stress: current understanding and future directions. Front. Plant. Sci. 7:1029.

Kanehisa, M. and S. Goto. 2000. KEGG: Kyoto Encyclopedia of Genes and Genomes. Nucleic Acids Res. 28:27-30.

Kazan, K. 2015. Diverse roles of jasmonates and ethylene in abiotic stress tolerance. Trends Plant Sci. 20:219-229.

Kim, J. and M.W. van Iersel. 2011. Abscisic acid drenches can reduce water use and extend shelf life of Salvia splendens. Sci. Hort. 127:420-423.

Kinoshita, T., M. Nishimura, and K. Shimazaki. 1995. Cytosolic concentration of $\mathrm{Ca}^{2+}$ regulates the plasma membrane $\mathrm{H}^{+}$-ATPase in guard cells of fava bean. Plant Cell 7:1333-1342.

Kuromori, T., J. Mizoi, T. Umezawa, K. Yamaguchi-Shinozaki, and K. Shinozaki, 2014. Drought stress signaling network, p. 383-409. In: Howell, S. H. (ed.), Molecular Biology. Springer.

Lamaoui, M., M. Jemo, R. Datla, and F. Bekkaoui. 2018. Heat and drought stresses in crops and approaches for their mitigation. Front. Chem. 6:26.

Langmead, B. and S.L. Salzberg. 2012. Fast gapped-read alignment with Bowtie 2. Nat. Methods $9: 357$. 
Li, B. and C.N. Dewey. 2011. RSEM: accurate transcript quantification from RNA-Seq data with or without a reference genome. BMC Bioinformatics 12:323.

Li, B., L. Ning, J. Zhang, M. Bao, and W. Zhang. 2015. Transcriptional profiling of Petunia seedlings reveals candidate regulators of the cold stress response. Front. Plant. Sci. 6:118.

Li, B., M. Tester, and M. Gilliham. 2017a. Chloride on the move. Trends Plant Sci. 22:236-248.

Li, K., X. Xu, and X. Huang. 2016. Identification of differentially expressed genes related to dehydration resistance in a highly drought-tolerant pear, Pyrus betulaefolia, as through RNA-Seq. PloS One 11:e0149352.

Li, P., W. Cao, H. Fang, S. Xu, S. Yin, Y. Zhang, D. Lin, J. Wang, Y. Chen, C. Xu, and Z. Yang. 2017b. Transcriptomic profiling of the maize (Zea mays L.) leaf response to abiotic stresses at the seedling stage. Front. Plant. Sci. 8:290.

Li, S., X. Han, L. Yang, X. Deng, H. Wu, M. Zhang, Y. Liu, S. Zhang, and J. Xu. 2018a. Mitogen-activated protein kinases and calcium-dependent protein kinases are involved in wounding-induced ethylene biosynthesis in Arabidopsis thaliana. Plant Cell Environ. 41:134-147.

Li, X. and F. Liu, 2016. Drought stress memory and drought stress tolerance in plants: biochemical and molecular basis, p. 17-44, Drought Stress Tolerance in Plants, Vol 1. Springer.

Li, Z., X.F. Tan, K. Lu, Z.M. Liu, and L.L. Wu. 2017c. The effect of $\mathrm{CaCl}_{2}$ on calcium content, photosynthesis, and chlorophyll fluorescence of tung tree seedlings under drought conditions. J. Photosyn. 55:553-560.

Li, Z., Y. Tian, J. Xu, X. Fu, J. Gao, B. Wang, H. Han, L. Wang, R. Peng, and Q. Yao. 2018b. A tomato ERF transcription factor, SlERF84, confers enhanced tolerance to drought and salt stress but negatively regulates immunity against Pseudomonas syringae pv. tomato DC3000. Plant Physiol. Biochem. 132:683-695.

Liu, S., J. Jin, J. Ma, M. Yao, C. Ma, C. Li, Z. Ding, and L. Chen. 2016. Transcriptomic analysis of tea plant responding to drought stress and recovery. PloS One 11:e0147306.

Ma, L., L. Hu, J. Fan, E. Amombo, A. Khaldun, Y. Zheng, and L. Chen. 2017. Cotton GhERF38 gene is involved in plant response to salt/drought and ABA. Ecotoxicology 26:841-854.

Ma, Y., X. Yu, Z. Yu, F. Sun, X. Li, and X. Li. 2018. RNA-Seq of Agropyron mongolicum Keng in response to drought stress. BMC Plant Biol. 64:3-15. 
Maathuis, F.J.M. 2013. Sodium in plants: perception, signalling, and regulation of sodium fluxes. J. Exp. Bot. 65:849-858.

Mallona, I., S. Lischewski, J. Weiss, B. Hause, and M. Egea-Cortines. 2010. Validation of reference genes for quantitative real-time PCR during leaf and flower development in Petunia hybrida. BMC Plant Biol. 10:4.

Mashaki, K.M., V. Garg, A.A.N. Ghomi, H. Kudapa, A. Chitikineni, K.Z. Nezhad, A. Yamchi, H. Soltanloo, R.K. Varshney, and M. Thudi. 2018. RNA-Seq analysis revealed genes associated with drought stress response in kabuli chickpea (Cicer arietinum L.). PloS One 13:e0199774.

Millar, A.A., J.V. Jacobsen, J.J. Ross, C.A. Helliwell, A.T. Poole, G. Scofield, J.B. Reid, and F. Gubler. 2006. Seed dormancy and ABA metabolism in Arabidopsis and barley: the role of ABA 8'-hydroxylase. Plant J. 45:942-954.

Mizoi, J., K. Shinozaki, and K. Yamaguchi-Shinozaki. 2012. AP2/ERF family transcription factors in plant abiotic stress responses. Biochim. Biophys. Acta. Gene Regul. Mech. 1819:86-96.

Müller, D. and O. Leyser. 2011. Auxin, cytokinin and the control of shoot branching. Ann. Bot. 107:1203-1212.

Müller, M. and S. Munné-Bosch. 2015. Ethylene response factors: a key regulatory hub in hormone and stress signaling. Plant Physiol. 169:32-41.

Müller, M., S. Seifert, T. Lübbe, C. Leuschner, and R. Finkeldey. 2017. De novo transcriptome assembly and analysis of differential gene expression in response to drought in European beech. PloS One 12:e0184167.

Naeem, M., M.S. Naeem, R. Ahmad, and R. Ahmad. 2017. Foliar-applied calcium induces drought stress tolerance in maize by manipulating osmolyte accumulation and antioxidative responses. Pak. J. Bot. 49:427-434.

Napoli, C., C. Lemieux, and R. Jorgensen. 1990. Introduction of a chimeric chalcone synthase gene into petunia results in reversible co-suppression of homologous genes in trans. Plant Cell 2:279-289.

Nayyar, H. 2003. Accumulation of osmolytes and osmotic adjustment in water-stressed wheat (Triticum aestivum) and maize (Zea mays) as affected by calcium and its antagonists. Environ. Exp. Bot. 50:253-264. 
Nemali, K.S. and M.W. van Iersel. 2006. An automated system for controlling drought stress and irrigation in potted plants. Sci. Hort. 110:292-297.

Nie, J., C. Wen, L. Xi, S. Lv, Q. Zhao, Y. Kou, N. Ma, L. Zhao, and X. Zhou. 2018. The AP2/ERF transcription factor CmERF053 of chrysanthemum positively regulates shoot branching, lateral root, and drought tolerance. Plant Cell Rpt. 37:1049-1060.

Niu, B., L. Fu, W. Li, Y. Gao, and Y. Huang. 2010. CD-HIT Suite: a web server for clustering and comparing biological sequences. Bioinformatics 26:680-682.

Noctor, G., G. Queval, A. Mhamdi, S. Chaouch, and C. Foyer. 2011. Glutathione. Arabidopsis Book 9:e0142.

Obidiegwu, J.E., G.J. Bryan, H.G. Jones, and A. Prashar. 2015. Coping with drought: stress and adaptive responses in potato and perspectives for improvement. Front. Plant. Sci. 6:542.

Odlum, K.D. and S.J. Colombo. 1987. The effect of three film-forming antitranspirants on moisture stress of outplanted black spruce seedlings. Tree Planters' Notes 38:23-26.

Oh, S.J., S.I. Song, Y.S. Kim, H.J. Jang, S.Y. Kim, M. Kim, Y.K. Kim, B.H. Nahm, and J.K. Kim. 2005. Arabidopsis CBF3/DREB1A and ABF3 in transgenic rice increased tolerance to abiotic stress without stunting growth. Plant Physiol. 138:341-351.

Övernäs, E., 2010. Characterisation of members of the HD-Zip I and DREB/ERF transcription factor families and their functions in plant stress responses. Acta Universitatis Upsaliensis.

Palliotti, A., S. Poni, J. Berrios, and F. Bernizzoni. 2010. Vine performance and grape composition as affected by early-season source limitation induced with anti-transpirants in two red Vitis vinifera L. cultivars. Aust, J. Grape Wine Res, 16:426-433.

Park, S., S.A. Mills, Y. Moon, and N.L. Waterland. 2016. Evaluation of antitranspirants for enhancing temporary water stress tolerance in bedding plants. HortTechnology 26:444452.

Pathan, A., M. Kimberley, W. Forster, M. Haslett, and K. Steele. 2009. Fractal characterisation of plant canopies and application in spray retention modelling for arable crops and weeds. Weed Res. 49:346-353.

Qin, X. and J.A. Zeevaart. 1999. The 9-cis-epoxycarotenoid cleavage reaction is the key regulatory step of abscisic acid biosynthesis in water-stressed bean. PNAS 96:1535415361. 
Quan, R., S. Hu, Z. Zhang, H. Zhang, Z. Zhang, and R. Huang. 2010. Overexpression of an ERF transcription factor TSRF1 improves rice drought tolerance. Plant Biotechnol. J. 8:476488.

Rabara, R.C., P. Tripathi, R.N. Reese, D.L. Rushton, D. Alexander, M.P. Timko, Q.J. Shen, and P. Rushton. 2015. Tobacco drought stress responses reveal new targets for Solanaceae crop improvement. BMC Genomics 16:484.

Raines, T., C. Shanks, C.Y. Cheng, D. McPherson, C.T. Argueso, H.J. Kim, J.M. FrancoZorrilla, I. López-Vidriero, R. Solano, and R. Vaňková. 2016. The cytokinin response factors modulate root and shoot growth and promote leaf senescence in Arabidopsis. Plant J. 85:134-147.

Ranty, B., D. Aldon, V. Cotelle, J. Galaud, P. Thuleau, and C. Mazars. 2016. Calcium sensors as key hubs in plant responses to biotic and abiotic stresses. Front. Plant. Sci. 7:327.

Raz, V. and R. Fluhr. 1992. Calcium requirement for ethylene-dependent responses. Plant Cell 4:1123-1130.

Robinson, M.D., D.J. McCarthy, and G.K. Smyth. 2010. edgeR: a Bioconductor package for differential expression analysis of digital gene expression data. Bioinformatics 26:139140.

Roelfsema, M.R.G., R. Hedrich, and D. Geiger. 2012. Anion channels: master switches of stress responses. Trends Plant Sci. 17:221-229.

Rong, W., L. Qi, A. Wang, X. Ye, L. Du, H. Liang, Z. Xin, and Z. Zhang. 2014. The ERF transcription factor TaERF3 promotes tolerance to salt and drought stresses in wheat. Plant Biotechnol. J. 12:468-479.

Sah, S.K., K.R. Reddy, and J. Li. 2016. Abscisic acid and abiotic stress tolerance in crop plants. Front. Plant. Sci. 7:571.

Saito, S., N. Hirai, C. Matsumoto, H. Ohigashi, D. Ohta, K. Sakata, and M. Mizutani. 2004. Arabidopsis CYP707As encode (+)-abscisic acid 8'-hydroxylase, a key enzyme in the oxidative catabolism of abscisic acid. Plant Physiol. 134:1439-1449.

Sassi, S., S. Aydi, K. Hessini, E.M. Gonzalez, C. Arrese-Igor, and C. Abdelly. 2010. Long-term mannitol-induced osmotic stress leads to stomatal closure, carbohydrate accumulation and changes in leaf elasticity in Phaselous vulgaris leaves. African J. of Biotechno. 9:6061-6069. 
Savvides, A., S. Ali, M. Tester, and V. Fotopoulos. 2016. Chemical priming of plants against multiple abiotic stresses: mission possible? Trends Plant Sci. 21:329-340.

Serna, L. and C. Fenoll. 2001. Ethylene induces stomata differentiation in Arabidopsis. Int. J. Dev. Biol. 40:S123-S124.

Sharma, N., D.R. Waterer, and S.R. Abrams. 2006. Evaluation of abscisic acid analogs as holding agents for bedding plant seedlings. HortTechnology 16:71-77.

Shi, C., H. Yang, C. Wei, O. Yu, Z. Zhang, C. Jiang, J. Sun, Y. Li, Q. Chen, and T. Xia. 2011. Deep sequencing of the Camellia sinensis transcriptome revealed candidate genes for major metabolic pathways of tea-specific compounds. BMC Genomics 12:131.

Shinohara, T. and D.I. Leskovar. 2014. Effects of ABA, antitranspirants, heat and drought stress on plant growth, physiology and water status of rtichoke transplants. Sci. Hort. 165:225234.

Shoresh, M., M. Spivak, and N. Bernstein. 2011. Involvement of calcium-mediated effects on ROS metabolism in the regulation of growth improvement under salinity. Free Radical Bio. Med. 51:1221-1234.

Singh, R., P. Parihar, S. Singh, R.K. Mishra, V.P. Singh, and S.M. Prasad. 2017. Reactive oxygen species signaling and stomatal movement: current updates and future perspectives. Redox Biol. 11:213-218.

Slama, I., T. Ghnaya, K. Hessini, D. Messedi, A. Savouré, and C. Abdelly. 2007. Comparative study of the effects of mannitol and PEG osmotic stress on growth and solute accumulation in Sesuvium portulacastrum. Environ. Exp. Bot. 61:10-17.

Steinberg, S.L., M.J. McFarland, and J.W. Worthington. 1990. Antitranspirant reduces water use by peach trees following harvest. J. Amer. Soc. Hort. Sci. 115:20-24.

Sugano, S., H. Kaminaka, Z. Rybka, R. Catala, J. Salinas, K. Matsui, M. Ohme-Takagi, and H. Takatsuji. 2003. Stress-responsive zinc finger gene ZPT2-3 plays a role in drought tolerance in petunia. Plant J. 36:830-841.

Suwa, R., K. Jayachandran, N.T. Nguyen, A. Boulenouar, K. Fujita, and H. Saneoka. 2008. Barium toxicity effects in soybean plants. Arch. Environ. Contam. Toxicol. 55:397-403.

Taiz, L. and E. Zeiger. 2010. Plant phyiology. 5th ed. Sinauer, Sunderland, MA. 
Takeuchi, J., M. Okamoto, R. Mega, Y. Kanno, T. Ohnishi, M. Seo, and Y. Todoroki. 2016. Abscinazole-E3M, a practical inhibitor of abscisic acid 8'-hydroxylase for improving drought tolerance. Sci. Rpt. 6:37060.

Tan, W., Q. wei Meng, M. Brestic, K. Olsovska, and X. Yang. 2011. Photosynthesis is improved by exogenous calcium in heat-stressed tobacco plants. J. Plant Physiol. 168:2063-2071.

Tanaka, Y., T. Sano, M. Tamaoki, N. Nakajima, N. Kondo, and S. Hasezawa. 2005. Ethylene inhibits abscisic acid-induced stomatal closure in Arabidopsis. Plant Physiol. 138:23372343.

Tanaka, Y., T. Sano, M. Tamaoki, N. Nakajima, N. Kondo, and S. Hasezawa. 2006. Cytokinin and auxin inhibit abscisic acid-induced stomatal closure by enhancing ethylene production in Arabidopsis. J. Exp. Bot. 57:2259-2266.

Tao, J., H. Chen, B. Ma, W. Zhang, S. Chen, and J. Zhang. 2015. The role of ethylene in plants under salinity stress. Front. Plant. Sci. 6:1059.

Tian, X., Y. Long, J. Wang, J. Zhang, Y. Wang, W. Li, Y. Peng, Q. Yuan, and X. Pei. 2015. De novo transcriptome assembly of common wild rice (Oryza rufipogon Griff.) and discovery of drought-response genes in root tissue based on transcriptomic data. PloS One 10:e0131455.

Tuteja, N. and S. Mahajan. 2007. Calcium signaling network in plants: an overview. Plant Signal. Behav. 2:79-85.

Tuteja, N. and S.K. Sopory. 2008. Chemical signaling under abiotic stress environment in plants. Plant Signal. Behav. 3:525-536.

Upadhyaya, H., S.K. Panda, and B.K. Dutta. 2011. $\mathrm{CaCl}_{2}$ improves post-drought recovery potential in Camellia sinensis (L) O. Kuntze. Plant Cell Rpt. 30:495-503.

USDA. 2014. Floriculture crops 2013 summary. U.S. Dept. Agr., Washington, D.C.

USDA. 2019. Floriculture crops 2018 summary. U.S. Dept. Agr., Washington, D.C.

Verma, V., P. Ravindran, and P.P. Kumar. 2016. Plant hormone-mediated regulation of stress responses. BMC Plant Biol. 16:86.

Villarino, G.H., A. Bombarely, J.J. Giovannoni, M.J. Scanlon, and N.S. Mattson. 2014. Transcriptomic analysis of Petunia hybrida in response to salt stress using high throughput RNA sequencing. PloS One 9:e94651. 
Vishwakarma, K., N. Upadhyay, N. Kumar, G. Yadav, J. Singh, R.K. Mishra, V. Kumar, R. Verma, R.G. Upadhyay, M. Pandey, and S. Sharma. 2017. Abscisic acid signaling and abiotic stress tolerance in plants: a review on current knowledge and future prospects. Front. Plant. Sci. 8:161.

Wan, L., J. Zhang, H. Zhang, Z. Zhang, R. Quan, S. Zhou, and R. Huang. 2011. Transcriptional activation of OsDERF1 in OsERF3 and OsAP2-39 negatively modulates ethylene synthesis and drought tolerance in rice. PloS One 6:e25216.

Wang, F., H. Yamamoto, and Y. Ibaraki. 2008. Measuring leaf necrosis and chlorosis of bamboo induced by typhoon 0613 with RGB image analysis. J. For. Res. 19:225-230.

Wang, K.L.C., H. Li, and J.R. Ecker. 2002. Ethylene biosynthesis and signaling networks. Plant Cell 14:S131-S151.

Wang, M., B. Jiang, Q. Peng, W. Liu, X. He, Z. Liang, and Y. Lin. 2018. Transcriptome analyses in different cucumber cultivars provide novel insights into drought stress responses. Int. J. Mol. Sci. 19:7.

Wang, W., B. Vinocur, and A. Altman. 2003. Plant responses to drought, salinity and extreme temperatures: towards genetic engineering for stress tolerance. Planta 218:1-14.

Wang, X., H. Han, J. Yan, F. Chen, and W. Wei. 2015. A new AP2/ERF transcription factor from the oil plant Jatropha curcas confers salt and drought tolerance to transgenic tobacco. Appl.Biochem. Biotechnol. 176:582-597.

Waterland, N.L., C.A. Campbell, J.J. Finer, and M.L. Jones. 2010a. Abscisic acid application enhances drought stress tolerance in bedding plants. HortScience 45:409-413.

Waterland, N.L., J.J. Finer, and M.L. Jones. 2010b. Abscisic acid applications decrease stomatal conductance and delay wilting in drought-stressed chrysanthemums. HortTechnology 20:896-901.

Waterland, N.L., J.J. Finer, and M.L. Jones. 2010c. Benzyladenine and gibberellic acid application prevents abscisic acid-induced leaf chlorosis in pansy and viola. HortScience 45:925-933.

Watkins, J.M., P.J. Hechler, and G.K. Muday. 2014. Ethylene-induced flavonol accumulation in guard cells suppresses reactive oxygen species and moderates stomatal aperture. Plant Physiol. 164:1707-1717. 
Weaver, G.M. and M.W. van Iersel. 2014. Antitranspirational efficacy and longevity of abscisic acid and a synthetic abscisic acid analog in pansies (Viola $\times$ wittrockiana). HortScience 49:779-784.

Wei, S., W. Hu, X. Deng, Y. Zhang, X. Liu, X. Zhao, Q. Luo, Z. Jin, Y. Li, and S. Zhou. 2014. A rice calcium-dependent protein kinase OsCPK9 positively regulates drought stress tolerance and spikelet fertility. BMC Plant Biol. 14:1.

Wilson, R.C. and J.A. Doudna. 2013. Molecular mechanisms of RNA interference. Annu. Rev. Biophys. 42:217-239.

Wuriyanghan, H., B. Zhang, W. Cao, B. Ma, G. Lei, Y. Liu, W. Wei, H. Wu, L. Chen, and H. Chen. 2009. The ethylene receptor ETR2 delays floral transition and affects starch accumulation in rice. Plant Cell 21:1473-1494.

Xie, Z., T.M. Nolan, H. Jiang, and Y. Yin. 2019. AP2/ERF transcription factor regulatory networks in hormone and abiotic stress responses in Arabidopsis. Front. Plant. Sci. 10:228.

Xiong, L. and J.K. Zhu. 2003. Regulation of abscisic acid biosynthesis. Plant Physiol. 133:29-36.

Xu, C., X. Li, and L. Zhang. 2013a. The effect of calcium chloride on growth, photosynthesis, and antioxidant responses of Zoysia japonica under drought conditions. PloS One 8:e68214.

$\mathrm{Xu}$, J. and S. Zhang. 2014. Regulation of ethylene biosynthesis and signaling by protein kinases and phosphatases. Mol. Plant 7:939-942.

Xu, Y., S. Gao, Y. Yang, M. Huang, L. Cheng, Q. Wei, Z. Fei, J. Gao, and B. Hong. 2013 b. Transcriptome sequencing and whole genome expression profiling of chrysanthemum under dehydration stress. BMC Genomics 14:662.

Yang, D., J. Jin, L. Kong, Y. Meng, G. Gao, J. Luo, and F. Tian. 2016. PlantTFDB 4.0: toward a central hub for transcription factors and regulatory interactions in plants. Nucleic Acids Res. 45:D1040-D1045.

Yates, S.A., M.T. Swain, M.J. Hegarty, I. Chernukin, M. Lowe, G.G. Allison, T. Ruttink, M.T. Abberton, G. Jenkins, and L. Skøt. 2014. De novo assembly of red clover transcriptome based on RNA-Seq data provides insight into drought response, gene discovery and marker identification. BMC Genomics 15:1. 
Yu, J., L. Niu, J. Yu, W. Liao, J. Xie, J. Lv, Z. Feng, L. Hu, and M.M. Dawuda. 2019. The involvement of ethylene in calcium-induced adventitious root formation in cucumber under salt stress. Int. J. Mol. Sci. 20:1047.

Yue, C. and B.K. Behe. 2008. Estimating US consumers' choice of floral retail outlets. HortScience 43:764-769.

Zemlyanskaya, E.V., N.A. Omelyanchuk, A.A. Ermakov, and V.V. Mironova. 2017. Mechanisms regulating ethylene signal transduction in plants. Russ. J. Genet. Appl. Res. $7: 335-344$.

Zhang, J., W. Jia, J. Yang, and A.M. Ismail. 2006. Role of ABA in integrating plant responses to drought and salt stresses. Field Crops Res. 97:111-119.

Zheng, H., J. Ye, J. Chen, J. Wang, J. Wang, L. Bolund, L. Fang, R. Li, S. Li, Y. Zhang, and Z. Zhang. 2006. WEGO: a web tool for plotting GO annotations. Nucleic Acids Res. 34:W293-W297.

Zhou, A., H. Ma, E. Liu, T. Jiang, S. Feng, S. Gong, and J. Wang. 2017. Transcriptome sequencing of Dianthus spiculifolius and analysis of the genes involved in responses to combined cold and drought stress. Int. J. Mol. Sci. 18:849.

Zhou, Z., H. Ma, K. Lin, Y. Zhao, Y. Chen, Z. Xiong, L. Wang, and B. Tian. 2015. RNA-seq reveals complicated transcriptomic responses to drought stress in a nonmodel tropic plant, Bombax ceiba L. Evolutionary Bioinformatics 11:27-37. 